


\title{
Three-Dimensional \\ Ultrasound Imaging of the Prostate
}

\author{
by \\ Shidong Tong \\ Department of Medical Biophysics
}

\begin{abstract}
Submitted in partial fulfillment
of the requirements for the degree of

Doctor of Philosophy
\end{abstract}

Faculty of Graduate Studies

The University of Western Ontario

London, Ontario, Canada

August 1997

C Shidong Tong 1997 
National Library

of Canada

Acquisitions and Bibliographic Services

395 Wellington Street Ottawa ON K1A ON4 Canada
Bibliothèque nationale du Canada

Acquisitions et services bibliographiques

395, rue Wellington

Ottawa ON K1A ON4

Canada
The author has granted a nonexclusive licence allowing the National Library of Canada to reproduce, loan, distribute or sell copies of this thesis in microform, paper or electronic formats.

The author retains ownership of the copyright in this thesis. Neither the thesis nor substantial extracts from it may be printed or otherwise reproduced without the author's permission.
L'auteur a accordé une licence non exclusive permettant à la Bibliothèque nationale du Canada de reproduire, prêter, distribuer ou vendre des copies de cette thèse sous la forme de microfiche/film, de reproduction sur papier ou sur format électronique.

L'auteur conserve la propriété du droit d'auteur qui protège cette thèse. Ni la thèse ni des extraits substantiels de celle-ci ne doivent être imprimés ou autrement reproduits sans son autorisation. 


\section{Abstract}

Prostate cancer is the most commonly diagnosed cancer in men in North America. Since its introduction in 1971, transrectal ultrasound (TRUS) has evolved into one of the primary tools for the evaluation of prostate disease. However, with conventional TRUS, diagnosticians must mentally integrate a series of 2D images in order to develop an impression of the 3D anatomy, and the accurate and precise estimation of prostate volume is difficult. Moreover, an examination takes 10-20 minutes, often resulting in patient discomfort.

We propose using 3D TRUS to overcome these problems. In this thesis, we describe a 3D ultrasound imaging system, analyze the distortion in its images due to miscalibration, and study its performance in estimating prostate volume.

Our system consists of three elements: (i) a conventional ultrasound machine and TRUS probe; (ii) a custom-built assembly for rotating the probe under microcomputer control; and, (iii) a microcomputer with an 8-bit video frame grabber. A typical scan of $2002 \mathrm{D}$ B-mode images takes 13 seconds, so that the entire examination takes under 5 minutes. These images can then be rapidly reconstructed into a 3D image using an algorithm we describe, and interactively displayed using standard software. Also, we describe procedures for accurately calibrating the reconstruction parameters, so that distortions in the shape, length, area, and volume of the 3D image of an object are all less than $1 \%$.

We also show that manual planimetry of 3D TRUS images can be used to estimate prostate volumes in vitro with $2.6 \%$ accuracy and $2.5 \%$ precision; and in vivo with $5.1 \%$ intra-observer variability and $11.4 \%$ inter-observer variability. By comparison, with the height-width-length method (Volume $=\pi / 6 \mathrm{HWL}$ ) with conventional 20 TRUS images, in vivo estimates had $11.5 \%$ intra-observer variability and $13.5 \%$ inter-observer variability.

Thus, 3D TRUS overcomes the limitations of 2D TRUS, and has great potential as a tool for the diagnosis, study, and management of prostate disease.

Keywords: Three-dimensional, 3-D, 3D, ultrasound, sonography, transrectal, TRUS, imaging, distortion, prostate, volume, accuracy, precision, variability 


\section{Acknowledgements}

I would like to thank my supervisor, Dr. Aaron Fenster, for his guidance, advice, and encouragement throughout my tenure as a graduate student. Also, I would like to thank him and the other members of my advisory committee, Drs. Ting Lee and Brian Rutt, for their advice and comments on this thesis.

I wish to thank all the people at the Imaging Research Laboratories and Life Imaging Systems, Inc., who supported my work over the years. In particular, I would like to thank Dr. Neale Cardinal for his assistance in analyzing my data, editing my papers and my thesis, and helping me to improve my English. I am also grateful to Dr. Ken Chu, Jennifer Nolan, and Katherine Draper for their valuable comments and suggestions on my thesis. In addition, I appreciate the lively conversations, and the good advice on a broad range of topics, which made it fun to work in "the Dungeon".

Thanks also go to Drs. Ross Mitchell and Paul Picot, who generously provided me with access to their computer software libraries; to Drs. Dan Rickey, Ian Cunningham, David Holdsworth, Maria Drangova, and Michael Eliasziw, who generously shared their expertise with me; and, to my fellow graduate students, who made studying here enjoyable.

I also wish to express my gratitude to Drs. Dónal Downey and Richard Rankin for their clinical advice; to Rick Gerson, Siyuan Lei, Abir Zahalka, and Drs. Dónal Downey, Tracy Elliot, Marielle Fortier, and Ray McLoughlin for their participation in the variability study; to John Miller and Susan Thielen for their technical support; and to the Medical Research Council of Canada and the Ontario Graduate Scholarship Program for their financial support.

Finally, I would like to thank my wife Siyuan Lei for her understanding and encouragement, for putting up with my long hours in the lab, and for her help with my work, and to thank the rest of my family as well for their continued support. 


\section{Table of Contents}

Certificate of Examination................................................................................... ii

Abstract........................................................................................... ii

Acknowledgments........................................................................................... iv

Table of Contents .......................................................................................

List of Figures ....................................................................................... ix

List of Tables ................................................................................................

List of Abbreviations ......................................................................................iii

1. INTRODUCTION .................................................................................................

1.1 Prostate Cancer ....................................................................................

1.2 Screening for Prostate Cancer .....................................................................

1.2.1 Digital rectal examination (DRE) ............................................... 4

1.2.2 The prostate specific antigen (PSA) test...................................... 4

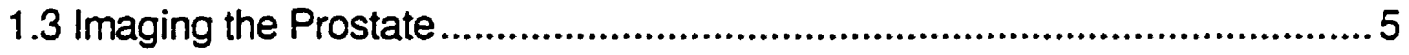

1.3.1 Computed tomography (CT) ..................................................

1.3.2 Magnetic resonance imaging (MRI) ............................................

1.3.3 Transrectal ultrasound (TRUS) ................................................ 6

1.4 Limitations of Conventional TRUS ........................................................

1.5 Staging and Prostate Volume Estimation................................................. 8

1.6 Hypothesis and Research Goals............................................................ 8

1.7 Three-Dimensional (3D) Ultrasound Imaging...........................................

1.7.1 Historical background ...................................................................

1.7.2 Prostate imaging...................................................................... 10

1.7 .3 Image acquisition.......................................................................11

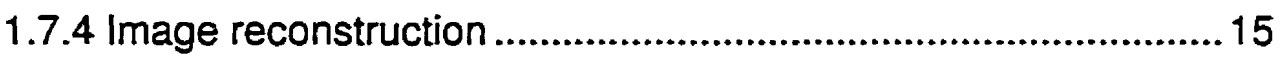

1.7 .5 Image display ………............................................................. 16

1.8 Thesis Outline

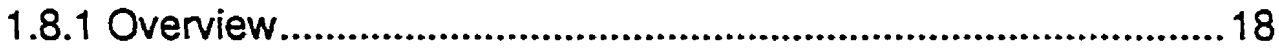

1.8.2 Development of the 3D ultrasound imaging system ..................19

1.8.3 Accuracy of prostate volume measurement in vitro ....................20 


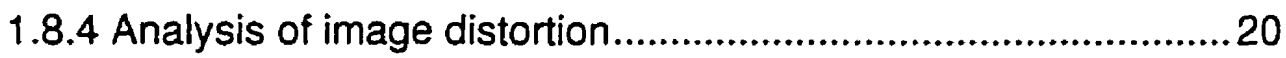

1.8.5 Intra- and inter-observer variability.........................................21

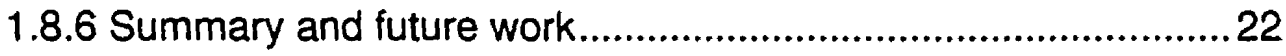

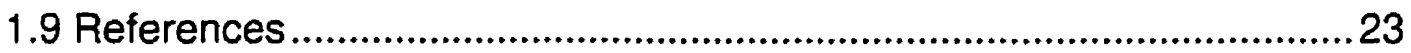

2. DEVELOPMENT OF THE 30 ULTRASOUND IMAGING SYSTEM...............28

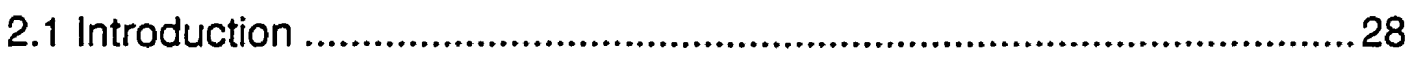

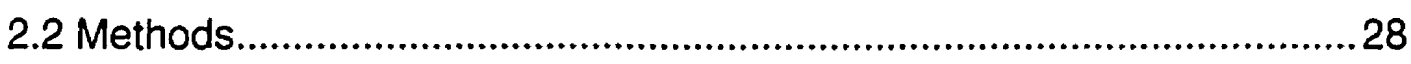

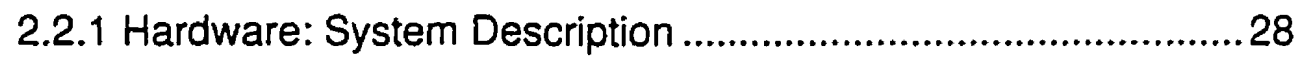

2.2.2 Software: 3D Image Reconstruction ..........................................30

2.2.3 Evaluation of System Performance .......................................... 33

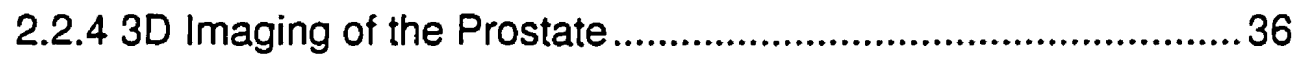

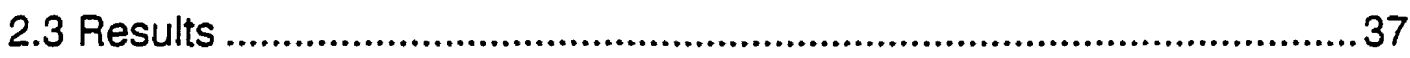

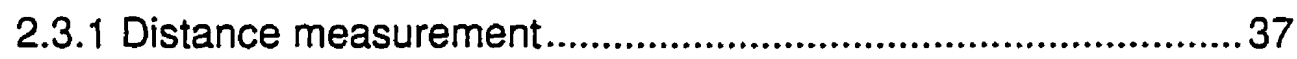

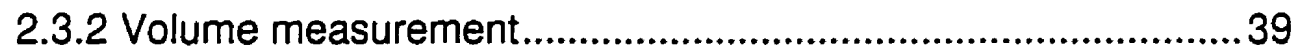

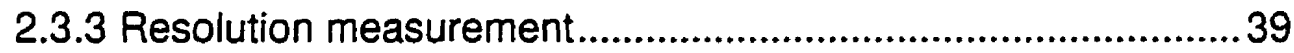

2.3.4 3D imaging of the prostate ......................................................42

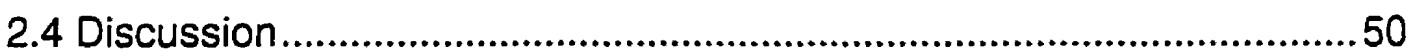

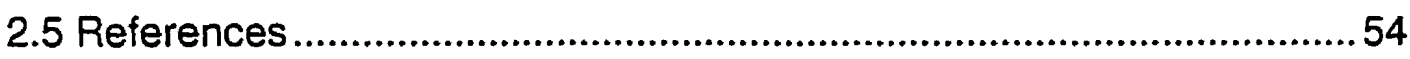

3. ACCURACY OF VOLUME MEASUREMENTS IN VITRO..........................55

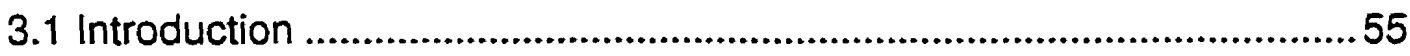

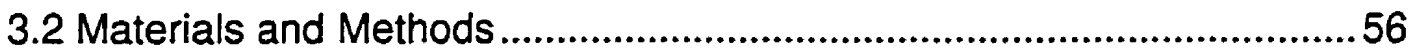

3.2.1 Cadaver specimens .................................................................56

3.2.2 3D ultrasound imaging technique ........................................56

3.2.3 Accuracy of volume measurements .........................................57

3.2.4 Preparation of pathology slides ...........................................58

3.2.5 Comparison of 3D US slices and pathology slides...................59

3.2.6 Precision of area and volume measurements ...........................59

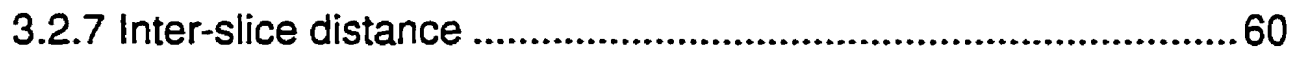

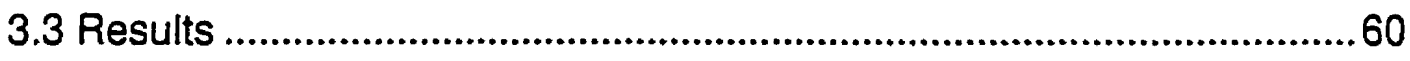

3.3.1 Accuracy of volume measurements....................................60 60 
3.3.2 Comparison of 3D US slices vs pathology slides 60

3.3.3 Precision of area and volume measurements .........................63

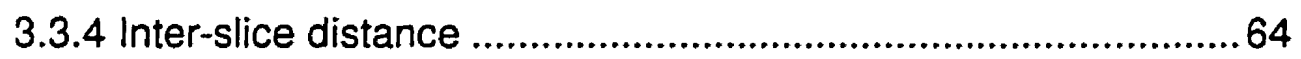

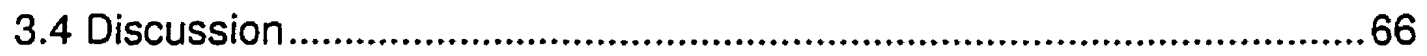

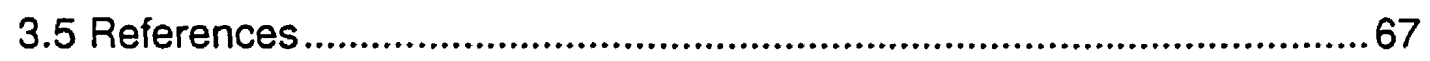

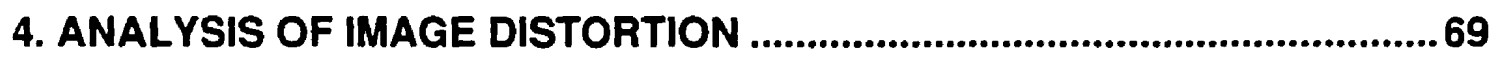

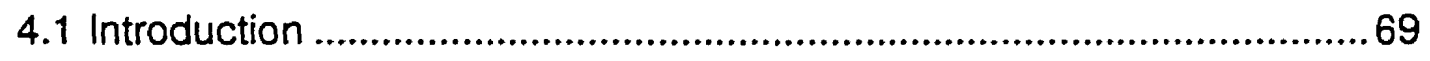

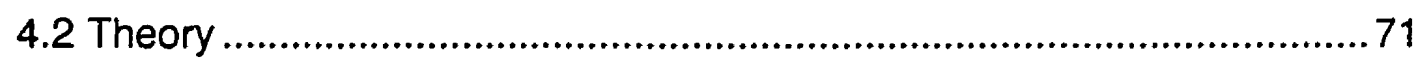

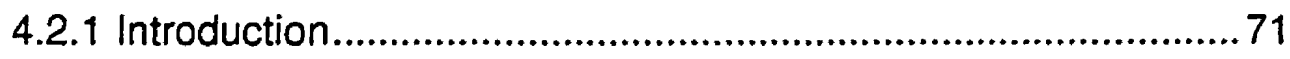

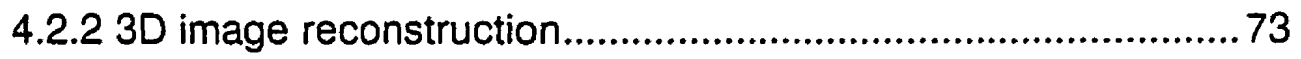

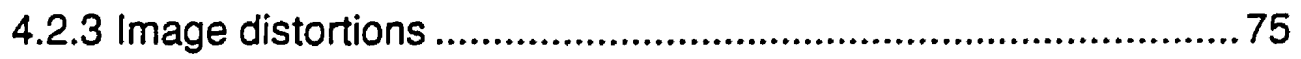

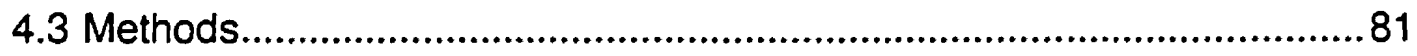

4.3.1 Computer simulation of errors ................................................ 81

4.3.2 Experimental measurement of errors...................................... 84

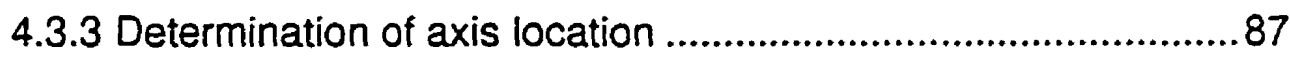

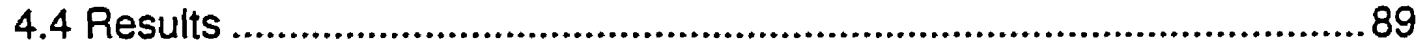

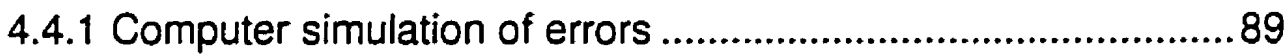

4.4.2 Experimental measurement of errors........................................93

4.4.3 Determination of axis location ............................................ 100

4.5 Discussion and Conclusions .............................................................. 102

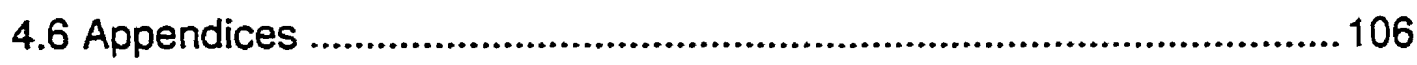

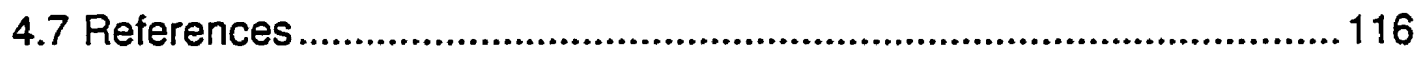

5. INTRA- AND INTER-OBSERVER VARIABILITY ..................................118

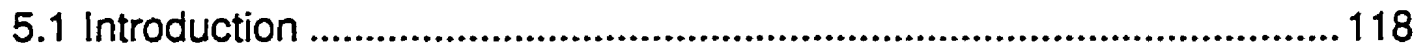

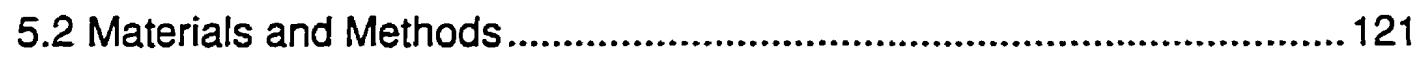

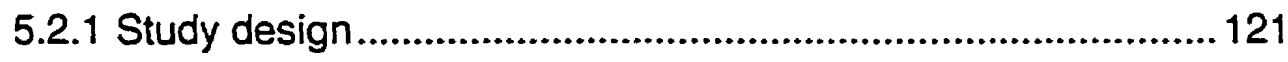

5.2.2 Standard deviations of volume measurements ............................... 122

5.2.3 Systematic and random observer errors.............................. 123

5.2.4 Analysis of Variance ................................................................ 124

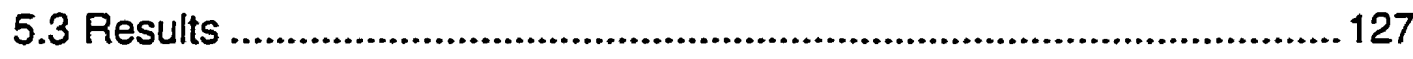


5.3.1 Standard deviations of volume measurements 127

5.3.2 Systematic and random observer errors.............................. 129

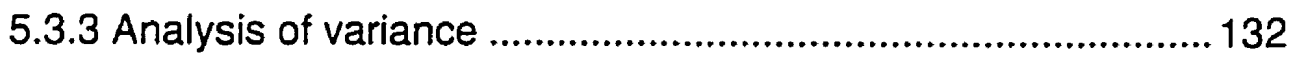

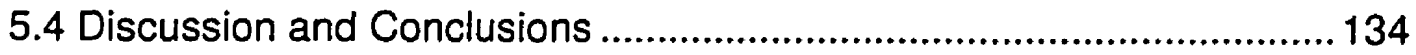

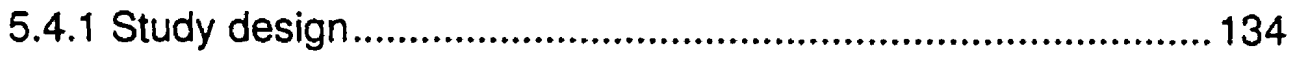

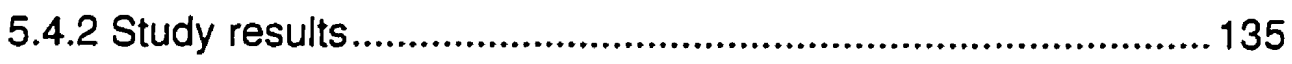

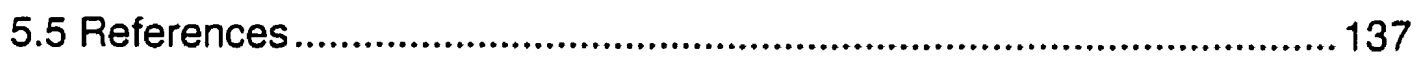

6. SUMMARY, DISCUSSION, AND FUTURE WORK ....................................140

6.1 Summary and Discussion................................................................... 140

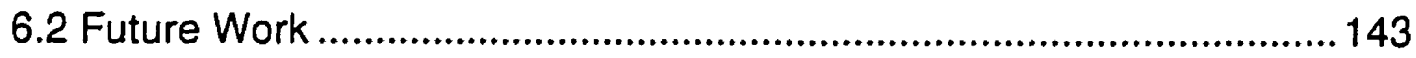

6.2.1 Improvement of the system................................................... 143

6.2.2 Effect of 2D image slice thickness....................................... 144

6.2.3 Automated image segmentation .......................................... 144

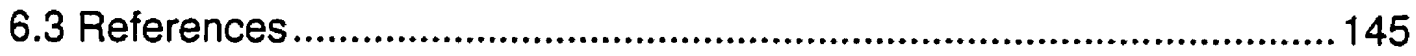

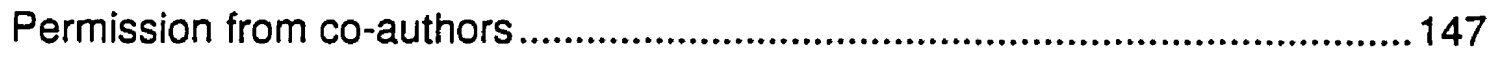

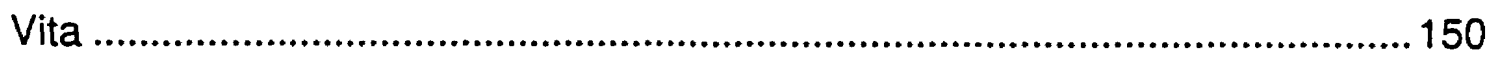




\section{List of Figures}

Figure 1.1 The annual number of new cases and deaths due to prostate cancer in the United States, in thousands, from 1980 to 1995

Figure 1.2 The annual number of new cases and deaths due to prostate cancer in the United States, as a percentage of all cancers, from 1980 to 1995 ... 2

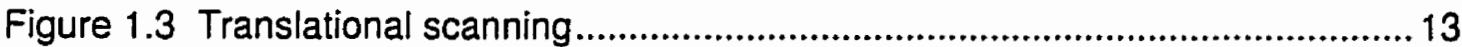

Figure 1.4 Rotational scanning with an end-firing probe.......................................... 14

Figure 1.5 Rotational scanning with a side-firing probe.............................................. 15

Figure 1.6 Multiplanar texture mapped image of a 3D TRUS image of a

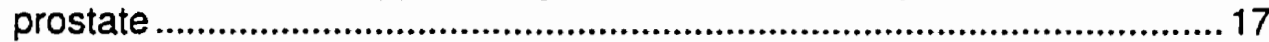

Figure 1.7 Minimum-intensity projection of a prostate and its attached seminal vesicles, shown for two different azimuthal angles ..................................... 18

Figure 2.1 Photograph of a transrectal ultrasound (TRUS) probe, mounted on a computer-controlled motorized rotational mechanism

Figure 2.2 Schematic diagram outlining the process of 3D image acquisition and reconstruction.

Figure 2.3 Schematic diagram illustrating the geometry used to describe the reconstruction algorithm

Figure 2.4 A photograph of the test phantom...

Figure 2.5 Full-width at half-maximum (FWHM) of the string cross-sectional images in the lateral and axial directions for string layers at various distances $y$ from the probe.

Figure 2.6 The increase in the full-width at half-maximum (FWHM) of the string cross-sectional images caused by the $3 \mathrm{D}$ reconstruction algorithm

Figure 2.7 3D images of a prostate are "sliced" to reveal the tumor and the peri-prostatic fat.

Figure 2.8 By "slicing" into the prostate, the tumor can be seen in both transaxial and sagittal planes. 44

Figure 2.9 The coronal plane of the prostate 45

Figure 2.10 The hematoxylin- and eosin-stained slide of the tumor as shown in Figure 2.7, following radical prostatectomy.

Figure 2.11 Four views of a translucency-rendered prostate image, at four different angles, derived from the same image data used for Figure 2.7 .

Figure 2.12 Cross-sectional slices from a 3D image of the prostate reconstructed using nearest-neighbor interpolation.

Figure 2.13 Cross-sectional slices from a 3D image of the prostate reconstructed using linear interpolation. 
Figure 2.14 Cross-sectional slices from a 3D image of the prostate reconstructed using bilinear interpolation.

Figure 3.1 The prostate volume measured from the three-dimensional (3D) ultrasound image compared with the volume measured by water displacement

Figure 3.2 Prostate area obtained from digitized pathology slides compared to the area obtained from corresponding views from the 3D ultrasound image slices

Figure 3.3 The prostate volume measured by outlining a 3D image slice-byslice for inter-slice distance ranging from 1 to $15 \mathrm{~mm}$. The slicing of the 3D image was carried out in three viewing planes: transaxial, sagittal, and coronal planes.

Figure 4.1 A schematic diagram illustrating the geometry used for 3D scanning........71

Figure 4.2 Schematic diagram illustrating the variables used to analyze the image distortion of a straight line object 74

Figure 4.3 Graphs of the function $F(\alpha, \beta, \theta)$ appearing in Eqs. (4.1) and (4.2) .77

Figure 4.4 Graphs of the function $G(\alpha, \beta, \theta)$ appearing in Eqs. (4.1) and (4.3). 78

Figure 4.5 Schematic diagram illustrating an $x-y$ cross-section of the $3 \mathrm{D}$ image of a white cube set in a gray background.

Figure 4.6 Schematic cross-section of the calibration phantom

Figure 4.7 An $x-y$ cross-section of the 3D ultrasound image of the wire phantom

Figure 4.8 Central portions of $x-y$ cross-sections of 30 ultrasound images of the wire phantom, similar to Figure 4.7 , but reconstructed using a range of parameter values $R_{0}{ }^{\prime}$

Figure 4.9 Central portions of $x-y$ cross-sections of 3D ultrasound images of the wire phantom, similar to Figure 4.7, but reconstructed using a range of parameter values $\Theta^{\prime}$

Figure 4.10. Graph of the maximum relative perpendicular distortion $h / \ell$ in the reconstructed image of a straight line perpendicular to and bisected by the radial line $\theta=0$

Figure 4.11 Graph of the relative distortion $\Delta \ell / \ell$ in the length of the reconstructed image of a straight line perpendicular to and bisected by the radial line $\theta=0$

Figure 4.12 Graph of the relative distortion $\Delta A / A=\left(A^{\prime}-A\right) / A$ in the area of the parallelogram containing the central nine centroids of Figure 4.7 ...... 99

Figure 4.13 Plot of axis location vs reciprocal pixel size 102

Figure 5.1 Manually drawn outline of the prostate in a sample cross-sectional 2D image "sliced" from a 3D ultrasound image of the prostate. 120

Figure 5.2 Measured prostate volume vs prostate number for the HWL and 3D 
US methods

Figure 5.3 Relative standard deviation vs prostate number for the HWL and 3D US methods

Figure 5.4 Relative standard deviation vs prostate volume for the HWL and $3 D$ US methods

Figure 5.5 Systematic observer error vs observer number, for the HWL and $3 D$ US methods

Figure 5.6 Random observer error vs observer number, as a percentage of prostate volume. 


\section{List of Tables}

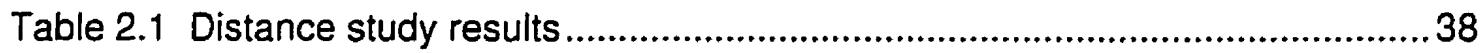

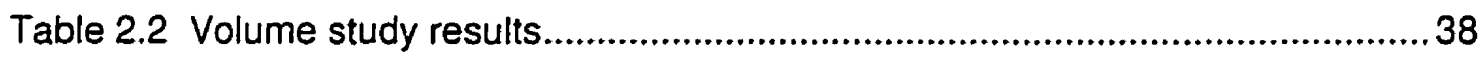

Table 2.3 CPU times for reconstructing a 3D image ...................................................50

Table 3.1 Inverse prediction of the true volumes and their $95 \%$ confidence interval for the 3D US measured volumes...................................................63

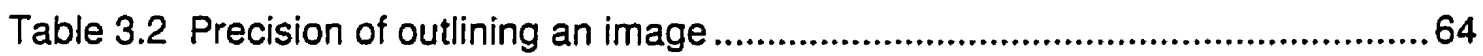

Table 4.1. Comparison of measured vs theoretical relative errors $\Delta \ell / \ell$. in the measured length $\ell^{\prime}=\ell+\Delta \ell$ of line $L$ of Figure 4.5 , as a function of $\Delta R$

Table 4.2. Comparison of measured vs theoretical relative errors $\Delta \ell . / \ell$. in the measured length $\ell^{\prime}=\rho+\Delta \ell$ of line $L$ of Figure 4.5, as a function of $\Delta \Theta$.

Table 4.3. Comparison of measured vs theoretical relative errors $\triangle A V A$ in the measured area $A^{\prime}=A+\triangle A$ of the square of Figure 4.5, as a function of $\Delta R$.

Table 4.4. Comparison of measured vs theoretical relative errors $\triangle A / A$ in the measured area $A^{\prime}=A+\Delta A$ of the square in Figure 4.5, as a function of $\Delta \Theta$

Table 4.5. Experimentally measured location of the probe rotation axis for different fields-of-view

Table 5.1 Data layout for repeated measurements of prostate volumes 123

Table 5.2 Analysis of variance (ANOVA) table for repeated measurement experiments used to study intra- and inter-observer reliability and variability simultaneously.

Table 5.3 Mean and standard deviation of systematic observer error. 130

Table 5.4 Mean and standard deviation of random observer error. 131

Table 5.5 The intra- and inter-observer reliability coefficients $p_{\text {intra }}$ and $p_{\text {inter }}$ from an analysis of variance (ANOVA) of prostate volume measurements made by the HWL and 3D US methods.

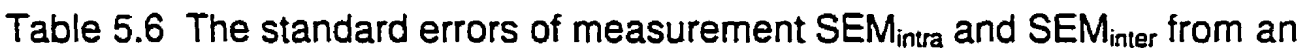
analysis of variance (ANOVA) of prostate volume measurements made by the HWL and 3D US methods

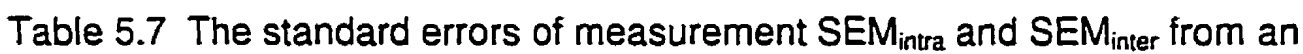
analysis of variance of normalized prostate volume measurements by the HWL and 3D US methods 


\section{List of Abbreviations}

$\begin{array}{ll}\text { 2D } & \text { two-dimemsional } \\ \text { 3D } & \text { three-dimemsional } \\ \text { ANOVA } & \text { analysis of variance } \\ \text { BPH } & \text { benign prostatic hypertrophy } \\ \text { CT } & \text { computed tomography } \\ \text { DRE } & \text { digital rectal examination } \\ \text { FOV } & \text { field of view } \\ \text { FWHM } & \text { full-width at half-maximum } \\ \text { HWL } & \text { height-width-length (method) } \\ \text { MR } & \text { magnetic resonance } \\ \text { MRI } & \text { magnetic resonance imaging } \\ \text { PSA } & \text { prostate specific antigen } \\ \text { RAM } & \text { random access memory } \\ \text { ROI } & \text { region of interest } \\ \text { SD } & \text { standard deviation } \\ \text { SEM } & \text { standard error of measurement } \\ \text { TRUS } & \text { transrectal ultrasound imaging } \\ \text { US } & \text { ultrasound, ultrasound imaging }\end{array}$




\section{INTRODUCTION}

\subsection{Prostate Cancer}

The prostate gland is a small organ in men, similar in shape and size to a large walnut, which lies below the bladder, and surrounds the bladder neck and urethra. This gland is an important part of the male sexual anatomy. It provides an alkaline viscid fluid which constitutes the major component of seminal fluid. The size of a normal prostate is about $4 \mathrm{~cm}$ in diameter, and $20 \mathrm{~g}$ in weight.

Because of the small size of this organ, many men are unaware that it even exists until prostate disease begins to affect them late in life. The most common and important diseases of the prostate are benign prostatic hypertrophy and prostate cancer.

Benign prostatic hypertrophy (BPH) is an enlargement of the prostate gland which occurs in almost all older men. More than $50 \%$ of men over the age of 50 have some degree of $\mathrm{BPH}$, and its prevalence increases with age. Ultimately, $80 \%$ to $90 \%$ of all adult males can expect to be affected with this condition (Rifkin, 1988). The clinical implications of BPH can be both immense and debilitating, since an enlarged prostate obstructs the flow of urine through the urethra. Typical symptoms of BPH include a slow, intermittent urinary stream, hesitancy, and incomplete bladder emptying.

Prostate cancer is the most commonly diagnosed cancer in men. It has the second highest mortality rate, after lung cancer, among all cancers in men in North America (Wingo et al. 1995). For every year since 1980, the number of new cases and number of deaths due to prostate cancer in the United States is shown in thousands in Figure 1.1, and as a percentage of all cancers in Figure 1.2. From these figures, it is clear that the annual incidence rate of prostate cancer has increased in the United States every year since 1980, and dramatically so in the last decade. In 1995, there were 244,000 new cases and more than 40,000 deaths due to prostate cancer in the United States. 


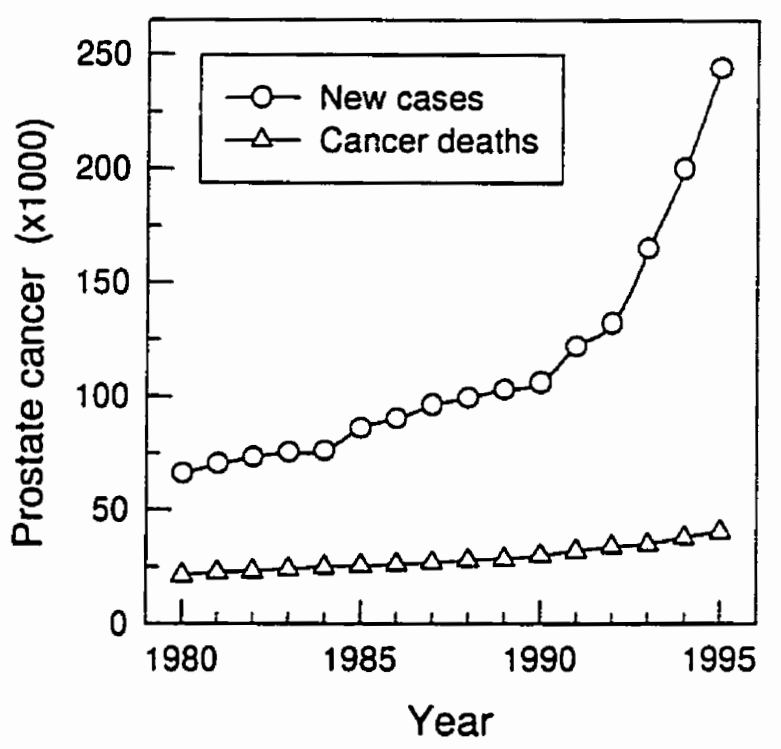

Figure 1.1 The annual number of new cases and deaths due to prostate cancer in the United States, in thousands, from 1980 to 1995 (Cancer statistics. Cancer J. Clin.; 1980-1995).

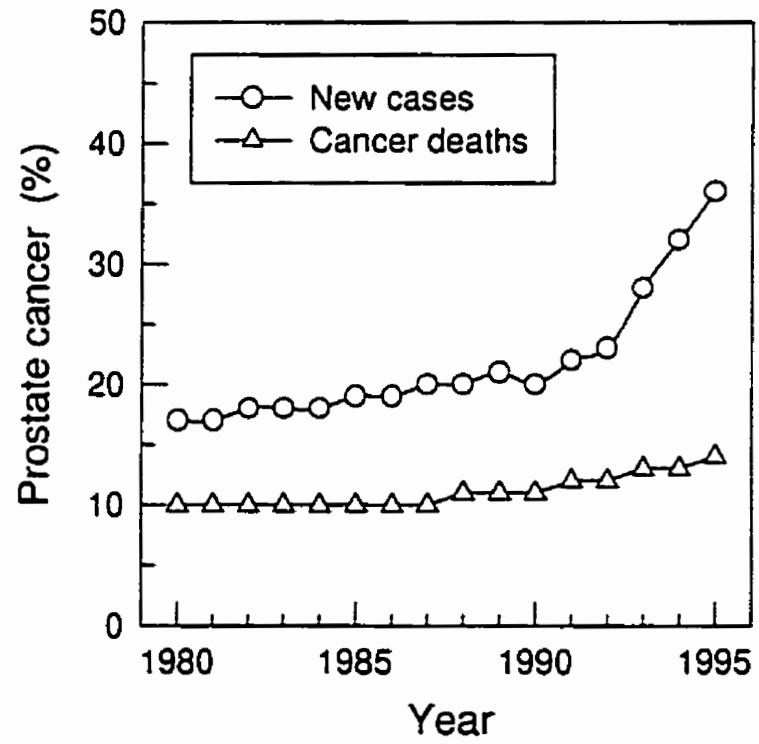

Figure 1.2 The annual number of new cases and deaths due to prostate cancer in the United States, as a percentage of all cancers, from 1980 to 1995 (Cancer statistics. Cancer J. Clin.; 1980-1995). 
In Canada, the situation is similar. According to Canadian Cancer Statistics 1996, prostate cancer is the most commonly diagnosed cancer in Canadian men, and it ranks second only to lung cancer as a cause of death due to cancer (National Cancer Institute of Canada 1996). In 1996, there were 18,200 new cases and 4,200 deaths due to prostate cancer in Canada. By comparison, in 1987, there were 8,200 new cases and 2,800 deaths. Thus, the number of new cases has increased by $122 \%$, and the number of deaths increased by $50 \%$, in only nine years. It is generally accepted that much of the increase in new cases is due to recent advances in early detection.

It is estimated that some $30 \%$ of men over the age of 50 have prostate cancer (McNeal 1983). However, many of them remain asymptomatic until extensive local growth or metastasis of the tumour has occurred. Once the tumour is no longer encapsulated within the prostate, there is a dramatic increase in the risk of metastasis, and a corresponding decrease in the prospect of a cure. When diagnosed at an early stage, prostate cancer is curable; nevertheless, even in the later stages, treatment can still be effective. Thus, the early diagnosis and accurate staging of prostate cancer are of primary importance.

\subsection{Screening for Prostate Cancer}

Because of its inaccessible location, clinical assessment of the prostate gland is difficult. Traditionally, this has been done by physical examination, prostatic fluid inspection, biopsy, or surgery. However, during the last two decades, several imaging modalities have been developed to aid in the diagnosis of prostate disease, particularly computed tomography (CT), magnetic resonance imaging (MRI), and transrectal ultrasound (TRUS).

At present, the most commonly used screening techniques for prostate cancer are the digital rectal examination (DRE) and the prostate specific antigen (PSA) test. 


\subsubsection{Digital rectal examination (DRE)}

DRE is the most commonly employed screening technique for prostate cancer. In this examination, the clinician assesses the size and the shape of the prostate with his or her fingers. It is a simple, inexpensive, and readily available procedure. Unfortunately, it also tends to detect only those tumours which have attained a size associated with aggressive biological activity. Furthermore, it evaluates only the posterior portion of the prostate. As a result, $40-50 \%$ of prostate tumours escape detection by this method, because they are not palpable (Lee et al. 1989).

\subsubsection{The prostate specific antigen (PSA) test}

Human prostate specific antigen (PSA) was discovered in 1979, and today its role in the diagnosis and monitoring of prostate cancer is well established. A simple blood test can signal the presence of prostate cancer in a man who has no other symptoms of prostate abnormality, by measuring the level of PSA secreted by the prostate gland. The introduction of this assay has dramatically altered the way prostate cancer patients are monitored, particularly after treatment. However, the use of PSA for the early detection and staging of prostate cancer remains controversial, and forms the subject of many clinical and scientific investigations (Guinan et al. 1987; Drago et al. 1989; Benson 1994). Although a slight increase in the PSA level is observed in patients in the early stages of prostate cancer, and a large increase in the advanced stages, BPH and other prostate diseases can also increase the PSA level. As a consequence, PSA is an imperfect screening test for prostate cancer, and it cannot be relied upon alone for the accurate diagnosis and staging of prostate cancer.

In an effort to improve the clinical utility of the PSA test, many investigators have attempted to correlate the PSA level with the prostate volume (PSA density). Although it has been claimed that the discriminating power of the PSA test can be improved in this way (Benson et al. 1992a, 1992b), other investigators could not confirm this finding (Brawer et al. 1993). However, it is generally believed that 
measuring the prostate and/or tumour volume is important in interpreting the PSA level. Until now, this task has been performed using TRUS, although with less accuracy than clinically desirable (Terris et al. 1992).

\subsection{Imaging the Prostate}

\subsubsection{Computed tomography (CT)}

CT is a widely used $x$-ray imaging modality, in which images are obtained tomographically, or slice-by-slice. For each slice, the attenuation of $x$-rays incident in that plane is measured for many angles of incidence, and the distribution of the $x$-ray attenuation coefficient within the slice is computed (Johns and Cunningham 1983, Cho et al. 1993). The CT image of a normal prostate is a homogeneous object with a clearly defined outline. Thus, although the prostate volume can be readily measured, the image lacks sufficient contrast to discern the internal architecture of the prostate. The sensitivity (the ability to correctly identify those people who have the disease) and specificity (the ability to distinguish those people who do not have the disease) of CT is low for extracapsular tumour extension or seminal-vesicle invasion (Kirby et al. 1996). Furthermore, it cannot reliably differentiate between BPH and prostate cancer. Consequently, the value of CT imaging in the evaluation of prostate cancer is limited, and it has therefore been superseded by MRI and TRUS.

\subsubsection{Magnetic resonance imaging (MRI)}

MRI is a relatively new and rapidly developing imaging modality, which has several advantages over CT. Unlike $C T$, it is non-invasive, since it does not use ionizing radiation for image formation. Also, it is better able to delineate the internal structure of the prostate, due to its superior image contrast. Moreover, with MRI, the prostate can be imaged in transverse, sagittal, and coronal planes; but, with $C T$, only transverse planes are available. On the other hand, MRI examinations are costly, and not readily available in Canada. 
However, although MRI has been shown to be able to detect tumours in the peripheral zone of the prostate, it cannot readily distinguish between benign and malignant growths. A large scale multi-institutional cooperative study has shown that MRI has a sensitivity of $77 \%$ and a specificity of $57 \%$. Only about $60 \%$ of lesions will be indentified (Rifkin 1990, Rifkin et al. 1990). Considering its high cost and limited diagnostic accuracy, it is clear that MRI requires some improvements before it may be effectively used for diagnosing prostate cancer.

\subsubsection{Transrectal ultrasound (TRUS)}

TRUS imaging has several advantages over CT and MRI for imaging the prostate. Unlike CT and MRI machines, ultrasound machines are portable and inexpensive to operate. Moreover, since the images are generated from ultrasonic waves, TRUS is non-invasive and image formation is fast, allowing real-time procedures such as ultrasound-guided biopsy to be performed. Also, although MRI is superior to CT for staging prostate cancer, it is not significantly superior to TRUS, a much less expensive technique (Smith and Resnick 1993). Moreover, TRUS provides reasonable resolution and soft-tissue contrast in prostate images, and has proven to be valuable in the staging, monitoring, and biopsy of prostate cancer. For these reasons, TRUS is currently the most commonly used modality for imaging the prostate.

Since its introduction in 1971 (Watanabe et al. 1971), TRUS has become a primary tool in the evaluation of the prostate disease. Early images, obtained with 3.5 $\mathrm{MHz}$ transducers, provided information about the size and shape of the prostate (King 1973). Sagittal and transverse scanners, introduced in the late 1970's and early 1980 's, clearly showed two distinct zones within the prostate gland, and the higher-frequency ( 5 and $7 \mathrm{MHz}$ ) transducers introduced in the mid 1980's resulted in improved image resolution and display of the zonal anatomy. Over the past decade, specialized ultrasound machines and transducers have been developed for diagnosing prostate disease, and, aided by recent advances in computer-assisted imaging technology, it is now possible to see very small prostate lesions with TRUS. 
As a result, the number of instruments used for prostate examinations, and the number of radiologists and urologists performing TRUS, has increased dramatically during this time. Currently, the uses of TRUS include: (i) screening, for the early detection of prostate cancer; (ii) ultrasound-guided biopsy, for the diagnosis and staging of prostate cancer; (iii) ultrasound-guided brachytherapy, for prostate cancer therapy; and, (iv) monitoring, for determining the response of prostate cancer to various treatment modalities (Lee et al. 1989). Of these, the one with the greatest clinical impact may be the first, which has increased the detection rate of prostate cancer during its early, i.e. confined and curable, stage. In one screening program, Lee et al. (1988) examined 784 men aged 60 or over with TRUS and DRE, and found that the detection rate of prostate cancer with TRUS was double that of DRE (2.6\% vs $1.3 \%)$.

However, despite its rapid proliferation, the role of TRUS in the diagnosis and staging of prostate cancer remains controversial. This is because, although a conventional TRUS examination has been shown to have significant advantages, it has also been shown to have relatively low sensitivity and specificity for the staging of prostate cancer. A multicentre trial comparing TRUS with MRI for the staging of localized prostate cancer found that TRUS had an overall staging accuracy of $58 \%$, a sensitivity of $46 \%$, and a specificity of $66 \%$, while MRI was $11 \%$ higher in each of these categories (Rifkin et al. 1990). Thus, although it is generally agreed that TRUS is an important and cost-effective technique for the diagnosis and staging of prostate cancer, it is also widely recognized that some technical improvements to TRUS are needed before its potential can be fully realized .

\subsection{Limitations of Conventional TRUS}

One of the major limitations of conventional TRUS images are their inherently two-dimensional (2D) character, since human anatomy is three-dimensional (3D). Thus, diagnosticians must mentally integrate a series of $2 D$ images in order to develop an impression of the 3D anatomy, an arduous and time-consuming process prone to operator variability. 
Also, due to the restrictions imposed by patient anatomy, only transverse and sagittal views are available with conventional 2D TRUS, and not coronal or oblique views, hindering this visualization process and hence the accurate diagnosis or staging of the patient's condition.

Moreover, since a 2D image represents a thin slice of the patient's anatomy in a particular orientation and location, it is difficult to locate the same image plane in subsequent examinations. For this reason, $2 D$ imaging modalities such as conventional TRUS are not ideal for prospective or follow-up studies, such as monitoring the effects of cancer therapy.

Furthermore, due to its duration of 10-20 minutes, a conventional TRUS examination is usually an uncomfortable procedure, particularly for elderly patients who cannot lie for long on their hips.

\subsection{Staging and Prostate Volume Estimation}

Prostate cancer is categorized into different stages, depending on the tumour size, whether it is confined, and whether it has metastasized. Differentiation of these stages therefore requires the prostate and tumour volumes to be known with high accuracy. Also, the reproducibility of prostate volume estimates is important for interpreting the PSA assay level, and any change of it in response to therapy.

Currently, quantitative estimation of the prostate or tumour volume is usually performed by measuring its height, width, and length from two orthogonal 2D TRUS images (the transverse and sagittal views) and calculating the volume of the corresponding ellipsoid. This is called the HWL method. However, this method may potentially lead to inaccurate results, particularly for tumours with irregular shapes.

\subsection{Hypothesis and Research Goals}

Our hypothesis is that using a 3D ultrasound imaging system to perform 3D TRUS may overcome the aforementioned limitations of conventional 2D TRUS, and may permit the estimation of prostate and tumour volumes with greater accuracy and consistency than afforded by the HWL method. In this way, we hope to achieve 
our overall research goal of making the diagnosis and staging of prostate cancer more accurate and less operator dependant.

We propose a computerized 3D ultrasound imaging system that can scan a patient's prostate in only a few seconds. The resulting 3D image can be later manipulated and viewed interactively on a computer, after the patient has departed, and can be used to measure prostate and tumour volumes with more accuracy and reproducibility than the HWL method. Our specific research goals were:

1. to develop a 3D ultrasound imaging system for performing 3D TRUS of the prostate, based on a conventional ultrasound machine and transducer;

2. to evaluate the imaging performance (accuracy, precision, resolution, distortion) of this system in vitro, using suitable phantoms and prostate specimens;

3. to determine theoretically, verify numerically, and measure experimentally the types of image distortion for this system and their magnitudes, and to devise methods for reducing these magnitudes to negligible levels; and,

4. to show, using images obtained clinically in vivo, that the estimation of prostate volume by the 3D ultrasound method (to be described) is statistically significantly better than by the HWL method, in terms of intra- and inter-observer variability.

Each of these, in sequence, forms the topic of a chapter in the body of this thesis. These chapters are described in detail below, in the thesis outline. However, as a prelude to this outline, we first review the state-of the-art in 3D ultrasound imaging leading up to our work.

\subsection{Three-Dimensional (3D) Ultrasound Imaging}

\subsubsection{Historical background}

Several recent articles comprehensively review 3D ultrasound imaging, including both the technical and the clinical aspects (Fenster and Downey 1996, Foster 1995, Rankin et al. 1993, Greenleaf et al. 1993, Belohlavek et al. 1993). Here, we summarize some important developments. 
Baum and Greenwood (1961) were the first to describe the concept of 3D ultrasound imaging. They created a 3D display by stacking sequential photographic plates containing ultrasound images of the orbit of the human eye. Since then, considerable advances have been made: for example, Dekker et al. (1974) were among the first to create 3D ultrasound scans of the heart, using a mechanical arm to track the transducer motion as it was scanned across the chest.

Later, Greenleaf (1982) described three different techniques for obtaining 3D ultrasound images: (i) ultrasound B-mode; (ii) ultrasound computed tomography; and, (iii) Doppler ultrasound. Briefly, his techniques may be summarized as follows:

To create a 3D B-mode image, a carotid artery specimen was scanned in vitro by moving the transducer slowly along the artery axis to obtain $75 \mathrm{~B}$-mode images, approximately $0.3 \mathrm{~mm}$ apart, which were digitized and stored on magnetic tape for subsequent analysis and 3D image display.

To create a 3D ultrasound CT image, the same technique as used for $x$-ray CT imaging was employed: Ultrasonic pulses were transmitted through an excised breast in coronal planes, approximately $1 \mathrm{~mm}$ apart, "from a plurality of directions", and the received signals were processed for arrival time and amplitude. A series of $2 D$ tomograms, showing either the distribution of acoustic speed or attenuation within each plane, were then computed via a conventional CT reconstruction algorithm.

Finally, using Doppler velocimetry to obtain a velocity profile through a carotid artery specimen in vitro, a 2D velocity distribution of flow velocity was built up by traversing the transducer across the artery, and then displayed as a $3 D$ plot of flow velocity vs $2 D$ cross-sectional position within the artery, to show the paraboloidal velocity profile characteristic of laminar flow.

\subsubsection{Prostate imaging}

A number of techniques for 3D ultrasound imaging of the prostate have been reported (Littrup 1987; Watanabe 1989; Höhne at al. 1990, Sehgal et al. 
1994). In each case, multiple $2 D$ cross-sectional images of the prostate were acquired as the transducer was scanned in some linear fashion. The prostate was then manually outlined in each recorded image, and the resulting set of outlines was used to generate an outside view of the prostate, either as a shaded polyhedron (Littrup 1987) or as a smooth surface-rendered image (Watanabe 1989; Höhne at al. 1990, Sehgal et al. 1994).

However, this type of $3 D$ image retains no information about the interior of the prostate. Also, the unfamiliarity of radiologists with surface-rendered images makes their diagnostic interpretation difficult.

\subsubsection{Image acquisition}

The process of 3D ultrasound imaging has three major stages: (1) image acquisition; (2) image processing and reconstruction; and, (3) image display. In stage 1 , multiple $2 \mathrm{D}$ images are acquired, together with information on their positions and orientations; in stage 2, this data is used to generate a 3D image of the scanned volume; and, in stage 3 , a computer is used to view this image in various ways. Here, we describe stage 1 . Stages 2 and 3 are described in subsequent sections.

In order to combine the acquired set of 2D images into a single 3D image, the relative position and orientation of each $2 \mathrm{D}$ image must be known. There are basically two ways to accomplish this: The first is to record the transducer's relative position and orientation as it is manually scanned across the volume of interest, using some sort of tracking device. This is called free-hand scanning. The second is to use some mechanical device to move the transducer in some predetermined fashion, so that the relative position and orientation of each 2D image are similarly predetermined. This is called mechanical scanning. We now consider both of these approaches in some detail.

\subsubsection{Free-hand scanning}

In free-hand scanning, the 2D images are acquired at irregular positions and 
orientations, which must be recorded via some sort of tracking device. So far, three types of devices have been developed: mechanical, acoustic, and electromagnetic.

Mechanical: This is the simplest approach. Here, the transducer is mounted on a jointed mechanical arm, and the angulations of the joints are measured by potentiometers as the arm is moved. This method has been used for imaging the human heart (Sawada 1983) and fetus (Baba et al. 1989).

Acoustic: This method of tracking is based on the acoustic range principle. Here, a triangular array of three sonic emitters is attached to the transducer, and a square array of four microphones above the patient then measures the time-of-flight for pulses from each emitter to each microphone. From this data, the transducer position and orientation may be calculated (Brinkley et al. 1982, Moritz et al. 1983). Using this system, 2D image plane separations can be measured with an accuracy of $0.4 \%$, and 3D volumes estimated with an accuracy of $1.6 \%$ (King et al. 1990, 1991).

Electromagnetic: A pulsed magnetic field position/orientation system has been recently developed (Flock-of-Birds, Ascension Technologies, Burlington VT) and used in 3D ultrasound imaging (Hodges et al. 1994; Fenster et al. 1995). A fixed transmitter, located near the patient, produces a spatially varying magnetic field, which is measured by a receiver, mounted on the transducer. The position and orientation of the receiver, relative to the transmitter, can then be calculated to typical accuracy of 0.1 inch and $0.5^{\circ}$, respectively (Bird Model 6D-BRD, Ascension Technologies).

However, it is important to note that, although free-hand scanning offers significant flexibility in acquiring the $2 D$ image data, care must be taken to ensure that the number of images is sufficient to cover the object under study, and that there are no big gaps between successive images.

\subsubsection{Mechanical scanning}

In this approach, the ultrasound transducer is mounted on some mechanical device, and its movement is generally restricted to only one degree 
of freedom, either translation along an axis, or rotation about an axis. Since the transducer motion is predetermined, it does not need to be tracked. Moreover, since the $2 \mathrm{D}$ images are regularly spaced and oriented, 3D image reconstruction can generally be performed much more efficiently than with free-hand scanning, where the 2D images are irregularly spaced and oriented. Furthermore, the relative position and orientation of each $2 D$ image is known much more precisely with mechanical scanning than with free-hand scanning, so that the final 3D image is also more precise. Here, we consider three types of mechanical scans: translation, rotation of an end-firing probe, and rotation of a side-firing probe.

Translation: Here, the transducer is held by a mechanical device, which is linearly translated (e.g. by a lead screw) so that the $2 D$ images are uniformly spaced and parallel, at a fixed angle to the direction of motion (Figure 1.3).

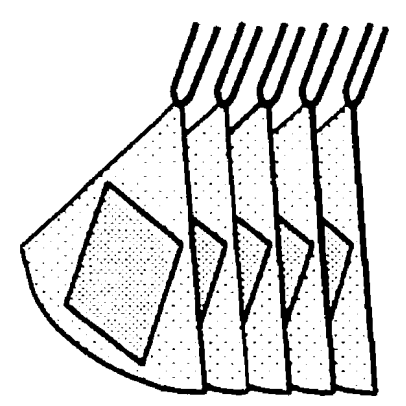

Figure 1.3: Translational scanning

When this angle is $90^{\circ}$, the $3 \mathrm{D}$ image simply consists of the stack of $2 \mathrm{D}$ images; using this approach, Halliwell et al. (1989) were able to generate 2D prostate images in sagittal, coronal, and oblique planes from a set of $2 \mathrm{D}$ images originally scanned in transverse planes, but did not generate a 3D image.

However, when the $2 \mathrm{D}$ image planes are not at $90^{\circ}$ to the scanning direction, as in a colour Doppler scan of an artery, a shear operation must be applied to the stack of $2 D$ images in order to recover the correct 3D geometry, i.e. to embed the 2D images properly in the 3D image volume (Picot et al. 1993). 
Rotation of an end-firing probe: This type of scan is illustrated in Figure 1.4. Here, the transducer is at the probe tip, and the probe is rotated through $180^{\circ}$ about its axis, which extends through the cone-shaped volume containing the acquired $2 D$ images, in order to collect sufficient data for $3 D$ image reconstruction. However, with this scan geometry, great care must be taken to align the rotation axis properly and to keep it stationary, since even a slight deviation of the axis from the $2 \mathrm{D}$ image plane, or any tilt or displacement of the axis during the scan, will cause noticeable artifacts in the reconstructed $3 D$ image. Because of this sensitivity, it is generally preferable to perform rotational scanning with a side-firing probe.

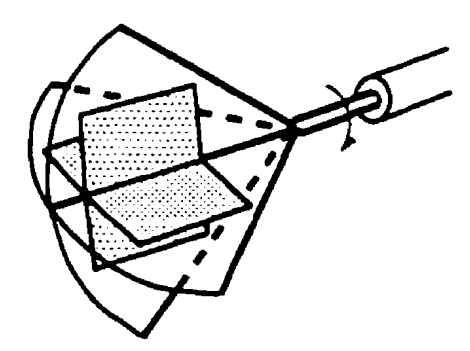

Figure 1.4: Rotational scanning with an end-firing probe.

Rotation of a side-firing probe: This type of scan is illustrated in Figure 1.5. Here, the transducer is parallel to the probe axis, and the $2 \mathrm{D}$ image planes sweep out a fan-shaped volume as the probe is rotated about its axis though an angle sufficient to cover the volume of interest, typically $80^{\circ}$ for a prostate scan. With this scan geometry, the rotation axis lies outside the scan volume. Hence, any slight tilt or displacement of the axis during the scan, relative to the orientation and location assumed by the reconstruction algorithm, will only cause slight geometric distortions to appear in the reconstructed 3D image, and no other artifacts. Because of this insensitivity, this type of scan was adopted for use with our 3D ultrasound imaging system. 


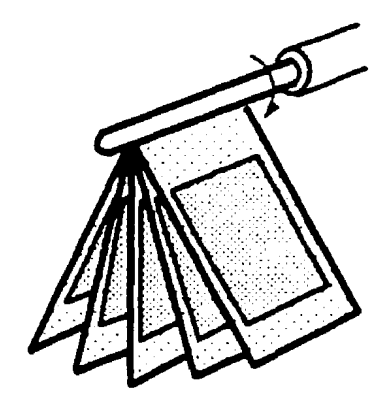

Figure 1.5: Rotational scanning with a side-firing probe.

\subsubsection{Image reconstruction}

Since 2D ultrasound images are tomographic in nature, the process of $3 D$ image reconstruction involves embedding a series of $2 \mathrm{D}$ images in their correct positions and orientations in a 3D volume, and then using suitable interpolation techniques to calculate the 3D image intensity at the nodes of a regular grid in Cartesian coordinates $(x, y, z)$ in this volume. The set of embedded 2D images, with the image intensity defined at the $(x, y, z)$ coordinates of the $2 \mathrm{D}$ image pixels, is called the source image; while the interpolated image, with the image intensity defined at the $(x, y, z)$ coordinates of the grid nodes, is called the destination image; and the process of determining the intensities (voxel values) of the destination image from those of the source image is called 3D image reconstruction. This is done in order to facilitate image display using standard image processing software.

This process may be performed in two ways: via the source-oriented method, or via the destination-oriented method. With the former method, the image intensity of each pixel in the source image is distributed among its nearest neighbours in the destination image; while, with the latter method, the intensity of each voxel in the destination image is interpolated from its nearest neighbours in the source image.

For translational scanning, both methods work well, since the 2D image pixels are uniformly spaced. However, for rotational scanning, this is not the case. 
For a rotational step size of $\Delta \theta$, the pixels at radius $r$ are spaced a distance $r \Delta \theta$ apart in the $\theta$ direction. Consequently, for the source-oriented method, destination image voxels may receive contributions from many source-image pixels when $r$ is small, and contributions from no source-image pixels when $r$ is large. While radially rescaling the destination image intensity can fix the first problem, it cannot fill in the gaps in the destination image caused by the second problem. On the other hand, with the destination-oriented method, where each voxel in the destination image is interpolated from its nearest neighbours in the source image, regardless of their distance. Therefore, the destination-oriented method of 3D image reconstruction was adopted for our system (Tong et al. 1996).

\subsubsection{Image display}

In the past decade, several standard techniques have been developed for displaying 3D images whose voxel values are given on a regular Cartesian grid. These are briefly described here.

\subsubsection{Multiplanar texture mapping}

This method of display is illustrated by Figure 1.6. Here, the 3D image is displayed as a cube (or other polyhedron), with the appropriate image texture mapped onto each face. Thus, for a 3D ultrasound image, each face displays a conventional (B-mode, power Doppler, or colour Doppler) 2D image of the anatomy for that plane, as interpolated from the 3D image data. Furthermore, this display can be viewed interactively, by moving the image planes, i.e. "slicing" into the cube; by adding new, oblique planes; or by rotating the cube to any desired orientation (Robb 1995). Moreover, since the images on each face are simply conventional $2 \mathrm{D}$ images, already familiar to radiologists, whose diagnostic interpretation is straightforward, this type of display can be viewed as an enhanced version of a conventional $2 \mathrm{D}$ image display. 


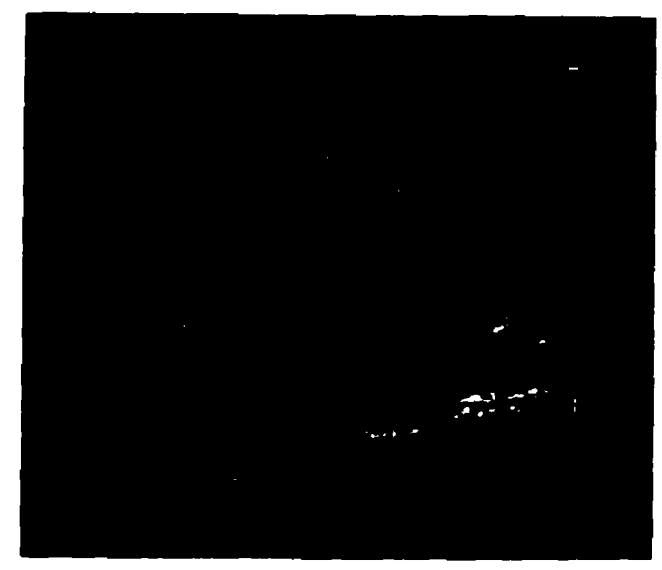

Figure 1.6: Multiplanar texture mapped image of a 3D TRUS image of a prostate with a tumour, visible as the anechoic region on the lower near edge of the cube.

\subsubsection{Surface rendering}

This technique displays a picture of a 3D surface, with visual cues such as perspective, shading, and shadowing added, to help convey a 3D impression of its shape. Preprocessing of the 3D image data is usually required before a surface-rendered image can be generated, since the image data must be segmented into different classes (e.g. tissue types) before the desired surface may be extracted for rendering. This segmentation can be accomplished by manual outlining, simple thresholding, or sophisticated image-processing algorithms. The desired surface may then be displayed as a wire-frame image, a shaded polyhedron, or a smoothly shaded surface, which can be rotated to any desired orientation (Robb 1995). Moreover, by using two copies of the image, rotated to slightly different angles, a stereo view may be generated by presenting the images separately to the viewer's left and right eyes (e.g. by using left and right circularly polarized filters and a specialized display unit).

\subsubsection{Ray-casting}

This method of display is illustrated in Figure 1.7. Here, a series of parallel rays are projected through a $3 \mathrm{D}$ object onto a $2 \mathrm{D}$ image plane. The value of each pixel in this plane is then determined as a given function (the 
display model) of the values of the object voxels along the path of the associated ray (Fenster and Downey 1996; Robb 1995). Display models such as maximumintensity, minimum-intensity, maximum-gradient, or density-weighted projections are commonly used. This process results in an image which displays the anatomy in a translucent manner. A strong 3D impression of the anatomy can then be formed by generating a series of similar images, incrementally rotated through $360^{\circ}$, and viewing them as a ciné loop.

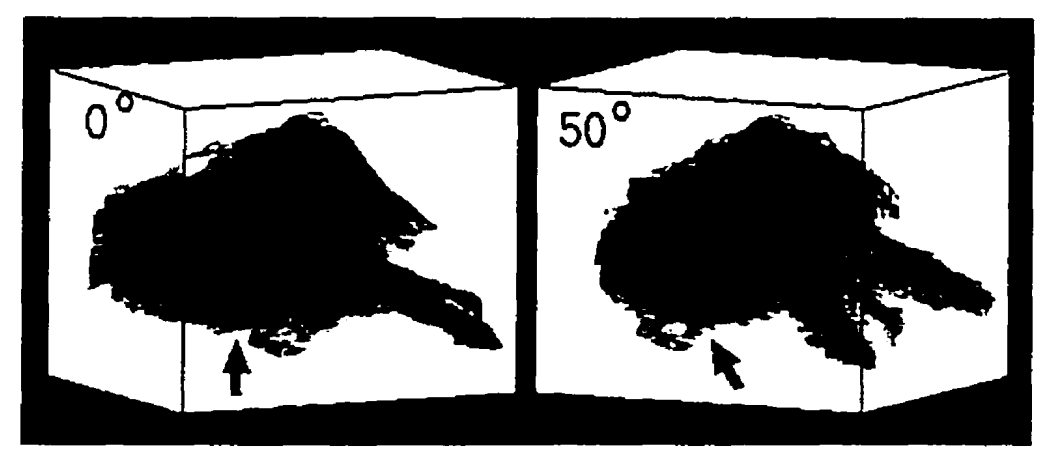

Figure 1.7 Minimum-intensity projection of a prostate and its attached seminal vesicles, shown for two different azimuthal angles. An arrow indicates the location of a tumour in each image.

\subsection{Thesis Outline}

\subsubsection{Overview}

The body of the thesis comprises the next four chapters, which describe how the specific goals listed in Section 1.5 were achieved. In Chapter 2, the development of the 3D ultrasound imaging system is described, and its imaging performance is evaluated using laboratory test phantoms. In Chapter 3 , the accuracy and precision of prostate volume measurements made in vitro is studied, using specimens obtained from cadavers. Next, in Chapter 4, the geometric distortions which may appear in reconstructed 3D images are theoretically analyzed, numerically simulated, and experimentally measured. 
Finally, in Chapter 5, an in vivo study is conducted to compare the intra- and inter-observer variability of prostate volume measurements performed via a $3 D$ ultrasound method to those performed via the conventional HWL method.

\subsubsection{Development of the 3D ultrasound imaging system}

Chapter 2 describes the development of a 3D ultrasound imaging system for imaging the prostate. The system is comprised of three elements: (i) a conventional ultrasound machine and TRUS probe; (ii) a custom-built, motorized assembly for rotating the probe under microcomputer control; and (iii) a microcomputer with an 8-bit video frame grabber for $2 \mathrm{D}$ image acquisition and subsequent 3D image reconstruction. The accuracy and precision of distance and volume estimation using this system, and the changes in image resolution caused by the $3 \mathrm{D}$ image reconstruction algorithm, are evaluated in vitro using a wire phantom and water-filled balloons.

This chapter is based on the paper entitled "A three-dimensional ultrasound prostate imaging system", by Shidong Tong, Dónal B. Downey, H. Neale Cardinal, and Aaron Fenster, which was published in Ultrasound in Medicine and Biology, vol. 22: 735-746; 1996.

As principal author, I designed and implemented the imaging system, wrote much of the image acquisition, reconstruction and processing software, measured and analysed the experimental data, and drafted most of the text. Dr. Downey (radiologist) provided clinical advice during the design and implementation phases, obtained clinical images, and wrote the associated text. Dr. Cardinal (colleague) assisted with the data analysis and in editing the text. Dr. Fenster (supervisor) provided overall guidance. In addition, Drs. Paul Picot and Ross Mitchell provided access to their image acquisition and processing software libraries, while Mr. John Miller built the motorized probe holder and wire phantom used for this work.

This system has been awarded United States Patent, Three-Dimensional Ultrasound Imaging System (Fenster et al. 1996), in which I am listed as a coinventor. The system was licenced to Life Imaging Systems, Inc., and forms the 
basis of their Sirus ${ }^{\mathrm{TM}}$ 3D ultrasound imaging system.

\subsubsection{Accuracy of prostate volume measurement in vitro}

Chapter 3 evaluates the accuracy and precision of the 3D ultrasound imaging system for measuring prostate volumes in vitro. Six prostates were obtained from cadavers and imaged. Their volumes were then measured from the 3D images via manual planimetry. In this method, the $3 D$ volume image of the prostate is "sliced" in the computer into a series of uniformly-spaced, parallel 2D images, and the cross-sectional area of the prostate in each slice is manually outlined interactively on a computer monitor with a "mouse". Henceforth, this method will be called the 3D US method. By comparing these results to the volumes measured by water displacement in a graduated cylinder, the accuracy and precision of the 3D US method were determined.

This chapter is based on the paper entitled "Accuracy of prostate volume measurements in vitro using three-dimensional ultrasound", by Tracy L. Elliot, Dónal B. Downey, Shidong Tong, Carolyn A. McLean, and Aaron Fenster, which was published in Academic Radiology, vol. 3: 401-406; 1996.

As co-author, I participated in scanning the prostates using the 3D ultrasound imaging system, performed the 3D image reconstructions and data analysis, and wrote the associated text. Dr. Elliot (radiology resident, medical student at the time) and Dr. Downey (radiologist) participated in scanning the prostates, performed the manual planimetry and the volume measurements by water displacement, and drafted most of the text. Dr. McLean (pathologist) provided pathology slides of the prostates, and Dr. Fenster (supervisor) provided overall guidance.

\subsubsection{Analysis of image distortion}

In Chapter 4, we determine the potential sources of geometric distortion in the reconstructed 3D ultrasound image for a rotational scan with a side-firing probe, and theoretically analyze the form and magnitude of these distortions quantitatively. Distortion measurements from numerically simulated and experimentally collected 
images are then used to verify the accuracy of the simple theoretical formulas for image distortion in the shape and length of a straight line, and in the size of an arbitrary cross-sectional area or object volume, as functions of the values of the scan parameters used by the 3D image reconstruction algorithm. We show that when the correct parameter values are used, all image distortions are theoretically zero, and we describe simple calibration procedures for determining these values accurately, and thereby reducing all image distortions to negligible levels.

This chapter is based on the paper entitled "Analysis of linear, area, and volume distortion in 3D ultrasound imaging", by Shidong Tong, H. Neale Cardinal, Dónal B. Downey, and Aaron Fenster, which has been submitted for publication in Ultrasound in Medicine and Biology.

As principal author, I carried out most of the theoretical analysis and all of the numerical simulations, experimental imaging scans, and data analysis, and drafted most of the text. Dr. Cardinal (colleague) assisted in the theoretical analysis and in editing the text, while Dr. Downey (radiologist) and Dr. Fenster (supervisor) provided overall guidance.

\subsubsection{Intra- and inter-observer variability}

Chapter 5 describes the results of a clinical study to evaluate of the intraand inter-observer variability of prostate volume measurements made by two methods: the HWL method and the 3D US method. Eight observers participated in the study, and measured fifteen 3D ultrasound prostate images, obtained from clinical patients in vivo. The height $H$, width $W$, and length $L$ of the prostate image was measured in transverse and sagittal cross-sections of the 3D images, which were used as a substitute for conventional 2D TRUS scans, in order to avoid repeated patient examinations, and manual planimetry of the 3D images was performed by using a "mouse" to outline the prostate image on computer image "slices" spaced $4 \mathrm{~mm}$ apart. This measurement procedure was conducted by each observer on each prostate, in an individually randomized order, over a 15-week period, and then repeated, in a different order, over a further 15-week 
period. All volume calculations were performed by the author at the end of the study, in order to avoid any observer bias due to previous results. An analysis of variance (ANOVA) of the measured prostate volumes was then performed to determine the intra- and inter-observer variability and reliability of each method.

This chapter is based on a paper entitled "Intra- and inter-observer variability and reliability of prostate volume measurement via 2D and 3D ultrasound imaging", by Shidong Tong, H. Neale Cardinal, Dónal B. Downey, and Aaron Fenster, which will be submitted for publication in Ultrasound in Medicine and Biology.

As principal author, I designed and coordinated the study, participated as one of the observers, performed most of the data analysis, and drafted most of the text. Dr. Cardinal (colleague) assisted in the data analysis and in editing the text. Dr. Downey (radiologist) provided the prostate images, participated as an observer, and gave clinical advice, while Dr. Fenster (supervisor) provided overall guidance. The ANOVA software was adapted from source code provided by Dr. Ross Mitchell.

\subsubsection{Summary and future work}

In Chapter 6, we summarize the principal results of Chapters 2 through 5, and outline some future work in the area of $3 \mathrm{D}$ ultrasound imaging. 


\subsection{References}

1. Baba K, Satoh K, Sakamoto S, Okai T, Ishii S. Development of an ultrasonic system for three-dimensional reconstruction of the fetus. J Perinat Med 17: 19-24; 1989.

2. Baum G, Greenwood I. Orbital lesion localization by three dimensional ultrasonography. NY State J Med 61: 4149-4157; 1961.

3. Belohlavek M, Foley DA, Gerber TC, Kinter TM, Greenleaf JF, Seward JB. Three- and four-dimensional cardiovascular ultrasound imaging: A new era for echocardiography. Mayo Clin Proc 68: 221-240; 1993.

4. Benson MC, Whang IS, Pantuck A, Ring K, Kaplan SA, Olsson CA, Cooner $W H$. Prostate specific antigen density: $A$ means of distinguishing benign prostatic hypertrophy and prostate cancer. J Urol 147:815-816; 1992a.

5. Benson MC, Whang IS, Olsson CA, McMahon DJ, Cooner WH. The use of prostate specific antigen density to enhance the predictive value of intermediate leveis of serum prostate specific antigen. J Urol 147: 817-821; $1992 b$.

6. Benson MC. Editorial: Prostate Specific Antigen. J Urol 152: 2046-2048; 1994.

7. Brawer MK, Aramburu EAG, Chen GL, Presten SD, Ellis WJ. The inability of prostate specific antigen index to enhance the predictive value of prostate specific antigen in the diagnosis of prostatic carcinoma. J Urol 150: 369-373; 1993.

8. Brinkley JF, Muramatsu SK, McCallum WD, Popp RL. In vitro evaluation of an ultrasonic three-dimensional imaging and volume system. Ultrasonic Imaging 4: 126-139; 1982.

9. Cho $\mathrm{ZH}$, Jones JP, Singh M. Foundations of Medical Imaging. New York: John Wiley \& Sons, Inc. ; 1993: 148-164.

10. Dekker $D L$, Piziali RL, Dong $E$ Jr. A system for ultrasonically imaging the 
human heart in three dimensions. Comput Biomed Res 7: 544-553; 1974.

11. Downey DB, Fenster A. Vascular Imaging with a Three-Dimensional Power Doppler System. AJR 165: 665-668; 1995.

12. Drago JR, Nesbitt JA, Badalament RA, York JP, Wientjes MG, Ashoton JJ, Smith JJ, Neff JC. Relative value of prostate-specific antigen and prostate acid phosphatase in diagnosis and management of adenocarcinoma of the prostate: Ohio State University experience. Urology 34: 187-192; 1989.

13. Elliott TL, Downey DB, Tong S, McLean CA, Fenster A. Accuracy of prostate volume measurements in vitro using three-dimensional ultrasound. Academic Radiology 3: 401-406; 1996.

14. Fenster A, Tong S, Sherebrin S, Downey DB, Rankin RN. Three-dimensional ultrasound imaging. Medical Imaging 1995: Physics of Medical Imaging, Proc SPIE 2432: 176-184, 1995.

15. Fenster A, Miller J, Tong S, Downey DB. Three-Dimensional Ultrasound Imaging System. The Unites States Patent \#5562095; October 1996.

16. Fenster A, Downey DB. 3-D ultrasound imaging: A review. IEEE Eng Med Biol, 41-51; Nov/Dec 1996.

17. Foster FS. Medical imaging with ultrasound. Physics in Canada 51: 182-189, $217 ; 1995$.

18. Greenleaf JF. Three-dimensional imaging in ultrasound. J Med System 6: $579-589 ; 1982$.

19. Greenleaf JF, Belohlavek M, Gerber TC, Foley DA, Seward JB. Multidimensional visualization in echocardiography: An introduction. Mayo Clin Proc 68: 213-220; 1993.

20. Guinan P, Bhatti R Ray P. An evaluation of prostate specific antigen in prostate cancer. J Urol 137: 686-889; 1987.

21. Halliwell $M$, Key $H$, Jenkins $D$, Jackson PC, Wells PNT. New scans from old: Ditial reformatting of ultrasound images. Br J Radiology 62: 824-829; 1989. 
22. Harada K, Igari D, Tanahashi $Y$. Grey scale transrectal ultrasonography of the prostate. J Clin Ultrasound 7: 45-49; 1979.

23. Hodges TC, Detmer PR, Burns DH, Beach KW, Strandness DE Jr. Ultrasonic three-dimensional reconstruction: In vitro and in vivo volume and area measurement. Ultrasound Med Biol 20: 719-729; 1994.

24. Höhne $K H$, Bomans $M$, Pommert $A$, Reimer $M$, Tiede $U$, Weibecke $G$. Rendering Tomographic Data: Adequacy of Methods for Different Modalities and Organs. In: Höhne KH, Fuchs H, Pizer SM, eds. 3D Imaging in Medicine: Algorithms, Systems, Applications. NATO ASI Series Vol. 60. Berlin: Springer-Verlag; 1990: 197-215.

25. Johns HE, Cunningham JR. The Physics of Radiology, 4th ed. Springfield, IL: Charles C Thomas Publisher; 1983: 633-640.

26. King DL, King DL Jr, Shao MYC. Three-dimensional spatial registration and interactive display of position and orientation of realtime ultrasound images. $J$ Ultrasound Med 9: 525-532; 1990.

27. King DL, King DL Jr, Shao MYC. Evaluation of in vitro measurement accuraccy of a three-dimensional ultrasound scanner. J Ultrasound Med 10: 77-82; 1991.

28. King WW, Williemeyer RM, Boyce WH, McKinney WM. Current status of prostatic echography. JAMA 226: 444-447; 1973.

29. Kirby RS, Christmas TJ, Brawer M. Prostate Cancer. London: Mosby; 1996: 77-92 (Chapter 8: Imaging and stanging of prostate cancer).

30. Lee F, Littrup PJ, Torp-Pedersen ST, Mettlin C, McHugn TA, Gary JM, Kumasaka GH, McLeary RD. Prostate cancer: Comparison of transrectal US and digital rectal examination for screening. Radiology 168: 389-394; 1988.

31. Lee F, Torp-Pedersen ST, Siders DB. The role of transrectal ultrasound in early detection of prostate cancer. CA Cancer J. Clin. 39: 337-360; 1989.

32. Littrup PJ. The development of a three dimensional prostate model. In: Lee F, 
McLeary RD, eds. The use of transrectal ultrasound in the diagnosis and management of prostate cancer. New York: Alan R Liss Inc.; 1987: 213-218.

33. Moritz WE, Pearlman AS, McCabe DH, Medema DH, Ainsworth ME, Boles MS. An ultrasounic technique for imaging the ventricle in three dimensions and calculating its volume. IEEE Trans Biomed Eng BME-30: 482-491; 1983.

34. National Cancer Institute of Canada: Canadian Cancer Statistics 1996. Toronto, Canada, January 1996. ISSN 0835-2976.

35. Picot PA, Rickey DW, Mitchell R, Rankin RN, Fenster A. Three-dimensional colour Doppler imaging. Ultrasound Med Biol 19: 95-104; 1993.

36. Rankin RN, Fenster A, Downey DB, Munk, PI, Levin MF, Vellet AD. Threedimensional sonographic reconstruction: techniques and diagnostic applications. ARJ 161: 695-702; 1993.

37. Rifkin MD. Ultrasound of the prostate. New York: Raven Press; 1988.

38. Rifkin MD. MRI of the prostate. Critical Reviews in Diagnostic Imaging 31: 223-262; 1990.

39. Rifkin MD, Zerhouni EA, Gatsonis CA, Quint LE, Paushter DM, Epstein Jl, Hamper U, Walsh PC, McNeil BJ. Comparison of Magnetic Resonance Imaging and Ultrasonography in Staging Early Prostate Cancer, Results of a Multi-Institutional Cooperative Trial. New England J of Med 323: 621-625; 1990.

40. Sawada $H$, Fujii J, Kato $K$, Onoe $M$, Kuno $Y$. Three dimensional reconstruction of the left ventricle from multiple cross sectional echocardiograms, Values for measuring left ventricular volume. $\mathrm{Br}$ Heart J 50: 438-442; 1983.

41. Sehgal CM, Broderick GA, Whittington R, Gorniak RJT, Arger PH. Threedimensional US and Volumetric Assessment of the Prostate. Radiology 192: 274-278; 1994.

42. Smith SW, von Ramm OT. High speed $3 D$ imaging with a two-dimensional array. Ultrasonic Imaging 11: 149; 1989. 
43. Smith EM, Resnick MI. Imaging of the prostate. In: Lepor H, Lawson RK, eds. Prostate diseases. Philadelphia: W. B. Saunders Company, 1993: 72-86.

44. Terris MK, McNeal JE, Stamey TA. Estimation of prostate cancer volume by transrectal ultrasound imaging. J Urol 147: 855-857, 1992.

45. Tong S, Downey DB, Cardinal HN, Fenster A. A three-dimensional ultrasound prostate imaging system. Ultrasound Med Biol 22: 735-746; 1996.

46. Watanabe $H$, Kaiho $H$, Tanaka $M$, Terasawa $Y$. Diagnostic applications of ultrasonotomography to the prostate. Invest Urol 8:548-559; 1971.

47. Watanabe $H$. History and applications of transrectal sonography of the prostate. Urol Clin North Am 16: 617-622; 1989.

48. Wingo PA, Tong T, Bolden S. Cancer statistics 1995. CA Cancer J Clin 45: 8-30; 1995. 


\section{DEVELOPMENT OF THE 3D ULTRASOUND IMAGING SYSTEM}

\subsection{Introduction}

Ultrasound imaging has proven valuable in the staging, monitoring and biopsy of prostate carcinoma. At present, transrectal ultrasound imaging is the most commonly used modality for imaging the prostate.

One of the major limitations of conventional 2D ultrasound images is its inherently two-dimensional character, since human anatomy is three-dimensional. Other disadvantages of $2 \mathrm{D}$ ultrasound imaging include: time-consuming in patient examination; inaccurate for quantitative volume measurements; and inappropriate for quantitative prospective or follow-up studies. Thus, we proposed a 3D ultrasound system for imaging the prostate which has potential to overcome these limitations.

This chapter describes: (i) the development of a prototype 3D ultrasound prostate imaging system; (ii) evaluation of the accuracy of distance and volume measurements using this system, and the changes in image resolution that may have been caused by the $3 \mathrm{D}$ image reconstruction process; (iii) imaging the prostate.

\subsection{Methods}

\subsubsection{Hardware: System Description}

The prototype 3D ultrasound imaging system consists of four elements: (i) a conventional ultrasound machine with a transrectal ultrasound transducer; (ii) a motor-driven assembly to hold and rotate the transducer, which is designed and built in our laboratories; (iii) a PC/AT microcomputer with an 8-bit video frame-grabber (model DT2851, Data Translation, Marlborough, MA); and, (iv) a Sun workstation (Sun Microsystems, Mountain View, CA). The first three elements are used for the image data acquisition, while the Sun workstation is used for image reconstruction, display, manipulation, and analysis (although these could also be done on a 
microcomputer).

The TRUS probe is mounted in the probe holder assembly so that, when activated, it is rotated around its long axis by a computer-controlled stepper motor (Figure 2.1). The 3D image data is then acquired by collecting a series of $2 D \mathrm{~B}$ mode images as the probe is rotated at constant speed. The probe rotates at a rate $0.2^{\circ}$ per step, with a motor speed ranging from $10 \mathrm{~ms}$ to $1 \mathrm{~s}$ per step. At pre-defined angular positions of the probe, a region of interest (ROI) of up to $256 \times 256$ pixels within the video image is digitized and stored in the random access memory (RAM) of the computer. These angular positions may be separated by any number of motor steps. The number of $2 \mathrm{D}$ images that can be acquired is limited only by the available extended memory capacity of the computer. For a typical acquisition, the probe is rotated through about $80^{\circ}$ while 100 images are collected. The entire data acquisition can be completed in 20 seconds.

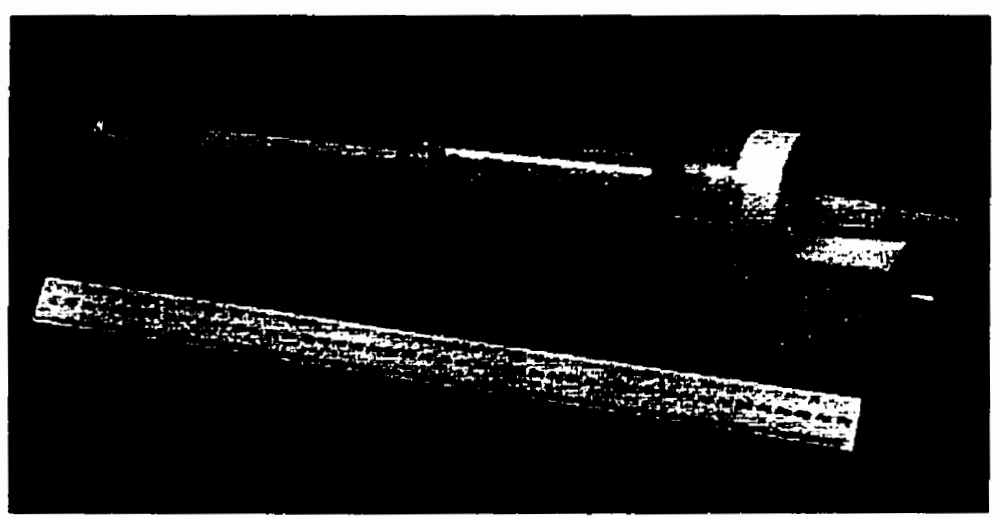

Figure 2.1 Photograph of a transrectal ultrasound (TRUS) probe, mounted on a computer-controlled motorized rotational mechanism. The stepping motor rotates the probe $0.2^{\circ}$ per step, at a speed of $10 \mathrm{~ms}$ to $1 \mathrm{~s}$ per step.

After a complete series of images is acquired, the image data are transferred via ethernet to a Sun SPARC 2 workstation for 3D image reconstruction and for display with $3 D$ visualization tools such as SunVision and the Sun TAAC-1 Application Accelerator (Sun Microsystems, Mountain View, CA).

For the results presented in this chapter, we used an ATL Ultramark 9 
ultrasound imaging system (Advanced Technology Laboratories, Bothell, WA) with a 5-MHz side-firing linear-array transducer. However, the system can be used with any ultrasound imaging system.

\subsubsection{Software: 3D Image Reconstruction}

Figure 2.2 shows a schematic diagram of the acquired images with respect to the transducer. The images are arranged as a fan of typically 10020 images, radiating outward from the ultrasound transducer, and evenly spaced over an arc of typically $80^{\circ}$. The reconstruction algorithm must produce a 3D image in which the relative orientation of the acquired images is preserved.

If the rotation axis of the transducer is designated as the z-axis, all the $2 \mathrm{D}$ image slices are perpendicular to the $x-y$ plane, so that the reconstruction algorithm for the $x-y$ plane and all the planes parallel to it is the same. The 3D reconstruction problem is therefore reduced to the $2 D$ problem of mapping the source data points, collected in cylindrical coordinates $(r, \theta, z)$, onto a regular grid of destination data points, in Cartesian coordinates $(x, y, z)$, i.e., for each value of $z$,

$$
P(r, \theta, z) \rightarrow P(x, y, z)
$$

Two distinct reconstruction methods were considered: source-oriented and destination-oriented. In the source-oriented method, for each gridpoint $(r, \theta)$ of the source image, the Cartesian coordinates $(x, y)=(r \sin \theta, r \cos \theta)$ are computed, and the associated image value is distributed to its nearest neighbours in the destination-image coordinate grid. However, near the axis, each destination grid point will receive contributions from several source points, due to oversampling; while, far from the axis, some destination grid points may not receive contributions from any source points, due to undersampling. Although radially renormalizing the image intensity can compensate for the effects of oversampling, it cannot fill in the holes left by undersampling. 


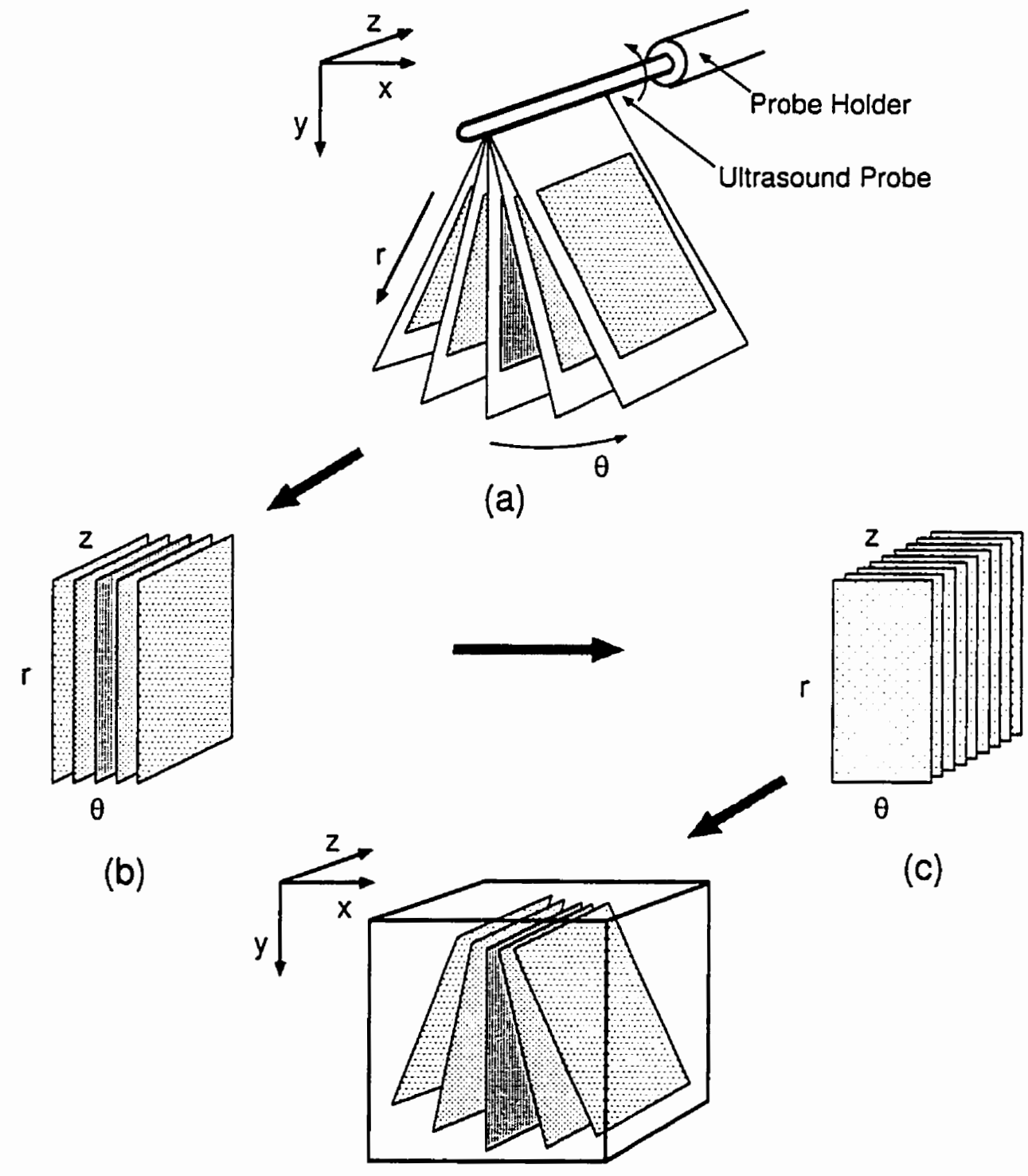

(d)

Figure 2.2 Schematic diagram outlining the process of $3 \mathrm{D}$ image acquisition and reconstruction. A series of $2 D$ images (a) is acquired while the probe is rotated in $\theta$ under computer control. Data from the resulting stack of $(r, z)_{0}$ images $(b)$ is rearranged into a stack of transaxial $(r, \theta)_{z}$ images $(c)$, which are then reconstructed into stack of $(x, y)_{2}$ images via a suitable interpolation scheme, thereby forming the reconstructed $3 D(x, y, z)$ image $(d)$, which can then be displayed, manipulated, and analyzed with standard image-processing software. 
In the destination-oriented method, for each gridpoint $(x, y)$ of the destination image, the polar coordinates $(r, \theta)=\left(\sqrt{ } x^{2}+y^{2}, \arctan (x / y)\right)$ are computed, and the associated image value is interpolated from its nearest neighbours in the source image. This method avoids the pitfalls of the previous method, and was therefore used for our system.

Three interpolation methods for the reconstruction were implemented: nearest-neighbour interpolation in both $r$ and $\theta$ ("nearest-neighbour interpolation"); nearest-neighbour interpolation in $r$ and linear interpolation in $\theta$ ("linear interpolation"); or, linear interpolation in both $r$ and $\theta$ ("bilinear interpolation"). Referring to Figure 2.3, the dashed lines represent the polar coordinates in which the points (pixel centroids) $a, b, c, d$, of the source image are located, and solid lines represent the Cartesian coordinates in which the point $p$ of the destination image grid is located. Let $P$ be the grey-scale value to be calculated, and $P_{a}, P_{b}, P_{c}, P_{d}$ be the grey-scale values at $a, b, c, d$, respectively. Then, if $f_{r}=\delta r / \Delta r$ and $f_{\theta}=\delta \theta / \Delta \theta$, where $\Delta \theta$ is the angular separation between successive 20 images, at $\theta_{i}$ and $\theta_{i+1}, \delta \theta$ is the angle between $\theta_{i}$ and $\rho, \Delta r$ is the radial separation between $r_{i}$ and $r_{i+1}$, and $d r$ is the distance between $r_{j}$ and $p$, in the case described by Figure 2.3, we have that, for nearest-neighbour interpolation:

$$
P=P_{a}
$$

while, for linear interpolation:

$$
P=\left(1-f_{\theta}\right) P_{a}+f_{\theta} P_{b}
$$

and, for bilinear interpolation:

$$
P=\left(1-f_{\theta}\right) f_{r} P_{a}+f_{\theta} f_{r} P_{b}+f_{\theta}\left(1-f_{r}\right) P_{c}+\left(1-f_{\theta}\right)\left(1-f_{r}\right) P_{d}
$$

Moreover, since the reconstruction algorithm for every $x-y$ plane is identical, the total reconstruction time can be reduced by creating a look-up table that designates which source pixels correspond to $a, b, c, d$ in Equations 2.2, and the associated values of $f_{\theta}$ and $f_{r}$, for every destination grid point $\rho$ in the ROI. The 
reconstructed 3D image is then built up by using this table repeatedly for each successive value of $z$.

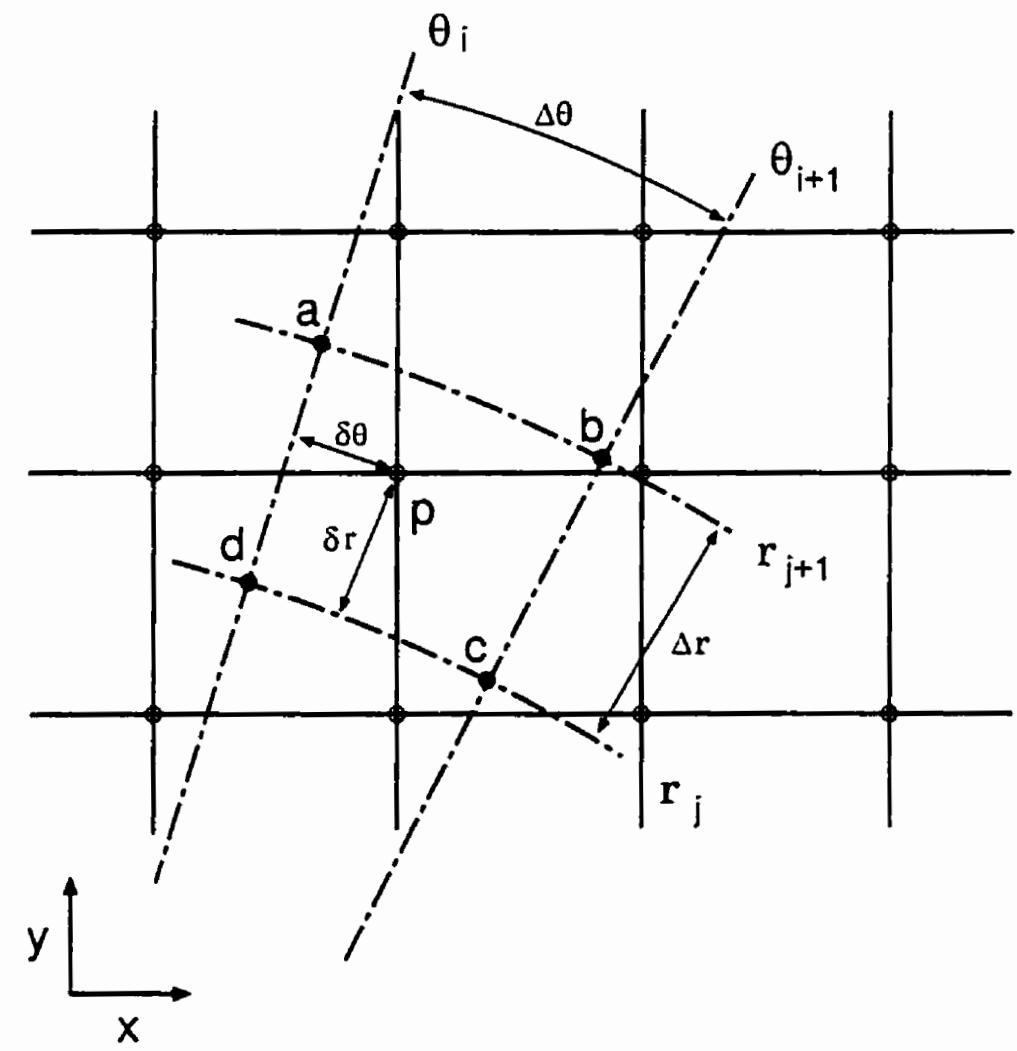

Figure 2.3 Schematic diagram illustrating the geometry used to describe the reconstruction algorithm. The source-image grid is in polar coordinates $(r, \theta)$ and the destination-image grid is in Cartesian coordinates $(x, y)$. The gray-scale value of (the pixel with centroid) $p$ in the destination image is calculated from those of its neighbors $a, b, c$, and $d$ in the source image.

\subsubsection{Evaluation of System Performance}

\subsubsection{Distance Measurement}

The accuracy of distance measurements was evaluated by imaging a 3D wire phantom. The phantom, built in our laboratory, is composed of four layers of 0.25 - 
$\mathrm{mm}$ diameter surgical wires, with 8 parallel wires per layer. The distance between layers is $10.00 \pm 0.05 \mathrm{~mm}$, as is the separation of wires within each layer.

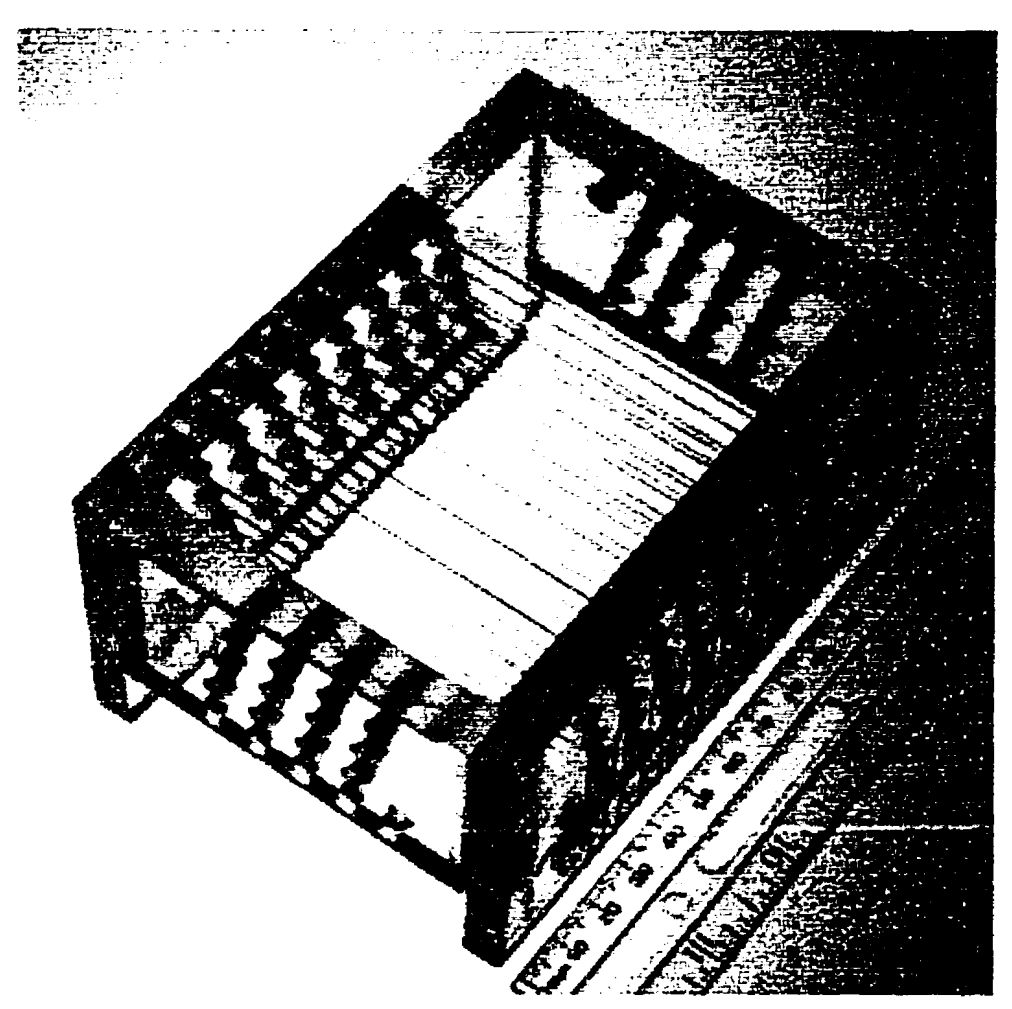

Figure 2.4 A photograph of the test phantom, which is composed of four layers of $0.25-\mathrm{mm}$ diameter wires, with 8 parallel wires per layer. The distance between layers is $10.00 \pm 0.05 \mathrm{~mm}$, as is the separation of wires within each layer.

For imaging, the wire phantom was immersed in a bath composed of a $7 \%$ glycerol solution in distilled water. This composition was chosen in order to make the speed of sound in the bath approximately $1540 \mathrm{~m} / \mathrm{s}$ (within $\pm 2 \%$ ), the soft-tissue calibration setting of the ATL Ultramark 9.

The wire phantom was scanned first with the wires placed parallel to the axis of rotation of the probe (the $z$ axis), which are designated as the $A$ images, and then with the wires oriented parallel to the $x$ axis, designated as the $B$ images. Nine $3 D$ scans were performed in each case. For each scan, $1002 \mathrm{D}$ images were collected over $60^{\circ}$. After reconstruction, the $A$ and $B$ images each contain 16 wire cross- 
sections, arranged in four layers. The distance of the first wire layer from the probe (i.e. the $y$-value of the layer) was about $27 \mathrm{~mm}$ for the first scan, and was increased by about $1 \mathrm{~mm}$ for each subsequent scan, so that, collectively, the nine scans contain wire images at $y$-values ranging from $27 \mathrm{~mm}$ to $66 \mathrm{~mm}$.

The separations between wire layers were then measured for each scan. The transverse and axial separations $\Delta x$ and $\Delta y$ were measured from the $A$ images, and the axial and lateral separations $\Delta y$ and $\Delta z$ from the $B$ images. To obtain three orthogonal separation measurements, the locations of the centroid of each wire image were determined automatically by a computer algorithm. The 3D volume was projected onto the $x-y$ plane to obtain the centroid coordinates $(x, y)$ of the 16 wire cross-sections of the $A$ images, and projected onto the $y-z$ plane to obtain the centroid coordinates $(y, z)$ of the 16 wire cross-sections of the $B$ images. The mean centroid separations were calculated by averaging the separations of adjacent centroids over the nine scans, obtaining the mean values of $\Delta x$ and $\Delta y$ from the $A$ images and the mean values of $\Delta y$ and $\Delta z$ from the $B$ images; the double determination of $\Delta y$ serving to double-check the results.

\subsubsection{Volume Measurement}

In order to evaluate the accuracy of volume measurements, we imaged a balloon filled with five different amounts of bath solution, and compared the measured volumes, derived from the $3 D$ ultrasound images, to the true volumes, derived by subtracting the mass of the empty balloon from that of the filled balloon and dividing the difference by the measured density $\left(1.0025 \mathrm{~g} / \mathrm{cm}^{3}\right)$ of the bath solution.

The balloon was filled with five different volumes of water bath solution ranging from about $23 \mathrm{~cm}^{3}$ to $66 \mathrm{~cm}^{3}$, and imaged with three different fields of view (and hence voxel sizes) at each volume, in the same manner and with the same bath composition as used for imaging the wire phantom. Each image data set consisted of $1002 \mathrm{D}$ images, scanned through $60^{\circ}$. 
After reconstruction, each 3D image was "sliced" to produce successive 2D images in planes spaced one voxel-thickness apart (about $0.2 \mathrm{~mm}$ ). For each $2 \mathrm{D}$ image, the balloon boundary was then manually outlined, and the number of pixels within the boundary determined. Multiplying the sum of these numbers (i.e. the total number of voxels within the balloon) by the voxel volume then yielded the measured volume of the balloon.

\subsubsection{Resolution Measurement}

The wire phantom was also used to evaluate the system resolution, or fullwidth at half-maximum (FWHM) of the cross-sectional image of a $B$ wire (one parallel to the $x$ axis), as a function of $y$, using the same data set as for the distance measurement (see above). In order to improve the precision of the FWHM value, the 4 cross-sections at the same $y$-value in each "slice" were superimposed for the central $16 y$-z "slices" of the 3D image. This was done for each of the $36 y$-values contained in the data set ( 9 scans $\times 4 y$-values per scan).

In order to determine whether the 3D reconstruction algorithm has degraded the image resolution, the same analysis was performed on the central $162 D$ images of each scan (distributed about $\theta=0$ ), and the results compared. This is feasible since although $\theta$ is non-zero for all but the central image, so that the image crosssections are distorted in the axial or $y$-direction, $\theta$ is between $\pm 4.8^{\circ}$, so that this distortion is negligible (less than $0.36 \%$ maximum, and $0.12 \%$ on average).

\subsubsection{D Imaging of the Prostate}

In a trial study, the prototype 3D TRUS system was used to image several patients scheduled for radical prostatectomy. For each patient, the probe was covered with a water-filled condom and inserted into the rectum, in the same manner as for a conventional TRUS examination. In each case, $1002 \mathrm{D}$ images were acquired while the probe was rotated through $80^{\circ}-100^{\circ}$, and subsequently reconstructed into a 3D image of a volume containing the prostate, the seminal vesicles, and their surrounding tissues. 
In order to assess the variation of $3 D$ image quality with the choice of interpolation method (nearest-neighbour, linear, or bilinear), a randomly selected data set was reconstructed with each method (via Equations. 2.2a, 2.2b, or 2.2c, respectively), and the same transaxial "slice" through the tumour was extracted for comparison. For each method, the reconstruction time was also recorded. The 3D image was viewed with SunVision and the TAAC-1 Application Accelerator, using two techniques.

The first technique, called "multi-slicing texture mapping" (Fishman et al. 1991; Robb 1995), displays the 3D image as a multi-sided object with the appropriate anatomy texture mapped on each face. The prostate can be viewed by "slicing" into the 30 image, interactively, to form a cross-sectional image of the prostate in any orientation.

The second technique, "ray-casting" (Fishman et al. 1991; Robb 1995), allows the prostate to be viewed as a translucent object. The prostate gland, including the seminal vesicles, is manually outlined in the original 3D image, and a new 3D image containing only the prostate gland and seminal vesicles is then created by the software. Here, a minimum-intensity ray-casting projection technique was applied to create translucent images of the prostate at different viewing angles, which were then stored for later viewing as a cine loop.

\subsection{Results}

\subsubsection{Distance measurement}

The results of the distance measurement study are shown in Table 2.1. This table shows that distance measurements made with our 3D ultrasound system have an accuracy of about $1.0 \%$, since the mean measured wire separation is $10.10 \mathrm{~mm}$ and true mean wire separation is $10.00 \mathrm{~mm}$. The precision of an individual separation measurement is accurate to about $1 \%$ for the $A$ images and $2-3 \%$ for the $B$ images. 
Table 2.1 Distance study results. A wire phantom was imaged with $(A)$ the wires oriented laterally (i.e. parallel to the $z$ or probe axis), and $(B)$ the wires oriented transversely (i.e. parallel to the $x$ axis). For each data set, the separation of adjacent wires was measured in the two orthogonal directions for the 16 central "slices" of the 3D image, yielding the measured means and standard deviations listed. By construction, the actual mean and standard deviation of $\Delta x, \Delta y$, and $\Delta z$ is $10.00 \pm 0.05 \mathrm{~mm}$.

\begin{tabular}{ccc}
\hline $\begin{array}{c}\text { Image } \\
\text { Data Set }\end{array}$ & $\begin{array}{c}\text { Distance } \\
\text { Measured }\end{array}$ & $\begin{array}{c}\text { Measured Distance } \\
\text { Mean } \pm \text { S.D. (mm) }\end{array}$ \\
\hline$A$ & $\Delta x$ & $10.11 \pm 0.11$ \\
& $\Delta y$ & $10.10 \pm 0.06$ \\
\hline$B$ & $\Delta y$ & $10.12 \pm 0.17$ \\
& $\Delta z$ & $10.07 \pm 0.29$ \\
\hline
\end{tabular}

Table 2.2 Volume study results. A balloon, filled with five different amounts of bath solution ( $7 \%$ glycerol in distilled water) was imaged using three different fields-of-view (and hence voxel sizes). For each amount, the mean and standard deviation of the three corresponding volume measurements is compared to the true volume, derived from the net balloon mass and the density $\left(1.0025 \mathrm{~g} / \mathrm{cm}^{3}\right)$ of the bath solution. The rms error is $0.9 \pm 1.7 \%$.

\begin{tabular}{ccc}
\hline \multirow{2}{*}{$\begin{array}{c}\text { True Volume } \\
\left(\mathrm{cm}^{3}\right)\end{array}$} & \multicolumn{2}{c}{ Measured Volume } \\
\cline { 2 - 3 } 23.14 & $22.81 \pm 0.33$ & $-1.4 \pm 1.4$ \\
35.79 & $35.49 \pm 0.72$ & $-0.8 \pm 2.0$ \\
41.69 & $41.27 \pm 0.65$ & $-1.0 \pm 1.6$ \\
49.66 & $49.84 \pm 0.62$ & $0.4 \pm 1.2$ \\
65.84 & $66.31 \pm 1.29$ & $0.7 \pm 2.0$ \\
\hline \hline
\end{tabular}




\subsubsection{Volume measurement}

The results of the volume measurement study are shown in Table 2.2. Since each volume was imaged at three different fields of view (and hence different voxel sizes), the measured volumes shown represent the average and standard deviation of the three volume estimates made for each true volume listed. From this table, we see that the volume measurements have a root-mean-square (rms) accuracy of $0.9 \%$ and an rms precision of $1.7 \%$. Also, a least-squares regression through the origin resulted in a best-fit line with a slope of $1.0004 \pm 0.0039$ and a correlation coefficient of 0.999970 .

\subsubsection{Resolution measurement}

Figure 2.5 shows the FWHM (in two directions) of the wire images vs the axial distance $y$ from the probe to the wire plane. The results in Figure 2.5a were determined from the 16 central "slices" of the reconstructed 3D image, and those in Figure 2.5b from the 16 central 2D images before reconstruction. In both cases, the FWHM is not symmetric, being much smaller, and independent of $y$, in the axial (or $y$ ) direction than it is in the lateral (or $z$ ) direction, where it exhibits a dual focalzone pattern of variation with $y$. In the $3 D$ case (Figure 2.5a), the FWHM has a mean axial value of $0.90 \pm 0.02 \mathrm{~mm}$, and a mean lateral value of $3.57 \pm 0.07 \mathrm{~mm}$, while in the $2 \mathrm{D}$ case (Figure 2.5b), the FWHM has a mean axial value of $0.83 \pm$ $0.02 \mathrm{~mm}$, and a mean lateral value of $3.47 \pm 0.07 \mathrm{~mm}$ (over the range of $y$-values shown). Figure 2.6 shows that, in both directions, the excess of the 3D FWHM's over their 2D counterparts is essentially independent of $y$. Figure 2.6a shows that the axial excess is always positive, with a mean value of $0.069 \pm 0.023 \mathrm{~mm}$, while Figure 2.6b shows that the lateral excess is almost random in sign, with a mean value of $0.097 \pm 0.107 \mathrm{~mm}$. Thus, the axial resolution is only slightly $(8 \pm 3 \%)$ degraded by the 3D reconstruction algorithm, while the lateral resolution is negligibly $(3 \pm 3 \%)$ degraded. In practice, these changes in resolution would be nearly imperceptible. 

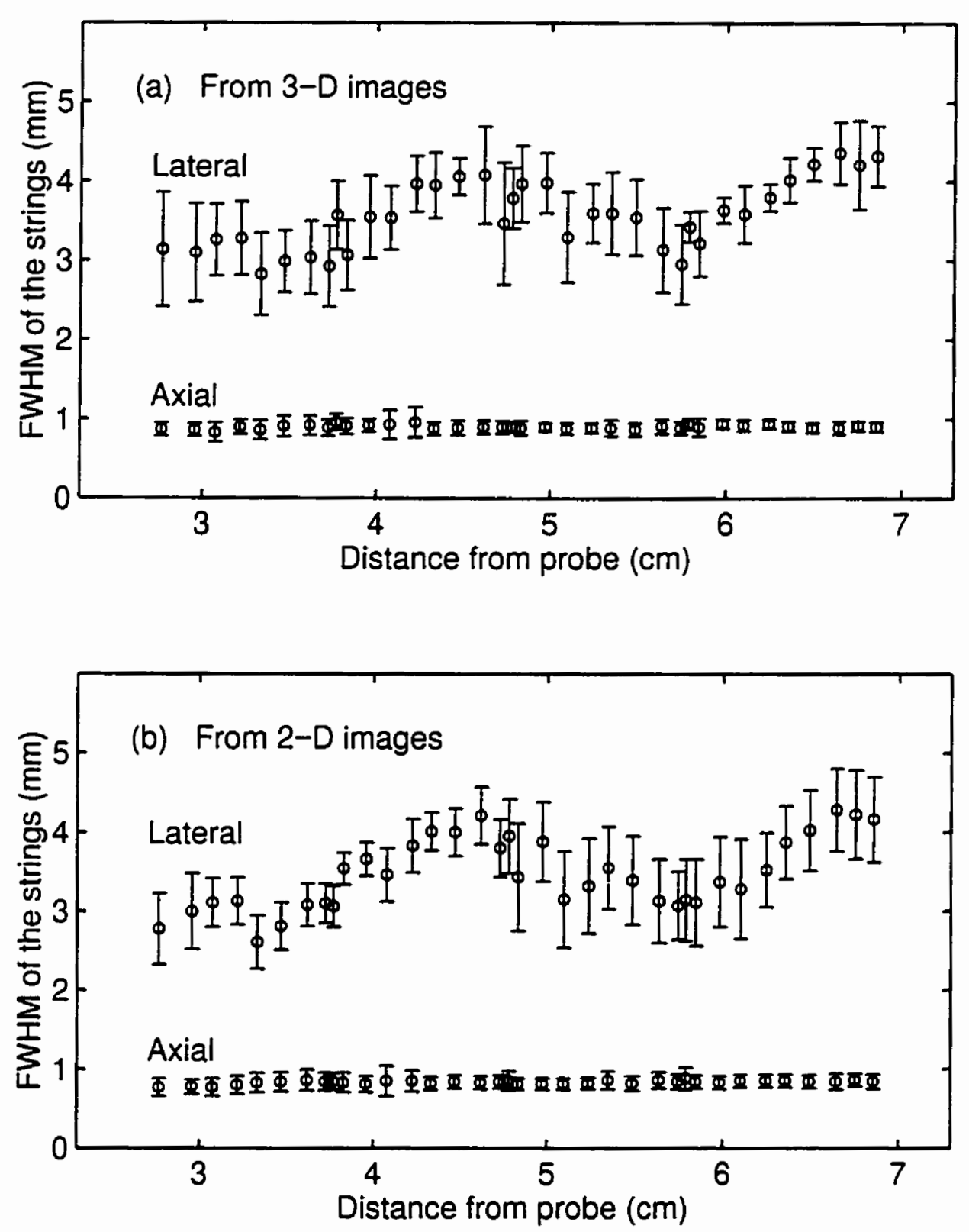

Figure 2.5 Full-width at half-maximum (FWHM) of the wire cross-sectional images in the lateral (or $z$ ) and axial (or $y$ ) directions for wire layers at various distances $y$ from the probe, averaged over (a) the central 16 "slices" of the reconstructed $3 D$ image, and (b) the central 16 original $2 D(r, z)$ images. 

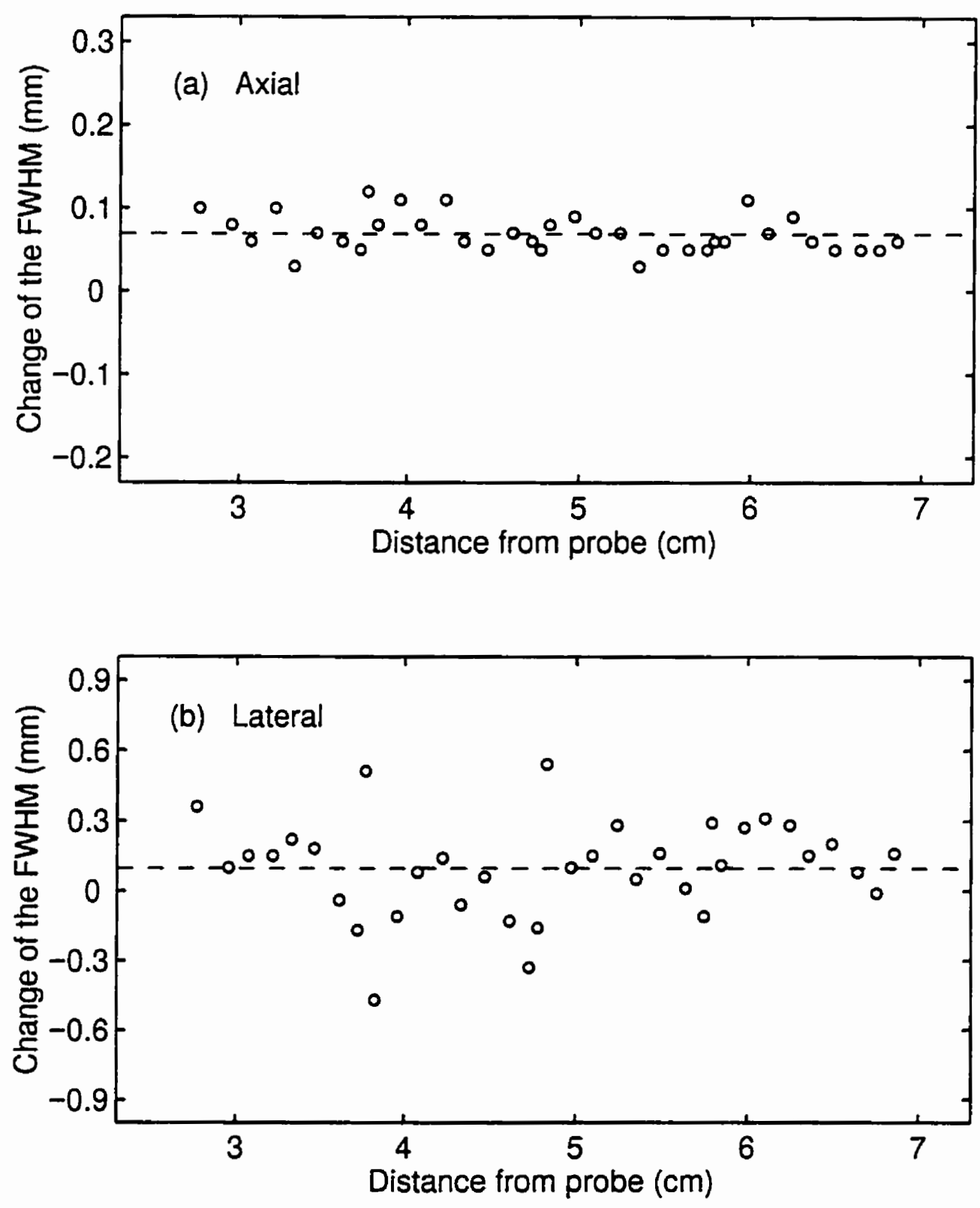

Figure 2.6 The increase in the full-width at half-maximum (FWHM) of the wire cross-sectional images caused by the 3D reconstruction algorithm (calculated as the difference between the $3 D$ and 2D FWHM's shown in Figure 2.5), for wire layers at various distances $y$ from the probe. The dashed lines show a mean increase of (a) $0.069 \pm 0.023 \mathrm{~mm}$ in the axial FWHM (an $8 \% \pm 3 \%$ increase), and (b) $0.097 \pm 0.107 \mathrm{~mm}$ in the lateral FWHM (a $3 \% \pm 3 \%$ increase). 


\subsubsection{D imaging of the prostate}

Figure 2.7 to Figure 2.9 show a 3D image of the prostate, "sliced" in different planes to reveal the tumour and other relevant structures. Although the original $2 D$ images were obtained in sagittal planes, this patient's B1 tumour $(T)$ is well demonstrated on the reconstructed transaxial plane in Figure 2.7. By "slicing" parasagittally into the prostate, Figure 2.8, we can now see the tumour in both transaxial and sagittal planes, and see that it has not invaded either the periprostatic fat (F) or seminal vesicles (S). In Figure 2.9, the prostate has been rotated superiorly and "sliced" in a paracoronal plane through both the tumour $(T)$ and left seminal vesicle (S), so that the tumour is now visible in 3 planes and seminal vesicle invasion is excluded. The coronal plane, which is not available with conventional TRUS, demonstrates the urethra (U), tumour (T), seminal vesicles (SV) and vasa deferentia (V). 


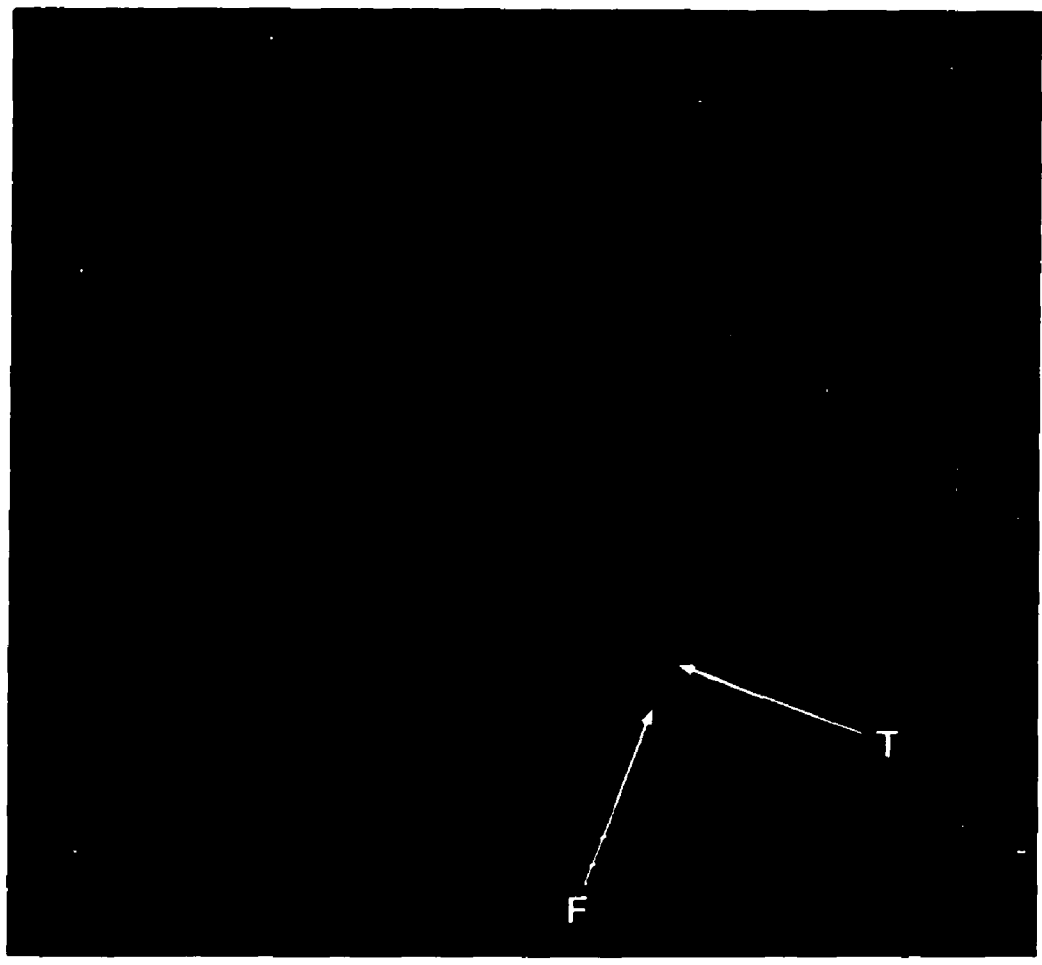

Figure 2.7 3D images of a prostate are "sliced" to reveal the tumor (T) and the peri-prostatic fat $(F)$. Although the original 20 images were obtained in sagittal planes, the patient's $B 1$ tumor $(T)$ is well demonstrated on the reconstructed transaxial plane. 


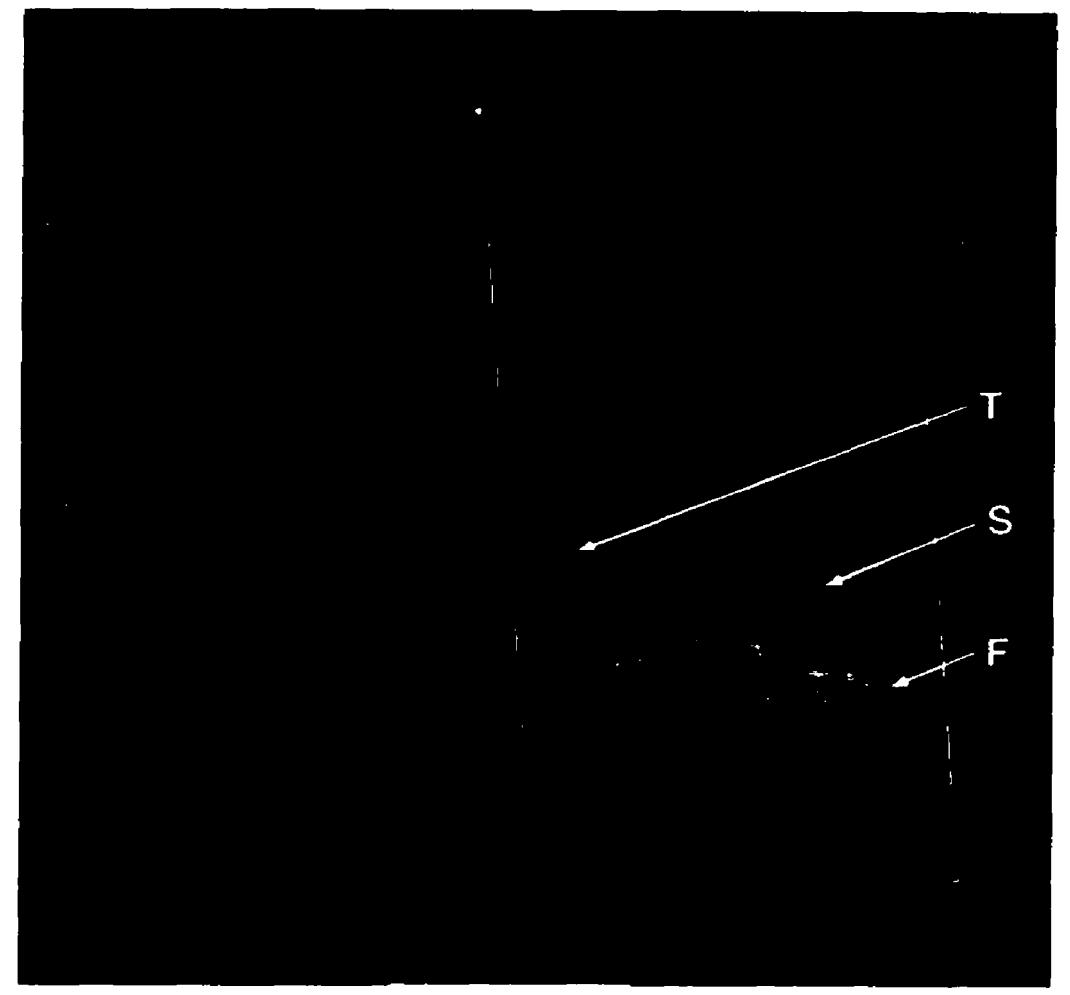

Figure 2.8 By "slicing" into the prostate, the tumor $(T)$ can be seen in both transaxial and sagittal planes. It is seen that the tumor has not invaded either the peri-prostatic fat $(F)$ or seminal vesicles $(S)$. 


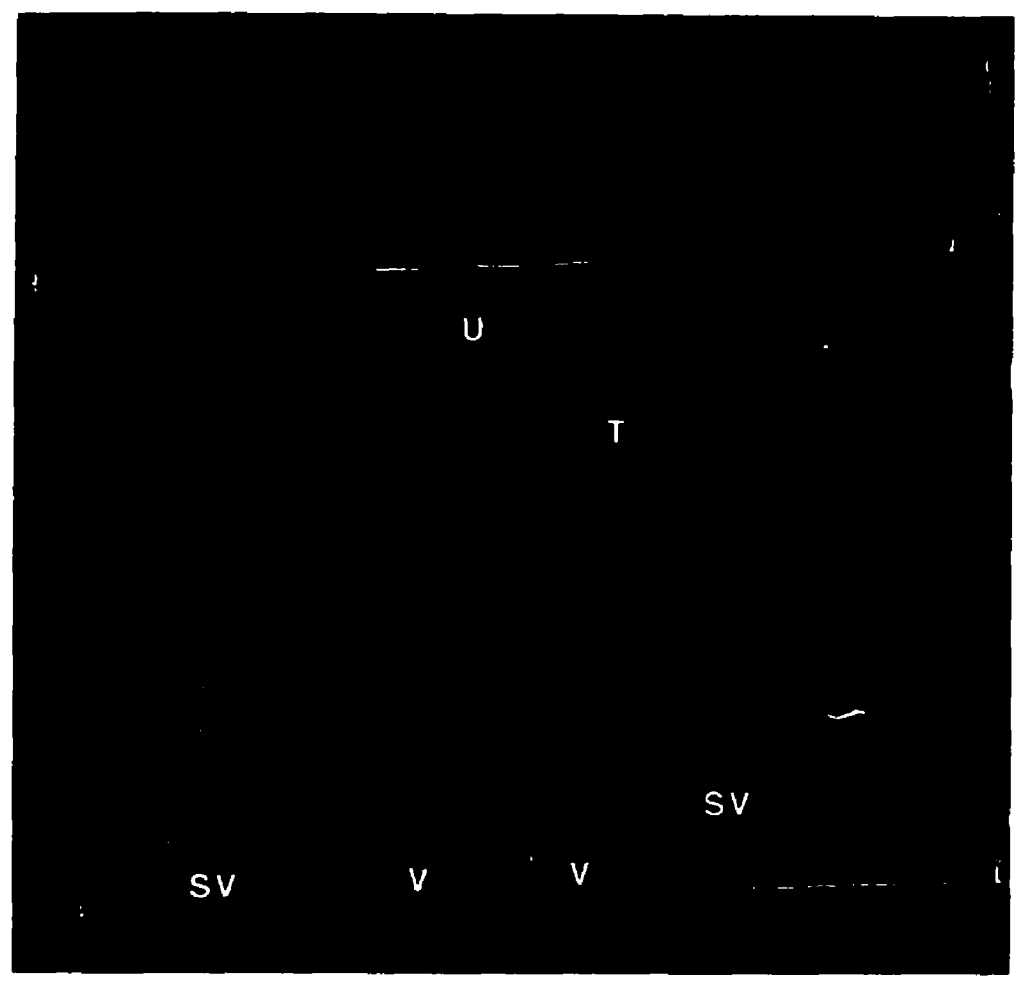

Figure 2.9 The coronal plane, which is not available with conventional transrectal ultrasound (TRUS), demonstrates the urethra (U), tumor (T), seminal vesicles (SV), and vasa deferentia (V). 
Figure 2.10 shows the corresponding hematoxylin- and eosin-stained slide of the tumour following radical prostatectomy. The slide confirms the location of the prostate tumour shown in the 3D images of Figure 2.7.

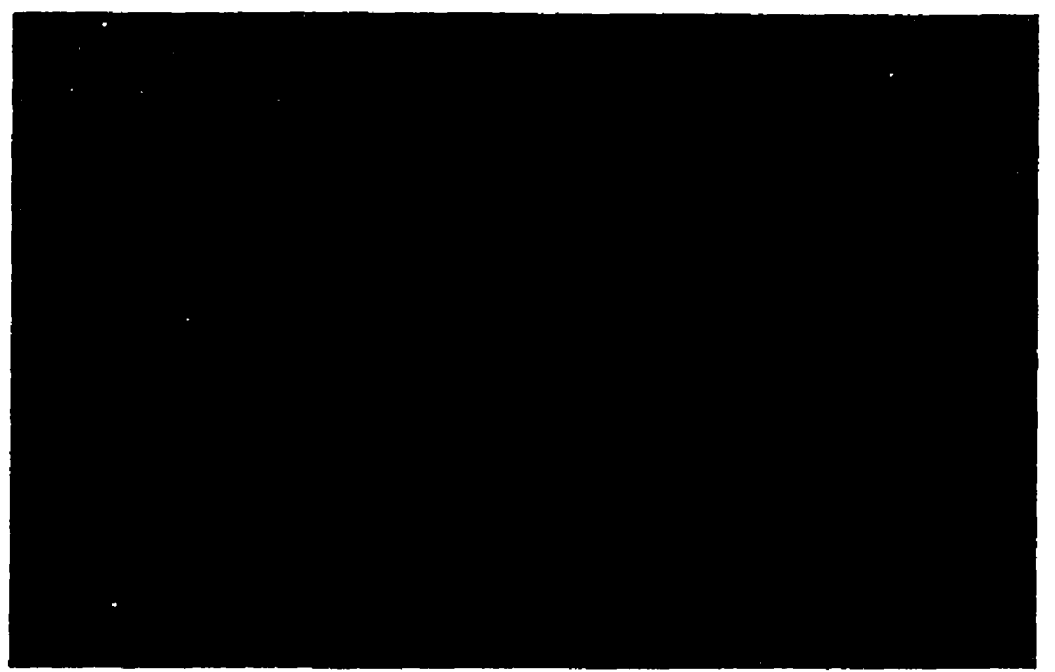

Figure 2.10 The hematoxylin- and eosin-stained slide of the tumor as shown in Figure 2.7, following radical prostatectomy. 
Figure 2.11 shows four views of the translucency rendered prostate from four different angles. An arrow points to the hypoechoic tumour in each view. When a sufficient number of views of the prostate from different angles have been created, the sequence of images can be played back continuously as a cine loop to provide a $3 D$ impression of the anatomy.

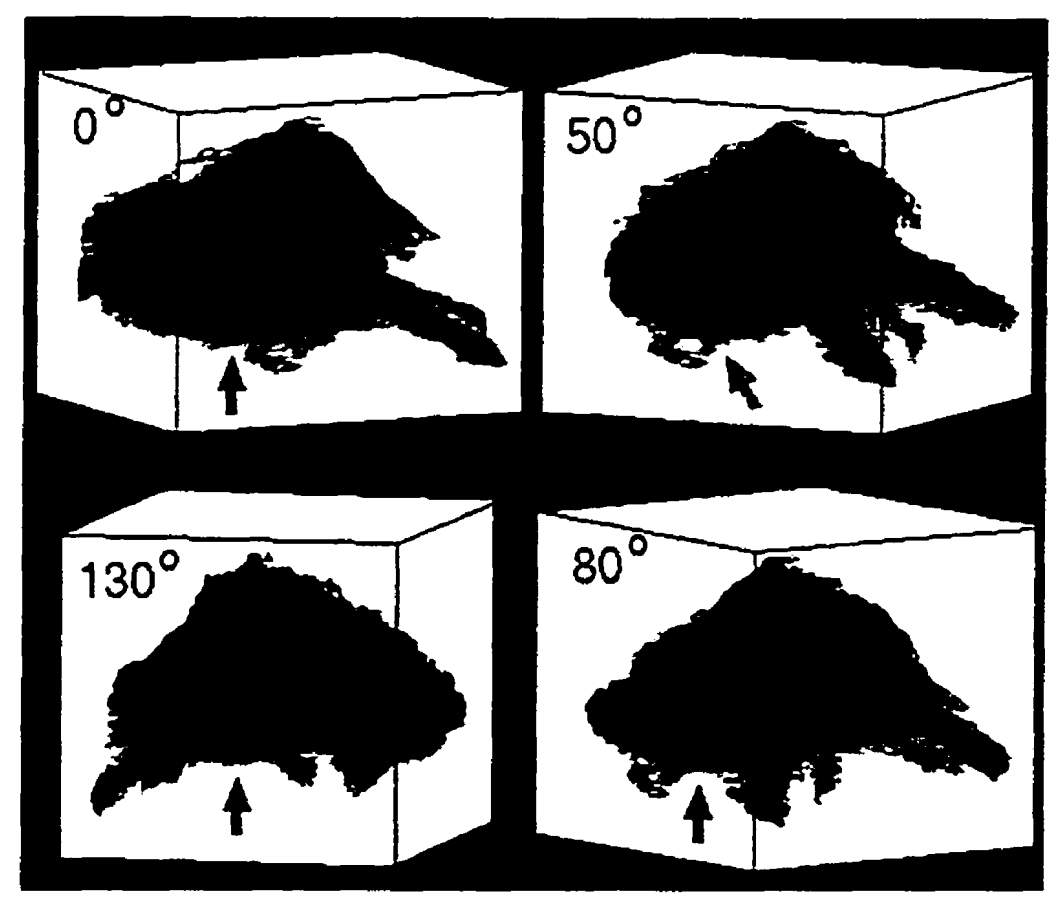

Figure 2.11 Four views of a translucency-rendered prostate image, at four different angles, derived from the same image data used for Figure 2.7. The arrow points to the hypoechoic tumor. 
Figure 2.12 to Figure 2.14 show cross-sectional "slices" from a 3D image reconstructed by each of the three interpolation methods previously described. The image reconstructed via nearest-neighbour interpolation (Figure 2.12) is severely pixelated, while the images reconstructed via linear and bilinear interpolation (Figure 2.13 and Figure 2.14) are only moderately pixelated, the former more so than the latter, in agreement with our intuitive expectations.

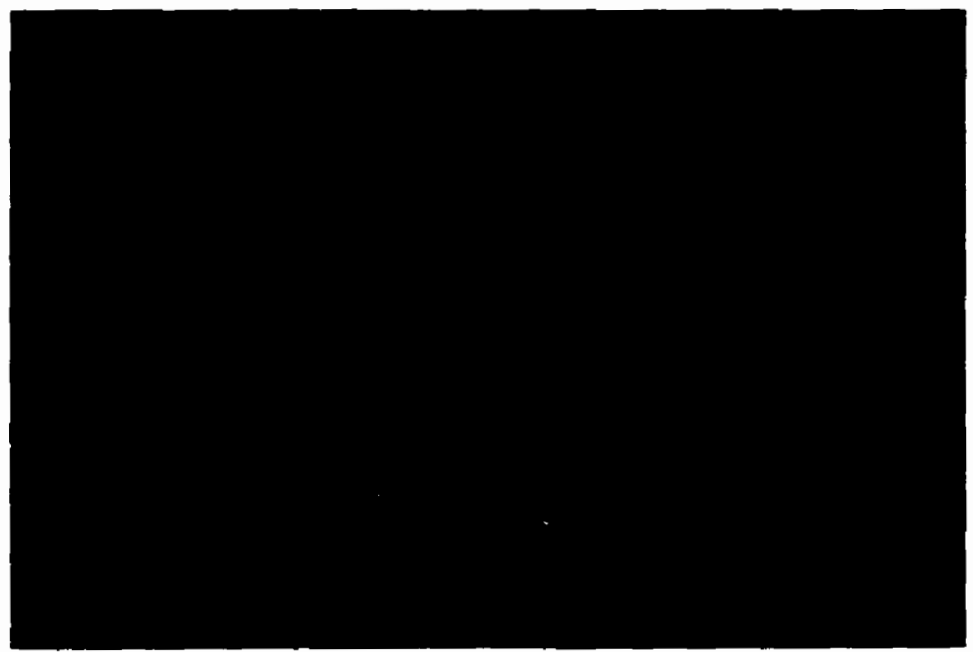

Figure 2.12 Cross-sectional slices from a $3 D$ image of the prostate reconstructed using nearest-neighbor interpolation. 


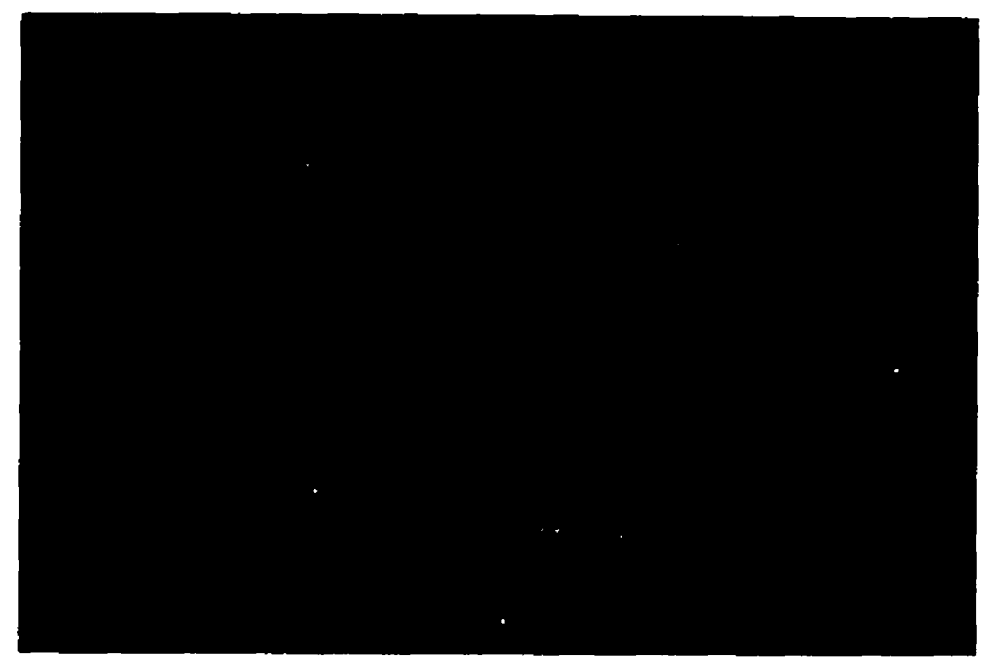

Figure 2.13 Cross-sectional slices from a 3D image of the prostate reconstructed using linear interpolation.

Figure 2.14 Cross-sectional slices from a 3D image of the prostate reconstructed using bilinear interpolation. 
However, although using more complex interpolation improves the image appearance, it also increases the reconstruction time. In Table 3 we tabulate the computation time needed for a Sun SPARC 2 workstation to reconstruct a 3D image of size $310 \times 190 \times 230$ pixels from $1002 \mathrm{D}$ images of size $230 \times 176$, covering an angle of $80^{\circ}$, for each of the interpolation methods used. From this table, we see that using linear interpolation instead of nearest-neighbour interpolation increases the reconstruction time by a factor of 1.63 , and that using bilinear instead of linear interpolation increases it by a further factor of 1.65 , to a total time of about 1.9 minutes.

Table 2.3 CPU times for reconstructing a 3D image of size $310 \times 190 \times 230$ pixels from 10020 images of size $230 \times 176$ pixels, covering an angle of $80^{\circ}$, using a Sun SPARC 2 workstation (Sun Microsystems, Mountain View, CA), using each of the interpolation methods described by Equations 2.2.

\begin{tabular}{cc}
\hline Interpolation Method & CPU Time (s) \\
\hline Nearest-Neighbor & 42.6 \\
Linear & 69.5 \\
Bilinear & 114.7 \\
\hline \hline
\end{tabular}

\subsection{Discussion}

We have developed a 3D ultrasound system for imaging the prostate, which is comprised of four elements. The first three, (i) a conventional ultrasound machine with a TRUS transducer, mounted in (ii) a custom-designed and fabricated motordriven assembly, controlled by (iii) a microcomputer with an 8-bit video framegrabber, are used for the image data acquisition. The fourth element, a workstation, is then used for the 3D image reconstruction, display, and manipulation. Except for the second element, these are all readily available commercially.

We remark that although the system described here was developed to use a 
PC (in the examination room) for data acquisition, and a workstation (elsewhere) for image reconstruction, display, manipulation, and analysis, it has recently been adapted to use a Macintosh computer to perform both functions.

The results of this preliminary study demonstrate that our 3D ultrasound imaging system greatly benefits both patients and clinicians, by drastically reducing the time required to complete an examination. Once acquired, the 3D images can be cheaply stored electronically for later review and analysis, with no loss of information of the interior structure of the prostate.

Image cross-sections may be displayed in a format similar to that of conventional 2D TRUS images, already familiar to radiologists, but with the capability of "slicing" the 3D volume in any plane. This enables the prostate to be viewed in orientations, such as in the coronal plane, which are unavailable with conventional 2D TRUS, due to the restrictions imposed by patient anatomy. In addition, 3D presentation of the data, via techniques such as ray-casting, is easily accomplished (Fishman et al. 1991; Robb 1995), relieving the radiologist of the arduous and time-consuming task of developing a 3D impression of the prostate anatomy by mentally integrating a series of $2 D$ images.

Also, the 3D image data may be analyzed to provide direct measurements of quantities such as distance or volume. In particular, the accurate determination of tumour volume is crucial for the accurate staging of prostate cancer, for the interpretation of PSA levels, or for monitoring the effects of chemotherapy on BPH, etc. Our current system permits both distances and volumes to be estimated with an accuracy of about $\pm 1 \%$ (comparable to the experimental uncertainty of the distance measurements).

At present, volume measurements are carried out by the manual segmentation of a series of $2 \mathrm{D}$ image slices via boundary outlining, i.e. manual planimetry. Although this technique is sufficiently accurate, it is tedious and timeconsuming to perform (particularly at the slice-spacing of $0.2 \mathrm{~mm}$ used here); furthermore, it neglects the image correlation between neighbouring slices. It would 
therefore be highly desirable to develop a (semi-)automatic three-dimensional segmentation algorithm, which could determine tumour or prostate volumes directly, e.g., by simply counting the number of voxels "inside" the tumour or prostate. For the greatest accuracy, the voxels should therefore be as small as possible (within the limits imposed by system resolution), implying that the slice spacing should be comparable to the pixel dimensions of the cross-sectional images, yielding roughly cubical voxels (and hence, as a fringe benefit, roughly isotropic linear measurement accuracy). Our system was designed with this development in mind.

The image data is originally acquired as a set of $2 D(r, z)$ images at uniformly spaced angles of probe rotation $q$. For each value of $z$, the set of $2 \mathrm{D}$ image data at polar coordinates $(r, \theta)$ is interpolated to generate a corresponding set of $2 D$ image data at Cartesian coordinates $(x, y)=(r \sin \theta, r \cos \theta)$ over a rectangular $R O I$, using either nearest-neighbour interpolation in both $r$ and $\theta$ ("nearest-neighbour interpolation", Figure 2.12, nearest-neighbour interpolation in $r$ and linear interpolation in $\theta$ ("linear interpolation", Figure 2.13, or linear interpolation in both $r$ and $\theta$ ("bilinear interpolation", Figure 2.14. Subjectively, there is a dramatic improvement in image quality in going from nearest-neighbour to linear interpolation (Figure 2.12 to Figure 2.14), and a modest improvement in going from linear to bilinear interpolation (Figure 2.13 to Figure 2.14). This is to be expected, since, far from the probe axis $(r=0)$, the azimuthal interval $r \Delta \theta$ in the source image can become much (here, up to three times) larger than the Cartesian grid interval $\Delta x=\Delta y$ in the destination image (which equals the radial interval $\Delta r$ in the source image), making angular interpolation much more important than radial interpolation there.

The 3D image is then assembled by stacking the interpolated $2 D(x, y)$ images in the $z$ direction. In order to assess any loss of resolution introduced by the interpolation process, the average image resolution (here, FWHM of the point spread function) in the $(y, z)$ plane (near $x=0$ ) was compared to that in the original $(r, z)$ images (near $\theta=0$ ) (since $y \approx r$ near $x=0, \theta=0$ ). Using linear interpolation, 
the loss of resolution is very slight $(8 \pm 3 \%$ ) in the axial (or $y$ ) direction and negligible $(3 \pm 3 \%)$ in the lateral (or $z$ ) direction. With bilinear interpolation, we would expect negligible losses in both directions.

We chose to acquire the $2 \mathrm{D}$ images by using a frame-grabber to digitize the video output directly from the (analog) video output port of the ultrasound machine, rather than digitizing a videotape recording of this output, in order to avoid any signal degradation by the videotape. An even better approach would be to utilize the output before its digital-to-analog conversion by the ultrasound machine (thus eliminating two data conversions), but this type of capability would require design modifications to the ultrasound machine.

Another improvement would be to write the image data directly to disk while the scan is in progress, instead of storing it in RAM until the scan is complete, and then writing it to disk. This would eliminate the restrictions on the size of the ROI and/or the number of $2 D$ images (i.e. rotational steps) imposed by the size of the available RAM.

Although our 3D ultrasound imaging system was developed specifically for imaging the prostate, it has been successfully modified to image other organs, such as the eye (Downey et al. 1996), the breast (Downey and Fenster 1995), and the kidney (Downey and Fenster 1995). 


\subsection{References}

1. Downey DB, Nicolle DA, Levin MF, Fenster A. Three-dimensional ultrasound imaging of the eye. Eye $10: 75-81 ; 1996$.

2. Downey $\mathrm{DB}$, Fenster $\mathrm{A}$. Vascular imaging with a three-dimensional power Doppler system. AJR 165: 665-668; 1995.

3. Fenster A, Miller J, Tong S, Downey DB. Three-dimensional ultrasound imaging system. The United States Patent \#5562095; October 8, 1996.

4. Fishman EK, Magid D, Ney DR, Chaney EL, Pizer SM, Rosenman JG, Levin DN, Vannier MW, Kuhlman JE, Robertson DD. Three-dimensional imaging, Radiology 181: 321-337; 1991.

5. Robb RA. Three-dimensional biomedical imaging: Principles and practice. New York: VCH Publishers Inc.; 1995. 


\section{ACCURACY OF VOLUME MEASUREMENTS IN VITRO}

\subsection{Introduction}

Recent advances in prostate imaging have improved our ability to diagnose and manage prostate disease, however, current prostate imaging methods are sub-optimal and have many clinical problems. The ideal prostate imaging technique would be user-compliant, inexpensive, fast, convenient for the patient, accurate and precise. It would, among other things, allow rapid imaging of the prostate; allow accurate measurements of the volume of the prostate and tumors; allow accurate staging of prostate cancer; have the ability to guide interventional procedures for diagnostic biopsy and therapeutic applications; and, have the ability to assess response to treatment of prostate cancer.

Today, transrectal ultrasound imaging (TRUS) has the potential to meet these criteria. Transrectal ultrasound imaging is currently commonly performed because it is more readily available, less expensive, and can accurately guide biopsies. However, it is far from ideal. Examinations are time-consuming, both for the patient and the physician. Qualitative information is sub-optimal. Although TRUS is the most commonly used technique for measuring prostate volume, it is both cumbersome and inaccurate (Littrup et al. 1991).

We have developed a 3D ultrasound system for imaging the prostate. The 3D image can be viewed in multiple planes in different orientations so that otherwise inaccessible imaging planes such as coronal, oblique, in addition to biplanar and triplanar perspectives, can be readily visualized (Tong et al. 1996, Rankin et al. 1993). The data acquisition times are shorter, making the procedure efficient and therefore, less uncomfortable for patients than conventional $2 D$ ultrasound imaging exams. Volume measurement of whole prostates and focal lesions within can be obtained using special software. Phantom studies show that these techniques allow more accurate volume estimation than standard 2D ultrasound (Tong et al. 1996, Sehgal et al. 1994). All of these factors combine to suggest $3 D$ ultrasound will have significant 
clinical utility. However, its actual utility is currently unknown and several facets of these systems must be assessed before extensive testing is done on humans.

In Chapter 2, we have evaluated the accuracy of volume estimation on laboratory phantoms using our 3D imaging system. The focus of this chapter is to evaluate in vitro measurement of the prostate volume. An evaluation of in vivo measurements of prostate volume will be presented in Chapter 5 .

\subsection{Materials and Methods}

\subsubsection{Cadaver specimens}

Six prostates, with seminal vesicles and some periprostatic fat attached, were harvested from fresh cadavers. They were fixed and stored in $10 \%$ formalin. After fixation, their volumes were calculated by water displacement in a graduated cylinder. A plastic container, lined with sponge to decrease sound reflection, was filled with a solution of $7 \%$ glycerol in distilled water, giving a liquid with an acoustic velocity of approximately $1540 \mathrm{~cm} / \mathrm{s}$, which is the assumed velocity of ultrasound in soft-tissue. A wire grid was placed in the bottom of the container to support the prostates, which were angled at $25^{\circ}$ to the vertical, mimicking the normal anatomical alignment of the prostate in the body relative to the position of the transrectal ultrasound transducer.

\subsubsection{D ultrasound imaging technique}

The 3D ultrasound imaging system used here has been described in detail in Chapter 2. An ATL UM9 ultrasound machine with a side-firing, $5 \mathrm{MHz}$ linear array transrectal ultrasound transducer (ATL, Advanced Technology Laboratories, Bothell, Washington) was used for this study.

The probe holder was fixed to a metal stand in a manner that allowed the distal end of the transducer to be immersed in the glycerol solution with its transducer positioned within $2 \mathrm{~cm}$ of the prostate. After allowing the solution to settle, the stepping motor was activated causing the transducer to rotate about 
its long axis. The angle of rotation, typically $100^{\circ}$, depended on the size of the prostate. During this rotation, typically $1002 \mathrm{D}$ ultrasound images were captured by a video frame grabber and stored in the microcomputer. A data acquisition rate of 5 images/sec was used so that for a typical data set of 100 images, the acquisition time was 20 seconds. After the image data acquisition, the 20 image data set was transferred via a local area network from the image acquisition computer (PC) to an image processing computer (Macintosh $840 \mathrm{AV}$ ). The 3D images were then reconstructed using software ported from the reconstruction program described in Chapter 2.

A 3D image processing program called "Slicer" has been developed in our laboratories especially for prostate image display and volume estimation (Fenster et al. 1995). The 3D images were displayed using multiplanar texture mapping technique which displays the 3D image as a multi-sided cube with the appropriate ultrasound image shown on each side of the cube (Robb 1995). The 3D image can be rotated and each of its faces can be moved in and out (i.e. "sliced") to reveal other views of the anatomy. In addition to the standard transaxial and sagittal planes, coronal and oblique planes which are not available with standard $2 D$ ultrasound can be obtained by slicing into the $3 D$ image with oblique planes.

\subsubsection{Accuracy of volume measurements}

For volume estimation, the measurement software requires the operator to select a slice in a particular orientation within the 3D image. In this study, the viewing plane most suited to outlining was usually the transaxial plane. The areas of the 3D ultrasound slices were obtained using the manual outlining method. Inter-slice distances of $2 \mathrm{~mm}$ peripherally in the prostate and of $5 \mathrm{~mm}$ centrally were usually used in this study. Typically, 20 to 30 outlines were obtained on each prostate, depending on the size of the prostate. In some cases, by the nature of the slice angle, the prostate area was fragmented. 
When this occurred, each distinct area was outlined separately, their areas calculated and added together to obtain the total area for that slice.

The prostate volume was then determined by summing the areas and multiplying by the inter-slice distance. This procedure was carried out for the six fixed prostates and the results were correlated with the true volumes, as determined by water displacement, using linear regression analysis. The regression analysis gave the slope of the best-fit line, and the coefficient of determination $r^{2}$, which is a measure of the straight-line relationship (Zar 1984). Also, the accuracy (rms deviation from the line of identity) and the precision (rms deviation from the best fit line) of the measurements were computed. In addition, inverse prediction analysis was also carried out to assess the confidence in predicting the true volumes at a specified confidence level $(95 \%)$ from the results of the linear regression analysis (Zar 1984). This analysis was carried out for volumes of $20,40,60,80$, and $100 \mathrm{~cm}^{3}$ and the results are shown in Table 3.1 in the next section.

\subsubsection{Preparation of pathology slides}

After the prostate specimens were imaged, whole mount sections of the prostates were prepared in a standard manner. Four prostate glands were sectioned in an axial plane, one was sectioned in a coronal plane and one was sectioned in a sagittal plane. Two of the four prostates cut in the axial plane were discarded because some tissue fragmentation occurred during the pathology slide preparation process. The tissue blocks obtained during sectioning were approximately $5 \mathrm{~mm}$ thick. From these, a microscopic slide was prepared and stained with hematoxylin and eosin. One representative slide was obtained from each block. The edge of the prostate in some of the histology slides from the periphery of the remaining prostates were irregular and fragmented in outline and these slides were excluded from further study, as they would lead to inaccurate area measurements. The slides were digitized with a digital video camera, and the digitized image data sets were converted into 
Slicer compatible format, which were then evaluated and measured with the same software used to evaluate the 3D ultrasound images.

\subsubsection{Comparison of 3D US slices and pathology slides}

The 3D ultrasound images of the prostate were examined with Slicer and viewing planes were obtained that corresponded with the planes of the whole mount pathology sections. One 3D image slice, which matched closely in content and contour with a corresponding whole mount section slide, was chosen as the reference slice for that $3 D$ ultrasound image. Slicer then displayed a slice which was parallel to the reference slice, but at a distance of about $5 \mathrm{inm}$ away, as this was the thickness of the prostate section blocks. Once the display was in this location, slight adjustment moving in and out of the 3D image was made in an attempt to identify the 3D ultrasound image slice that most accurately matched with the pathology slide.

The areas of each of the 3D ultrasound slice / pathology slide matched pairs were obtained using the manual outlining method. A computer mouse pointer was used to outline the boundaries of the prostate and seminal vesicles. The software calculated the area within each outlined boundary by counting the pixels within the outline and multiplying that number by the pixel size. The areas for each pair were then computed and compared using linear regression analysis, which gave the best-fit line and the coefficient of determination.

\subsubsection{Precision of area and volume measurements}

Prostate images from digitized pathology slides and slices obtained from the 3D images were repeatedly outlined to assess precision of area measurement. Four prostate images were outlined: two from digitized pathology slides, and two from 3D ultrasound image slices. Each image was repeatedly outlined 20 times. The means and standard deviations of the area measurements were calculated. 
The precision of volume estimation from a $3 D$ image was obtained from 10 repeated volume measurements. The 3D image was outlined in successive transaxial slices, with an inter-slice thickness of $1 \mathrm{~mm}$. The volume was calculated by summing the areas of all the outlined slices, and then multiplying by the inter-slice thickness.

\subsubsection{Inter-slice distance}

As the prostate volume is calculated by summing the outlined areas and multiplying by the inter-slice distance, the choice of inter-slice distances affects the resulting volume estimation. A smaller inter-slice distance produces more accurate volume estimation, but is more time-consuming because more $2 D$ image slices need to be outlined for each volume measurement.

To examine the effect of the choice of the inter-slice distance, the volume of one of the prostates was determined from the 3D image by the outlining method in the transaxial, sagittal, and coronal planes, with inter-plane distance ranging from $1 \mathrm{~mm}$ to $15 \mathrm{~mm}$. Results are presented in Figure 3.3.

\subsection{Results}

\subsubsection{Accuracy of volume measurements}

Figure 3.1 shows a plot of the prostate volumes measured from $3 D$ US vS the true volume determined by water displacement. Also plotted is the-leastsquares fit line, with a slope of $1.006 \pm 0.007$. The coefficient of determination $r^{2}$, which is a measure of the straight-line relationship, was 0.9997 . The accuracy (rms deviation from the line of identity) of the measurements was $2.6 \%$, and the precision (rms deviation from the best fit line) was $2.5 \%$.

\subsubsection{Comparison of 3D US slices vs pathology slides}

Figure 3.2 shows the correlation between areas measured from digitized pathology slides and from their corresponding views from the 3D ultrasound image slices. The solid line is the best fit line by least squares, with a slope of 
$1.086 \pm 0.040$. The coefficient of determination $r^{2}$ was 0.984 . It is seen that the prostate areas obtained from the 3D uitrasound image are overestimated by about $9 \%$, as compared to the areas obtained from the pathology slides.

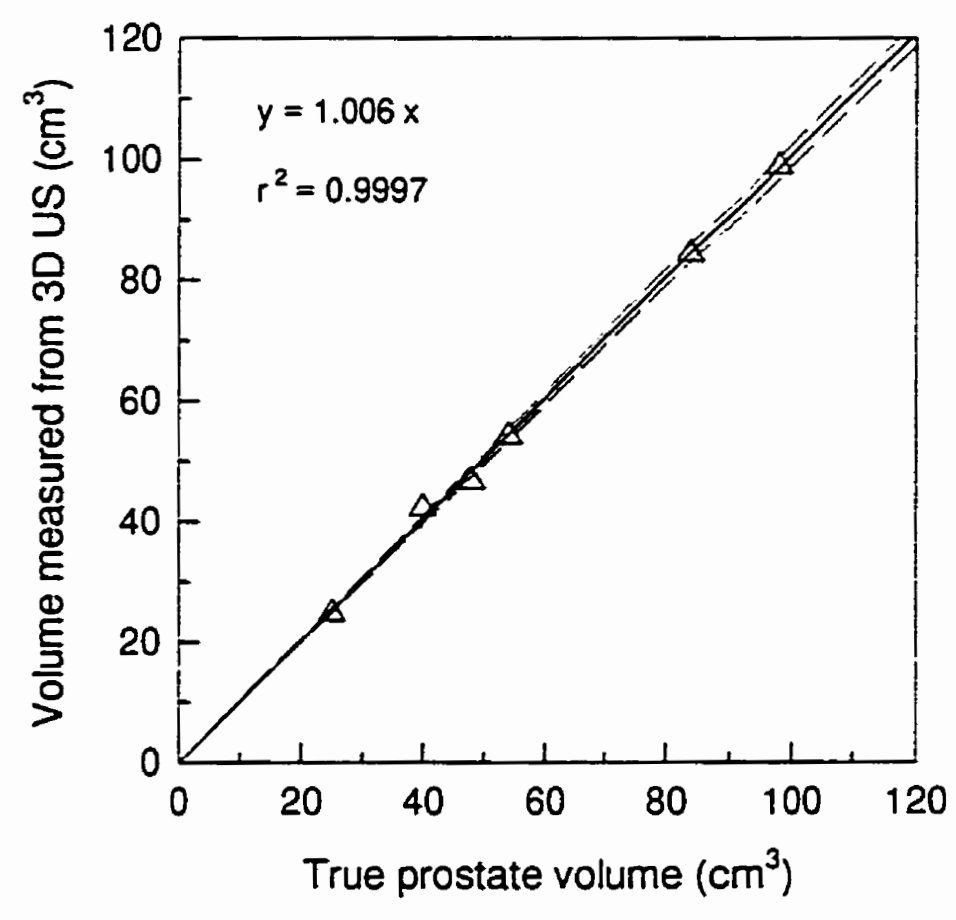

Figure 3.1 The prostate volume measured from the three-dimensional (3D) ultrasound images compared with the volume measured by water displacement. The solid line is the best fit line by least squares with a slope of $1.006 \pm 0.007$. The dashed lines are the $95 \%$ confidence intervals of the regression. 


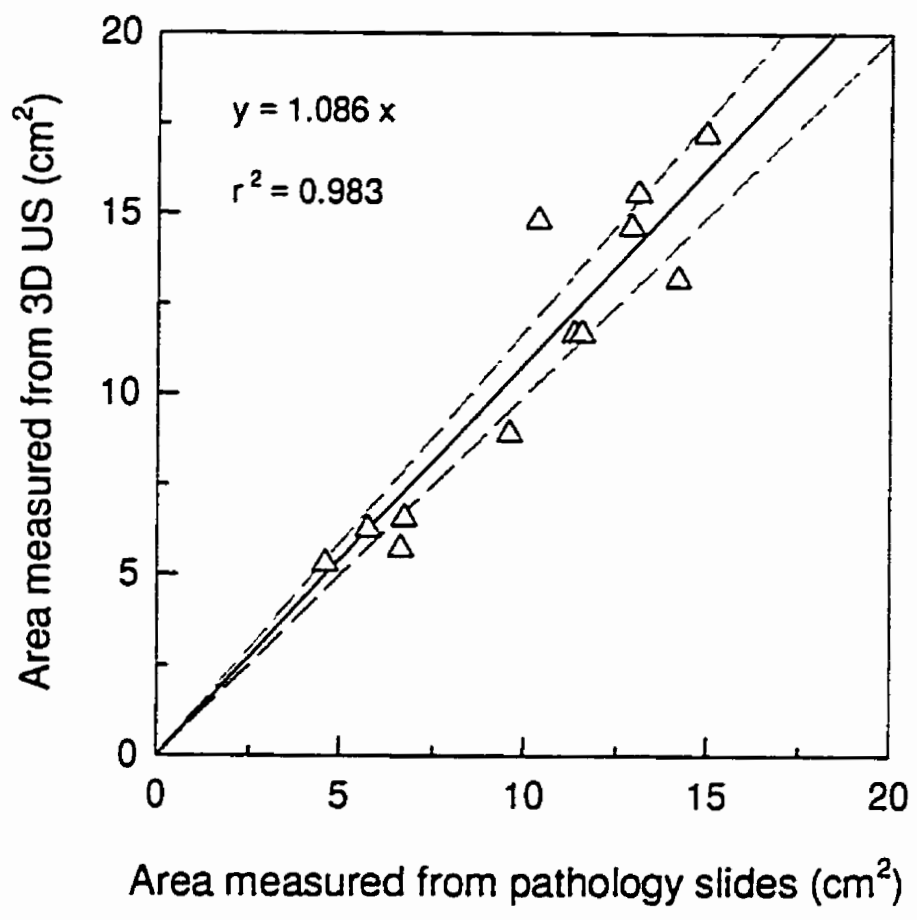

Figure 3.2 Prostate area obtained from digitized pathology slides compared to the area obtained from corresponding views from the 3D ultrasound image slices. The solid line is the best fit line by least squares, with a slope of $1.086 \pm$ 0.040 . The dashed lines are the $95 \%$ confidence intervals of the regression. 
Table 3.1 shows the predicted volumes and their $95 \%$ confidence interval for a range of measured volumes using the $3 \mathrm{D}$ ultrasound technique. From this table it is seen that the $95 \%$ confidence interval ranges from about 6 to $7 \mathrm{~cm}^{3}$.

Table 3.1 Inverse prediction of the true volumes and their $95 \%$ confidence interval for the $3 D$ US measured volumes of $20,40,60,80$, and $100\left(\mathrm{~cm}^{3}\right)$.

\begin{tabular}{ccc}
\hline $\begin{array}{c}\text { 30 US Volume } \\
\left(\mathrm{cm}^{3}\right)\end{array}$ & $\begin{array}{c}\text { Predicted Volume } \\
\left(\mathrm{cm}^{3}\right)\end{array}$ & $\begin{array}{c}\text { Confidence Interval } \\
\left(\mathrm{cm}^{3}\right)\end{array}$ \\
\hline 20 & 19.9 & $16.9-22.8$ \\
40 & 39.8 & $36.7-42.8$ \\
60 & 59.6 & $56.5-62.8$ \\
80 & 79.5 & $76.2-82.8$ \\
100 & 99.4 & $95.9-102.9$ \\
\hline \hline
\end{tabular}

\subsubsection{Precision of area and volume measurements}

Means and standard deviations of area measurements of the prostate images from the 3D ultrasound images and the digitized pathology slides are tabulated in Table 3.2. As can be seen from the table, the standard deviation for area measurements by the manual outlining technique is very small. The precision of area estimation, given by the standard deviation as percentage of the mean, was about $0.8 \%$ for the pathology slides, and about $1.1 \%$ for the slices obtained from the 3D images.

The precision of the volume measurement was obtained from 10 repeated measurements of a 3D image of the prostate. The volume was determined from outlining the $3 D$ image in successive transaxial slices, with an inter-slice distance of $1 \mathrm{~mm}$. The mean of the prostate volume was $25.32 \mathrm{~cm}^{3}$, and the standard deviation was $0.43 \mathrm{~cm}^{3}$, or $1.7 \%$ of the mean. This measurement of the 
precision was used in subsequent experiments as an indication of the uncertainties of volume measurements.

Table 3.2 Precision of outlining an image. The means and standard deviations (S.D.) were obtained by repeated outlining each image 20 times by the same observer.

\begin{tabular}{cccc}
\hline Images & Mean $\left(\mathrm{mm}^{2}\right)$ & S.D. $\left(\mathrm{mm}^{2}\right)$ & S.D. / Mean \\
\hline A: Pathology slide & 436 & 3.1 & $0.71 \%$ \\
B: Pathology slide & 553 & 4.4 & $0.79 \%$ \\
C: 3D US slice & 560 & 6.2 & $1.10 \%$ \\
D: 3D US slice & 574 & 6.3 & $1.10 \%$ \\
\hline
\end{tabular}

\subsubsection{Inter-slice distance}

Figure 3.3 shows the prostate volume obtained from outlining the $3 D$ image in three different viewing planes (transaxial, sagittal, and coronal), for inter-slice distances ranging from $1 \mathrm{~mm}$ to $15 \mathrm{~mm}$. The error bars represent one standard deviation as obtained from the volume measurement precision experiment described in the previous section. From Figure 3.3, it is apparent that measured prostate volume is constant to within the precision of the volume estimation up to an inter-slice distance of $8 \mathrm{~mm}$. Using inter-slice distances greater than $8 \mathrm{~mm}$ results in an underestimation of the prostate volume. 


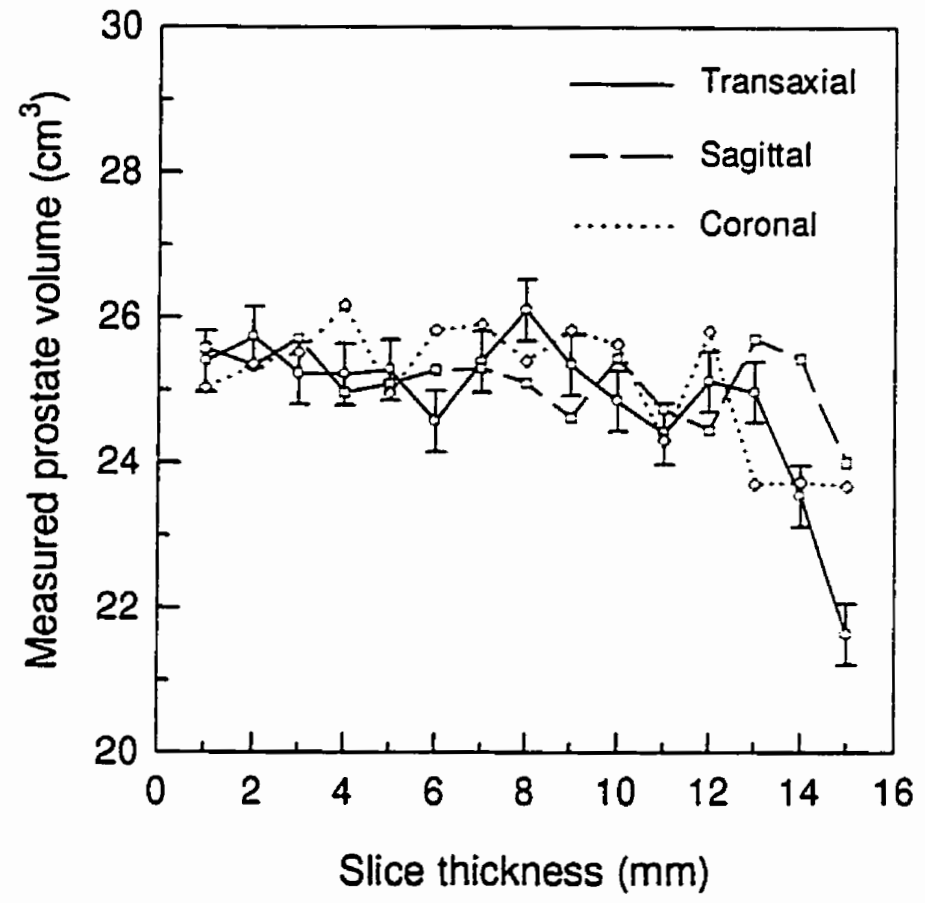

Figure 3.3 The prostate volume measured by outlining a 3D image slice-by-slice for inter-slice distance ranging from 1 to $15 \mathrm{~mm}$. The error bars indicates one standard deviation (1.7\%) of volume estimation by outlining (see section 3.3.3). The slicing of the 3D image was carried out in three viewing planes: transaxial, sagittal, and coronal planes. 


\subsection{Discussion}

In the previous chapter, we have shown that the volume estimation on the laboratory phantoms using the planimetry method with our 3D imaging system was accurate to within $0.9 \%$; while in this chapter, we have shown that in vitro measurements of prostate specimens was accurate to within $2.6 \%$. The slightly decrease in the measurement accuracy is largely due to the fact that it is more difficult to outline accurately images of the prostate than to outline images of the balloon phantoms. The use of formalin-fixed prostates may introduce another potential source of error affecting the measurement results: tissue shrinkage caused by the fixation process (McNeal 1986). Though the speed of sound is not altered in formalin fixed tissues (Yang 1991), formalin alters the normal echogenicity of tissues, making distinction between seminal vesicles and the prostate more difficult on the 3D US. These factors may also account for the errors in the comparison of 3D US slices and pathology slides.

Accurate assessment of prostate volumes are clinically important. Conventionally, the prostate volumes are estimated using the HWL method, i.e., the volumes are calculated from measurement of the height, width, and length of the prostate in two orthogonal $2 \mathrm{D}$ image planes, assuming an ellipsoidal prostate. With 3D ultrasound imaging, the prostate volume can be measured with the planimetry method, i.e., sequential area summation of multiple crosssectional images of the prostate.

Studies have shown that prostate volume estimation using the conventional HWL method has not been very accurate (Littrup et al. 1991; Rahmouni et al. 1992; Terris et al. 1991, 1992). This is largely due to the fact that the HWL method assumes that prostate is a simple ellipsoid, which is not true. The planimetry method removes this assumption, thus it would offer better accuracy. Sehgal et al. (1994) has reported that volume estimation of waterfilled balloons using planimetry method was accurate to within $3 \%$. As a 
comparison, Rahmouni et al. (1992) has shown that the HWL method underestimated prostate volume by $36 \%$.

Although the 3D planimetry method offers a more accurate volume estimation, manually outlining the prostate image slice by slice is tedious and time-consuming. This process can takes 30 to 120 minutes, depending on the size of the prostate and the inter-slice thickness, i.e., the number of crosssectional images to be outlined. One solution to this problem would be to use computer algorithms to perform semi-automated or fully automated prostate image segmentation.

In conclusion, the 3D ultrasound imaging technique for prostate volume estimation offers a high degree of accuracy. 3D ultrasound imaging is likely to be a better tool than standard 2D ultrasound for prostate volume estimation, and therefore would be helpful in assessing a patient's response to a particular intervention.

\subsection{References}

1. Fenster A, Dunne S, Chan TKC, Downey DB. Method and system for constructing and displaying three-dimensional images. The United States Patent 5,454,371 (October 3, 1995).

2. McNeal JE, Kindrachuk RA, Freiha FS, Bostwick DG, Redwine EA, Stamey TA. Patterns of progression in prostate cancer. Lancet 1(8472):60-63; 1986.

3. Kirby RS, Christmas TJ, Brawer M. Prostate Cancer. London: Times Mirror International Publisher Limited; 1996: 77-92 (Chapter 8: Imaging and staging of prostate cancer).

4. Littrup PJ, Williams CR, Egglin TK, Kane RA. Determination of prostate volume with transrectal US for cancer screening: Part 2. Accuracy of in vitro and in vivo techniques. Radiology 179: 49-53; 1991. 
5. Rahmouni A, Yang A, Tempany CM, Frenkel T, Epstein J, Walsh P, Leichner PK, Ricci C, Zerhouni E. Accuracy of in-vivo assessment of prostatic volume by MRI and transrectal ultrasonography. J Comput Assist Tomogr 16: 935-940; 1992.

6. Rankin RN, Fenster A, Downey DB, Munk PL, Levin MF, Vellet AD. Three-dimensional sonographic reconstruction: techniques and diagnostic applications. Am J Roentgenol 161:695-702; 1993.

7. Robb RA. Three Dimensional Biomedical imaging: Principles and Practice. New York: VCH Publishers Inc., 1995.

8. Sehgal CM, Broderick GA, Whittington R, Gorniak RJT, Arger PH. Threedimensional US and Volumetric Assessment of the Prostate. Radiology 192: 274-278; 1994.

9. Terris MK, Stamey TA. Determination of prostate volume by transrectal ultrasound. J Urol 145: 984-987; 1991.

10. Terris MK, MCNeal JE, Stamey TA. Estimation of prostate cancer volume by transrectal ultrasound imaging. J Urol 147:855-857; 1992.

11. Tong S, Downey DB, Cardinal HN, Fenster A. A three-dimensional ultrasound prostate imaging system. Ultrasound Med Biol 22: 735-746; 1996.

12. Wingo PA, Tong T, Bolden S. Cancer Statistics 1995. CA Cancer J Clin 45: 8$30 ; 1995$.

13. Yang JN, Murphy AD, Madson EL, Zagzebski JA, Gilchrist KW, Frank GR, MacDonald MC, Millard CA, Faraggi A, Jaramillo CA: A method of in vitro mapping of ultrasound speed in breast tissue. Ultrason. Imaging 13: 91-109; 1991.

14. Zar JH. Biostatistical analysis. 2nd edition. New Jersey: Prentice Hall Inc, 1984. 


\section{ANALYSIS OF IMAGE DISTORTION}

\subsection{Introduction}

Accurate prostate volume measurement is necessary for the interpretation of serum tests for prostate specific antigen (PSA), which has been increasingly used to screen for prostate cancer (Benson et al. 1992). Thus, an accurate, reproducible technique for measuring prostate and tumor volumes would be an invaluable aid in the early detection and accurate staging of prostate cancer.

Until now, quantitative estimation of these volumes has been obtained by using two-dimensional (2D) transrectal ultrasound (TRUS), although with less accuracy than clinically desirable (Terris et al. 1992). Usually, this is done by measuring the height, width, and length $(H W L)$ from two orthogonal views, and then calculating the volume by assuming an ellipsoidal shape for the prostate, even though this approach could potentially lead to inaccurate results, particularly for tumors with highly non-ellipsoidal shapes. A more accurate method (Terris et al. 1992, Sehgal et al. 1994) is to use planimetry, i.e. to "slice" the volume into a number of parallel cross-sections, whose areas are then multiplied by the interslice spacing and summed, to obtain the prostate or tumor volume. However, this approach is difficult to implement when only a limited number of $2 D$ views are available, as with conventional 2D TRUS.

In order to overcome this difficulty, it has been proposed to use 3D TRUS to obtain a volume image of the prostate, which can then be virtually "sliced" in the computer at any angle, enabling accurate, reproducible planimetry to be performed (Sehgal et al. 1994, Elliot et al. 1996, Tong et al. 1996). This and other computer techniques and clinical applications has been discussed in several review articles, which comprehensively describe the historical background, current state-of-the-art, and future of 3D ultrasound imaging (Fenster and Downey 1996, Rankin et al. 1993, Greenleaf et al. 1993, Belohlavek et al. 1993).

Currently, 3D TRUS images are created by collecting a series of 2D images while the ultrasound probe is linearly withdrawn (Sehgal et al. 1994) or rotated 
(Tong et al. 1996, Elliot et al. 1996). Two types of TRUS probes are suitable for rotational scanning. One is the end-firing probe, where the transducer is at the probe tip, and the probe axis extends through the cone-shaped volume containing the acquired images. Because of this, end-firing probes are very sensitive to errors in the alignment of the beam and rotation axes, since even a small misalignment (such as might be caused by probe movement during the scan, or a systematic error in beam angle, etc.) will result in noticeable artifacts in the reconstructed 3D image. Moreover, the probe must be rotated a full $180^{\circ}$ in order to complete the scan of a 3D volume. The other type is the side-firing probe, where the transducer is mounted parallel to the probe axis, which lies outside the fan-shaped annular volume containing the acquired images. Because of this, 3D image reconstruction is relatively insensitive to an error in the assumed location of the probe axis (as will be shown), and hence to any error in the perpendicularity of the beam and rotation axes. Furthermore, the scanning angle can be less than $180^{\circ}\left(80^{\circ}\right.$ is typical), resulting in finer angular image resolution with the same number of acquired $2 \mathrm{D}$ images.

We have developed a 3D TRUS system for imaging the prostate (Fenster et al. 1996; Tong et al. 1996). We have already shown experimentally that our system yields distance and volume measurements which are both accurate to about $\pm 1 \%$, and that the reconstruction algorithm yields a $3 D$ image resolution that is only negligibly inferior to that of the input $2 D$ images (Tong et al. 1996). In this paper, we theoretically analyze the sources and character of imaging errors in the curvature and length of a straight line, the area of an arbitrary cross-section, or the volume of an arbitrary object, for our system. We then use measurements from simulated images of a cubical object and experimental images of a wire phantom to confirm this analysis numerically and experimentally. Finally, we describe a practical method for reducing these errors to negligible proportions. 


\subsection{Theory}

\subsubsection{Introduction}

Before the ultrasound image data is acquired, a region of interest (ROI) of size $X$ by $Y$ is defined within the full-screen 2D image provided by the ultrasound machine. Data acquisition is then accomplished by rotating the ultrasound probe through an angle $\Theta$ about its central axis, while the frame-grabber digitizes a series of $N 2 D$ images, thereby scanning through a volume which forms an annular sector of a cylinder subtending an angle $\Theta$ about the probe axis, of height $X$ inner radius $R_{0}$, and outer radius $Y+R_{0}$, where $R_{0}$ is the distance from the probe axis to the top edge of the ROI (see Figure 4.1).

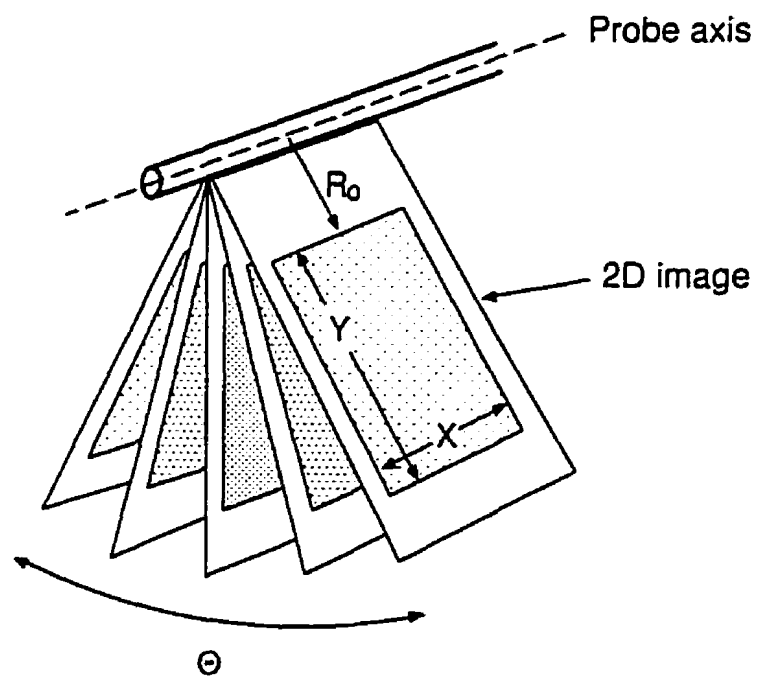

Figure 4.1. A schematic diagram illustrating the geometry used for 3D scanning. A region of interest ( $R O I$ ) is digitized in each of a series of conventional $2 D$ images, which are acquired while the (side-firing) ultrasound probe is rotated about its axis through an angle $\Theta$. The size (in pixels) of the ROI is $X$ (laterally) by $Y$ (axially), and its inner radius is $R_{0}$.

To reconstruct correctly the $3 D$ image from the $2 D$ image data set, the reconstruction algorithm requires that five parameters be accurately known. Of 
these, the first three, the size $X$ by $Y$ of the ROI in each 2D image and the number $N$ of $2 D$ images are known exactly; while the remaining two, the distance $R_{0}$ from the probe axis to the top edge of the ROI, and the total scanning angle $\Theta$, are subject to measurement uncertainties $\Delta R$ and $\Delta \Theta$, which can potentially lead to non-negligible geometric distortion in the reconstructed $3 D$ image. If a stepper motor is used to rotate the probe, $\Delta \Theta$ is (or can be made to be) negligible. However, if a servo motor is used, the transducer is rotated with constant angular velocity for the duration of the image acquisition, which may result in a non-negligible variation $\Delta \Theta$ in $\Theta$. Here, we assume that, to good approximation, the error $\Delta \Theta$ is uniformly distributed over $\Theta$, so that the relative error in any angle is constant, and given by $\Delta \Theta / \Theta$.

Here, we first analyze theoretically the effects that a given error $\Delta R$ or $\Delta \Theta$ has on the $3 D$ image reconstruction. Three kinds of geometric distortions are considered: (i) the relative distortion in the image of a straight line in the object, as measured by the perpendicular deviation of the image from the line joining its endpoints, as a fraction of its length ; (ii) the relative error in the line length; and, (iii) the relative error in the cross-sectional area of an annular sector perpendicular to the probe axis. Since all distances parallel to the probe axis are known exactly, the third error must also apply to a cross-sectional area whose projection is such a sector, or to a volume comprised of a stack of such sectors. Furthermore, since an area of any shape can be tessellated to any required accuracy by a finite set of such sectors, the third error must apply to an arbitrary cross-sectional area, and hence to an arbitrary volume, as well.

To confirm this analysis, simulated images of a cubical object and real images of a wire phantom were used to numerically and experimentally verify that these errors are indeed well described by the simple formulas presented.

Finally, we describe a method to calibrate the system by determining the axis location accurately. Once this is done, it is easy to calibrate the total scanning angle as well. Thus, all of these errors can be virtually eliminated. 


\subsubsection{D image reconstruction}

Cartesian coordinates $\left(x^{*}, y^{*}\right)$ are used in each 2D image of size $(X, Y)$ (Figure 4.1). By convention, the $x^{*}$ and $y^{*}$ directions are chosen respectively as the lateral (parallel to the transducer) and axial (perpendicular to the transducer) directions in each 2D image, so that the probe rotation axis is parallel to the $x^{*}$ axis in each 20 image. (Starred coordinates are used here in order to avoid later confusion with the 3D Cartesian coordinates $(x, y, z)$ introduced below.)

Since the 3D image data set comprises a fan of $2 D$ images centered on the probe axis, it is best described in cylindrical coordinates $(r, \theta, z)$, with the $z$ axis being the probe rotation axis, as shown in Figure 4.2. Each $2 D$ image is then embedded into the $3 \mathrm{D}$ volume via the transformation $\left(y^{*}, \theta, x^{*}\right) \rightarrow(r, \theta, z)$, where $\theta$ is the rotational angle of the probe for that particular 2D image.

The 3D volume image is then reconstructed by remapping the image pixel values on a grid in cylindrical coordinates $(r, \theta, z)$ onto a suitable set of pixel values on a grid in Cartesian coordinates $(x, y, z)$, as shown earlier in Figure 2.1. However, since every 2D image is perpendicular to all the $x-y$ planes of this $3 D$ Cartesian grid, the reconstruction algorithm in each $x-y$ plane, i.e. for every $z$ value, is the same. Thus, for each $z$ value, the pixel value at a Cartesian gridpoint $(x, y)$ is found via bilinear interpolation from those of its four nearest neighbors on the super-imposed grid in polar coordinates $(r, \theta)$, as described by Tong et al. (1996). Moreover, for a given scanning protocol, this process may be made simpler and faster by placing the required interpolation coefficients in a pre-computed look-up table. 


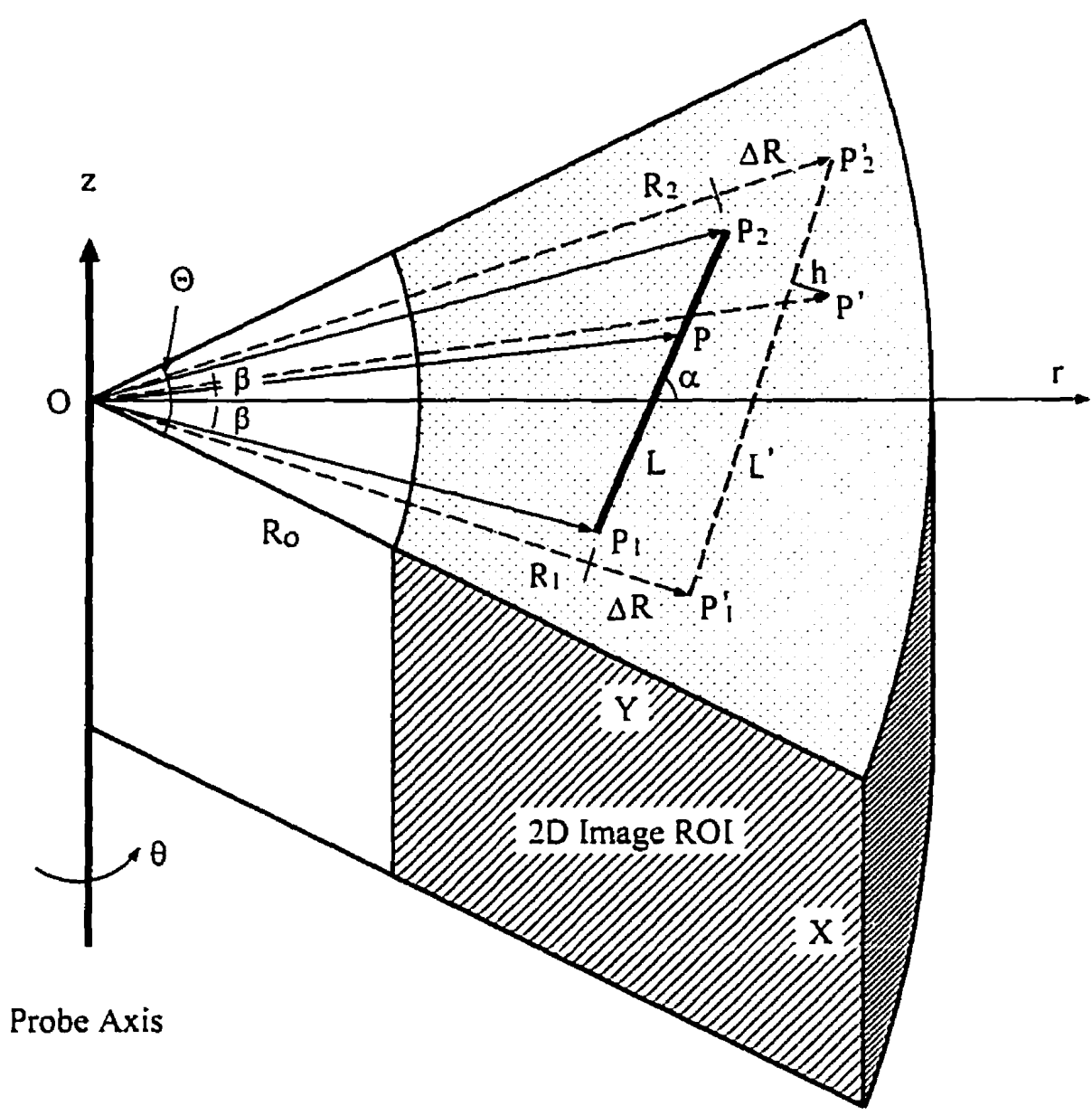

Figure 4.2. Schematic diagram illustrating the variables used to analyze the image distortion of a straight line object. A fan of $2 D$ regions of interest (ROls) of size $X$ by $Y$ pixels is collected while the ultrasound probe is rotated through an angle $\Theta$ about its axis, which forms the $z$ axis. Each $R O I$ is at a distance $R_{0}$ from this axis, and the line $L$ lies in an $r-\theta$ plane, with endpoints $P_{1}=\left(R_{1},-\beta\right)$ and $P_{2}=\left(R_{2}, \beta\right)$ in polar coordinates $(r, \theta)$ in this plane, so that the radial line $\theta=0$ bisects the angle $2 \beta$ subtended by $L$ at the axis. The angle that $L$ makes with $\theta=0$ is denoted by $\alpha$, where $0 \leq \alpha<\pi$ and $0 \leq \beta<\min (\alpha, \pi-\alpha)$. If $R_{0}{ }^{\prime}=R_{0}+\Delta R$ and $\Theta^{\prime}=\Theta+\Delta \Theta$ are (incorrectly) used in the reconstruction algorithm, the endpoints will appear to be at $P_{1}{ }^{\prime}=\left(R_{1}{ }^{\prime},-\beta^{\prime}\right)$ and $P_{2}{ }^{\prime}=\left(R_{2}{ }^{\prime}, \beta^{\prime}\right)$, where $R_{1}{ }^{\prime}=R_{1}+\Delta R, R_{2}{ }^{\prime}=R_{2}+\Delta R$, and $\beta^{\prime}=\beta+\Delta \beta$, with $\Delta \beta / \beta=\Delta \Theta / \Theta$, and the image $L^{\prime}$ of $L$ will not only be distorted in length, compared to $L$, but also in shape, so that the image $P^{\prime}$ of an arbitrary point $P$ on $L$ will be a perpendicular distance $h$ (positive away from the axis) from the straight line joining $P_{1}{ }^{\prime}$ and $P_{2}{ }^{\prime}$. 


\subsubsection{Image distortions}

In the following sections, we describe the effects of an error $\Delta R$ in the probe axis location, or an error $\Delta \Theta$ in the total scanning angle $\Theta$, on the shape and length of the image of a straight line, on the area of the image of an annular sector (and hence of an arbitrary area) and, by extension, on the image of an arbitrary volume. The mathematical derivation is shown in Appendix A.

\subsubsection{Distortion in the image of a straight line}

Figure 4.2 shows the geometry used to analyze the image of a straight line when there is both an error $\Delta R$ in the assumed location of the axis and an error $\Delta \Theta$ in the total scanning angle $\Theta$. A series of 2D images are acquired while the probe is rotated about this axis, and since the distance from the axis to the $\mathrm{ROI}$ in each $2 \mathrm{D}$ image is $R_{0}$, the volume scanned by the probe is an annular sector of inner radius $R_{0}$.

Consider now a line $L$ in an $r-\theta$ plane, whose origin $O$ is the intersection of the axis with this plane. Let $P_{1}$ and $P_{2}$ denote the endpoints of $L$, chosen so that $\theta$ increases in going from $P_{1}$ to $P_{2}$, and define $R_{1}=\left|O P_{1}\right|, R_{2}=\left|O P_{2}\right|$, $R=\left(R_{2}+R_{1}\right) / 2$, and $S=\left(R_{2}-R_{1}\right) / 2$. Also, denote the angle $\angle P_{1} O P_{2}$ by $2 \beta$, and define its bisector as the polar axis $\theta=0$, so that $P_{1}$ and $P_{2}$ have polar coordinates $\left(R_{1},-\beta\right)$ and $\left(R_{2}, \beta\right)$, respectively. Then, as shown in Appendix $A$, the angle $\alpha$ between $L$ and the polar axis satisfies $\tan \alpha=(R / S) \tan \beta$, while the equation of $L$ in polar coordinates can be written as $r \sin (\alpha-\theta)=D$, where $D$ is the perpendicular distance of $L$ from $0,0 \leq \alpha<\pi$, and $|\theta| \leq \beta<\min (\alpha, \pi-\alpha)$.

Now, if the distance $R_{0}$ from the axis to the edge of the ROI is incorrectly estimated as $R_{0}^{\prime}=R_{0}+\Delta R$, then every point in the image is incorrectly remapped outwards $(\Delta R>0)$ or inwards $(\Delta R<0)$ by the same radial distance $|\Delta R|$. Thus, in particular, we have $\Delta R=R^{\prime}-R$, while $\Delta S=S^{\prime}-S=0$.

Similarly, if the total scanning angle $\Theta$ is incorrectly estimated as $\Theta^{\prime}=\Theta+\Delta \Theta$, then, assuming that, to good approximation, the angular interval 
between successive 2D image acquisitions remains uniform throughout the scan, every point in the image is incorrectly remapped away from $(\Delta \Theta>0)$ or towards $(\Delta \Theta<0)$ the polar axis $\theta=0$ by an angle $\Delta \theta$ proportional to its angular coordinate $\theta$. Thus, in particular, $\Delta \beta=\beta^{\prime}-\beta=\beta \Delta \Theta / \Theta$, so that the relative error $\Delta \beta / \beta=\Delta \theta / \theta=\Delta \Theta / \Theta$ is constant.

As a consequence, the image $L^{\prime}$ of $L$ will change both in shape and length. We consider the former effect here, and the latter in the next section. The amount of distortion of a straight line is best described by the perpendicular height $h$ of the triangle $P_{1}^{\prime} P^{\prime} P_{2}^{\prime}$ consisting of a point $P^{\prime}$ on $L^{\prime}$ and its two endpoints $P_{1}^{\prime}$ and $P_{2}^{\prime}$. Thus, as $\theta$ increases from $-\beta$ to $\beta$, the pre-image point $P$ traverses $L$ from $P_{1}$ to $P_{2}$ as $P^{\prime}$ traverses $L^{\prime}$ from $P_{1}{ }^{\prime}$ to $P_{2}$. Hence, $h=0$ when $\theta= \pm \beta$ or when $P^{\prime}$ is collinear with $P_{1}{ }^{\prime}$ and $P_{2}{ }^{\prime}$, and $h>0$ if the apex of the triangle points away from the axis, in the positive $r$ direction. Also, by definition, the sagitta $s$ of $L^{\prime}$ is the maximum magnitude of $h$.

In Appendix A, we show that $h(\theta)$ is, to good approximation, linear in both $\Delta R$ and $\Delta \Theta$, so that it can be written in the form:

$$
\frac{h}{\ell}=F(\alpha, \beta, \theta) \frac{\Delta R}{R}+G(\alpha, \beta, \theta) \beta \sin ^{2} \alpha \frac{\Delta \Theta}{\Theta},
$$

where $\ell$ is the length of $L$, and

$$
\begin{aligned}
& F(\alpha, \beta, \theta)=\frac{1}{2} \frac{\sin \alpha}{\sin \beta}\left[\frac{\sin (\alpha+\beta) \sin (\alpha-\beta) \cos \theta}{\sin (\alpha-\theta) \cos \beta}+\sin (\alpha-\theta)-\sin (\alpha+\beta)-\sin (\alpha-\beta)\right], \\
& G(\alpha, \beta, \theta)=\frac{\sin (\alpha+\beta) \sin (\alpha-\beta)}{\sin ^{2} \alpha \sin ^{2} 2 \beta \sin (\alpha-\theta)}\left[\sin (\alpha+\theta)-\cos 2 \beta \sin (\alpha-\theta)-\frac{\theta}{\beta} \sin 2 \beta \cos (\alpha-\theta)\right]
\end{aligned}
$$

The functions $F(\alpha, \beta, \theta)$ and $G(\alpha, \beta, \theta)$ are plotted in Figure 4.3 and Figure 4.4, respectively, for selected values of $\alpha$ and $\beta$. From these graphs, it is clear that $|F(\alpha, \beta, \theta)|<1 / 2$ and $0 \leq G(\alpha, \beta, \theta) \leq 1 / 2$. Thus, the relative sagitta $s / \ell$ of $L^{\prime}$ is bounded so that

$$
\frac{s}{\ell}<\frac{1}{2}\left|\frac{\Delta R}{R}\right|+\frac{\beta \sin ^{2} \alpha}{2}\left|\frac{\Delta \Theta}{\Theta}\right|
$$



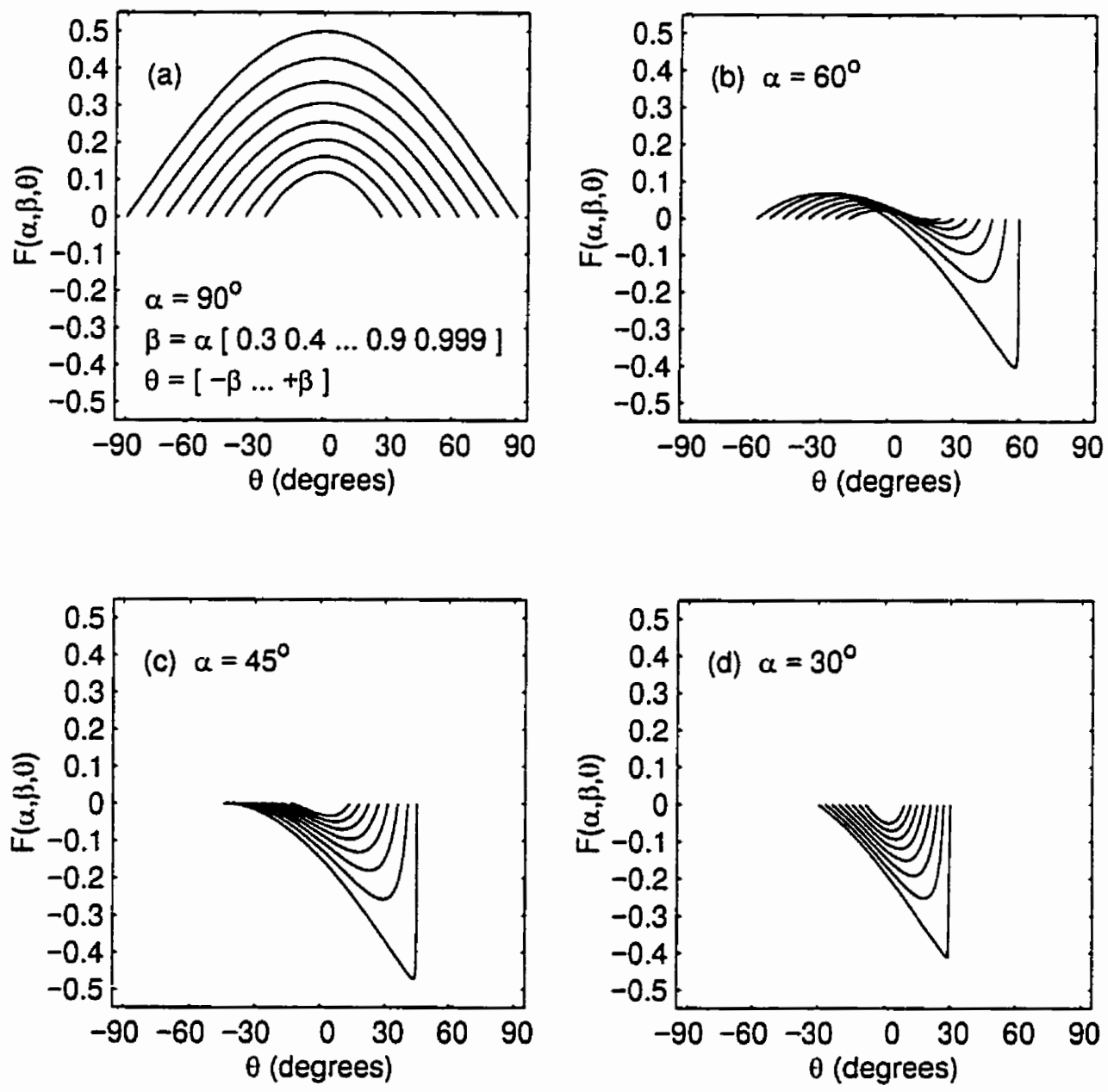

Figure 4.3. Graphs of the function $F(\alpha, \beta, \theta)$ appearing in Eqs. (4.1) and (4.2), plotted as functions of $\theta$ for (a) $\alpha=\pi / 2$, (b) $\alpha=\pi / 3$, (c) $\alpha=\pi / 4$, and (d) $\alpha=\pi / 6$, with $\beta=0.3 \alpha, 0.4 \alpha, 0.5 \alpha, 0.6 \alpha, 0.7 \alpha, 0.8 \alpha, 0.9 \alpha$, and $0.999 \alpha$ in each case. Note that $\max |F(\alpha, \beta, \theta)|$ increases with $\beta$, and $\max |F(\alpha, \beta, \theta)|<1 / 2$ in all cases. 

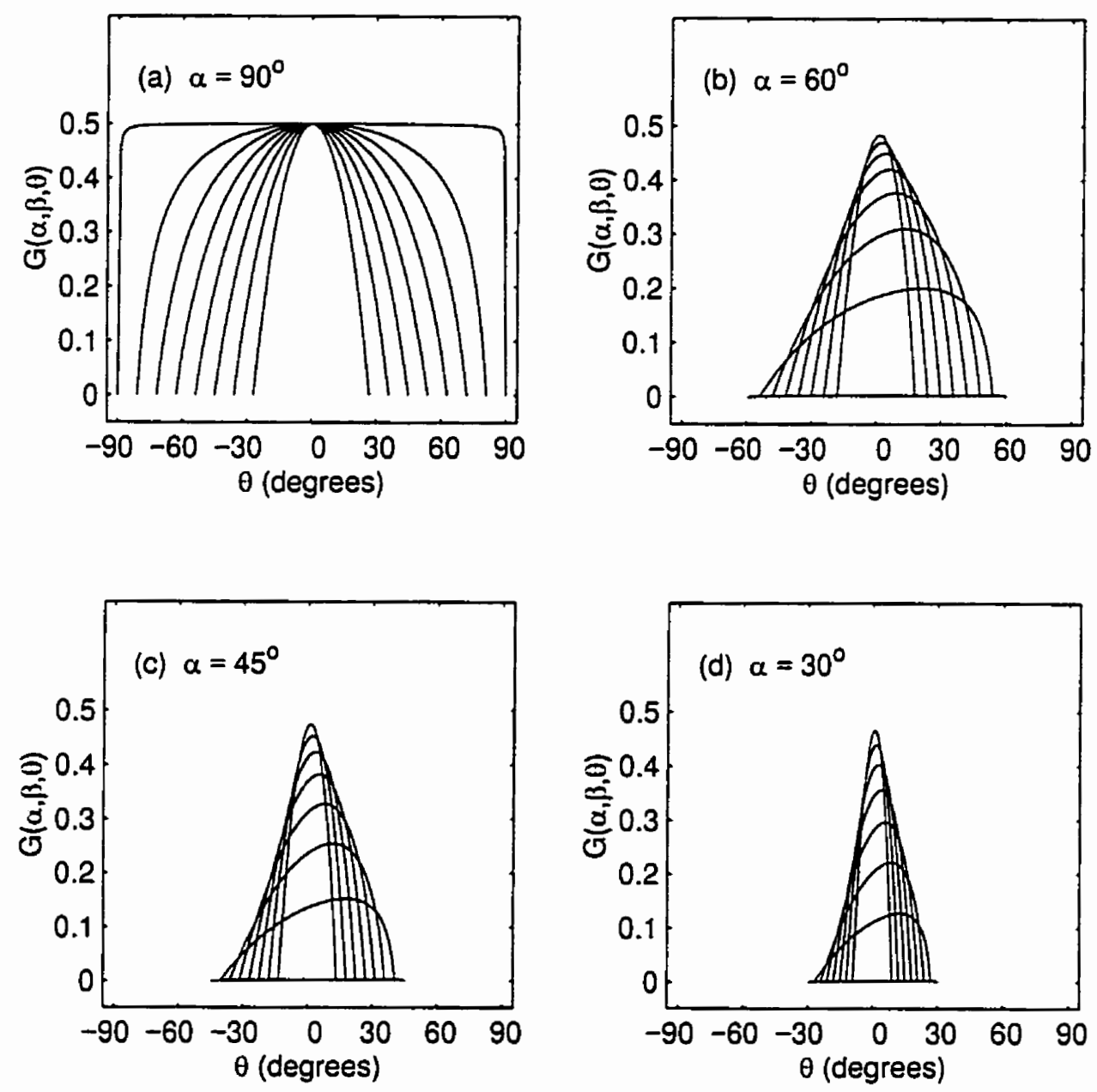

Figure 4.4. Graphs of the function $G(\alpha, \beta, \theta)$ appearing in Eqs. (4.1) and (4.3), plotted as functions of $\theta$ for (a) $\alpha=\pi / 2$, (b) $\alpha=\pi / 3$, (c) $\alpha=\pi / 4$, and (d) $\alpha=\pi / 6$, with $\beta=0.3 \alpha, 0.4 \alpha, 0.5 \alpha, 0.6 \alpha, 0.7 \alpha, 0.8 \alpha, 0.9 \alpha$, and $0.999 \alpha$ in each case. Note that $\max |G(\alpha, \beta, \theta)|$ increases with $\beta$, and $\max |G(\alpha, \beta, \theta)| \leq 1 / 2$ in all cases. 


\subsubsection{Error in length}

Also due to the aforementioned errors, the length $e^{\prime}$ of the line $L^{\prime}$ will differ by an amount $\Delta \ell$ from the length $\ell$ of the line $L$. In Appendix $A$, we show that, correct to first order in $\Delta R / R$ and $\Delta \Theta / \Theta$,

$$
\frac{\Delta \ell}{\ell}=\sin ^{2} \alpha\left[\frac{\Delta R}{R}+H(\alpha, \beta) \frac{\Delta \Theta}{\Theta}\right]
$$

where

$$
H(\alpha, \beta)=\frac{\beta \sin (\alpha+\beta) \sin (\alpha-\beta)}{\sin ^{2} \alpha \sin \beta \cos \beta}=\frac{\beta}{\tan \beta}\left[\frac{1-\frac{\sin ^{2} \beta}{\sin ^{2} \alpha}}{1-\sin ^{2} \beta}\right] .
$$

Furthermore, since $\sin ^{2} \beta<\sin ^{2} \alpha \leq 1$ and $0 \leq \beta<\pi / 2$,

$$
0<H(\alpha, \beta) \leq H(\pi / 2, \beta)=\frac{\beta}{\tan \beta} \leq 1,
$$

with $\beta / \tan \beta=1$ in the limit $\beta \rightarrow 0$. Thus, $\Delta \ell / \ell$ vanishes for a radial line, for which $\alpha=0$, and is maximal for a line perpendicular to a radial line, for which $\alpha=\pi / 2$. Moreover, in this latter case, combining Eqs. (4.5) $-(4.7)$ yields

$$
\frac{\Delta \ell}{\ell}=\frac{\Delta R}{R}+\frac{\beta}{\tan \beta} \frac{\Delta \Theta}{\Theta} \text {. }
$$

\subsubsection{Error in area}

In Appendix $\mathrm{A}$, we show that, for an arbitrary cross-sectional area $A$, the relative error $\triangle A / A$ is exactly

$$
\frac{\Delta A}{A}=\left(1+\frac{\Delta R}{R}\right)\left(1+\frac{\Delta \Theta}{\Theta}\right)-1=\frac{\Delta R}{R}+\frac{\Delta \Theta}{\Theta}+\frac{\Delta R}{R} \frac{\Delta \Theta}{\Theta} .
$$

provided that the average radius $R$ of the area is defined as 


$$
R=\frac{\iint_{A} r d r d \theta}{\iint_{A} d r d \theta}=\frac{A}{A_{0}},
$$

where $A$ is the reconstructed (destination) image area and $A_{0}$ is the source (unreconstructed) image area. The area $A$ can be obtained by simply counting the number of object pixels in the reconstructed image. However, the number of pixels in the corresponding area $A_{0}$ in the source image must be scaled by a factor $K$ equal to the scanning angle (in radians) per pixel. This factor may be conveniently calculated by dividing the total scanning angle $\Theta$ by the total number of spaces between $2 \mathrm{D}$ planes in the source image (see Figure 2.2), which is one less than the total number of $2 \mathrm{D}$ image planes.

\subsubsection{Error in volume}

A 3D volume image is formed by generating an area image in Cartesian coordinates $(x, y)$ for each value of $z$, and repeating as necessary. Since the spacing between successive $z$ values (image slices) is precisely known, the relative error $\Delta V / V$ in an arbitrary volume $V$ is the same as the relative error $\Delta A / A$ in an arbitrary area, namely

$$
\frac{\Delta V}{V}=\frac{\Delta R}{R}+\frac{\Delta \Theta}{\Theta}+\frac{\Delta R}{R} \frac{\Delta \Theta}{\Theta},
$$

which is again exact, provided the average radius $R$ of the object is now given by

$$
R=\frac{\iiint_{V} r d r d \theta d z}{\iiint_{V} d r d \theta d z}=\frac{V}{V_{0}},
$$

where $V$ is the reconstructed image volume and $V_{0}$ is the unreconstructed image volume, in precise analogy to the previous result. 


\subsection{Methods}

\subsubsection{Computer simulation of errors}

A computer simulation was performed to verify the above theoretical analysis. First, a computer algorithm generated a sequence of $2 D$ images, simulating a 3D ultrasound scan of a white cube set in a gray background, with a total scan angle $\Theta$ of $60^{\circ}$. Thus, each $x-y$ cross-section of the 3D image is an annular sector of inner radius $R_{0}=100$ pixels and outer radius $R_{0}+Y=300$ pixels, containing a white square of area $A=120 \times 120$ pixels $^{2}$, centered on polar coordinates $(r, \theta)=(200$ pixels, 0$)$, as shown schematically in Figure 4.5.

Since image distortions arise from two sources of error, $\Delta R$ and $\Delta \Theta$, a series of $3 D$ reconstructions of the cubical object were performed by using different values of $R_{0}^{\prime}=R_{0}+\Delta R$, corresponding to $\Delta R=0, \pm 10, \pm 20$, or \pm 30 pixels, with $\Delta \Theta=0$, and different values of $\Theta^{\prime}=\Theta+\Delta \Theta$, corresponding to $\Delta \Theta=$ $0, \pm 5^{\circ}, \pm 10^{\circ}$, or $\pm 15^{\circ}$, with $\Delta R=0$, to cover a wide range of distortions.

When the correct values of $R_{0}$ and $\Theta$ is used in the reconstruction, so that $\Delta R=\Delta \Theta=0$, the $x-y$ cross-section shown in Figure 4.5 contains a white square, as expected. When $\Delta R$ or $\Delta \Theta$ is non-zero, the reconstructed image is distorted. In each case, the amount of distortion in the length $L^{\prime}$ of the side of the distorted square closest to the axis and in the distorted square's area $A^{\prime}$ were measured from the reconstructed image, and compared to the amounts predicted by our simple theoretical formulas. Also, since every $x-y$ cross-section is the same, and the image height in the $z$-direction is independent of $\Delta R$ and $\Delta \Theta$, the relative error in cube volume $V$ will be identical to that in $A$. 


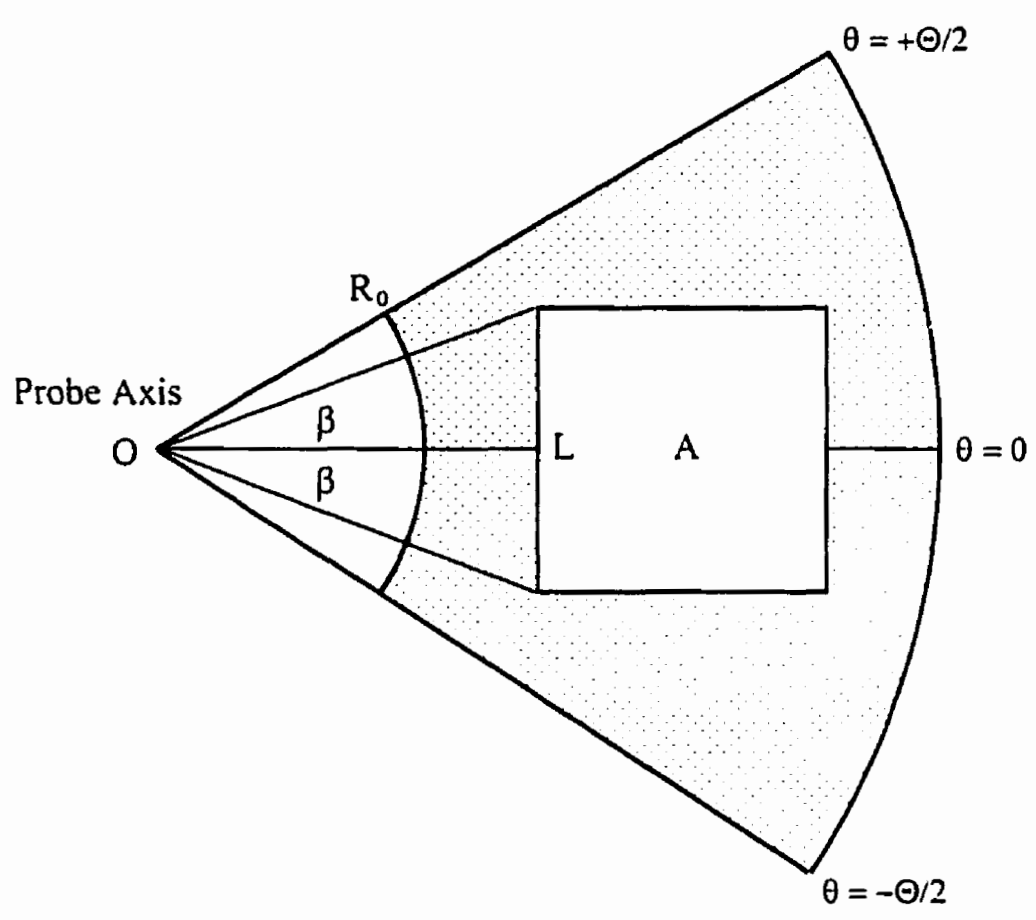

Figure 4.5. Schematic diagram illustrating an $x-y$ cross-section of the 3D image of a white cube set in a gray background. The $z$, or rotation, axis coincides with the probe axis, and comes out of the page at the origin $O$ of polar coordinates. The cube cross-section is a white square symmetric about the positive $y$ axis, which is the line $\theta=0$, so that the angle $\alpha$ between the side $L$ and this line is $\alpha=\pi / 2$ here. In our simulation, the square has an area $A=120 \times 120$ pixels ${ }^{2}$, centered on polar coordinates $(r, \theta)=(200$ pixels, 0$)$, so that the semi-angle $\beta$ subtended by $L$ at $O$ is $\beta=\arctan (60 / 140) \equiv 0.4049$ here. The inner and outer radii of the gray background sector are $R_{0}=100$ pixels and $R_{0}+Y=300$ pixels, respectively, and the total scanning angle is $\Theta=\pi / 3$.

In Appendix $B$, we show that if the image of a sharp edge has a finite width of $n$ pixels, and the probability of manually positioning a cursor on any one of these pixels is the same, then the variance $\sigma^{2}$ in the measured location of the edge is $\sigma^{2}=\left(n^{2}-1\right) / 12$ pixels $s^{2}$. For example, for $n=4$ pixels, the standard deviation $\sigma$ of an edge position is 1.25 pixels, and the corresponding error in measuring the length of a line is $\sqrt{ } 2 \sigma$, or about 1.77 pixels. Thus, since the amount of perpendicular distortion $h$ in the image of $L$ is only a couple of pixels, 
no comparison between measured and predicted amounts of $h$ was attempted.

\subsubsection{Error in length}

In each case, the length $\ell^{\prime}$ of $L^{\prime}$ was measured as the distance between its endpoints, which were each located by manually positioning a cursor on the reconstructed image. The amount of distortion $\Delta \ell=\ell^{\prime}-\ell$, was then found by comparing $\ell \cdot$ to its undistorted value $\ell$ of 120 pixels. Since $\alpha=90^{\circ}$ here, the theoretical relative error $\Delta \ell / \ell$ is given by Eq. (4.8), where $R$, the average distance from the axis to the endpoints of $L$, is $R=\sqrt{ }\left(60^{2}+140^{2}\right)=152.3$ pixels here, and $\beta$, the semi-angle subtended by $L$ at the axis, is $\beta=\arctan (60 / 140)=$ $0.4049 \mathrm{rad}\left(23.20^{\circ}\right)$ here, so that $\beta / \tan \beta=0.945$.

\subsubsection{Error in area}

In each case, the area $A^{\prime}$ of the distorted cross section was measured by using software to count the number of pixels greater than or equal to a suitable threshold, and the error $\Delta A=A^{\prime}-A$ calculated by comparison with the theoretical value of $A=120 \times 120$ pixels $^{2}=14400$ pixels $^{2}$. Before 3D image reconstruction, the pixel values were 200 within the white cube, and 100 in the gray background (on an 8-bit scale of 0 to 255). However, due to the bilinear interpolation performed by the $3 \mathrm{D}$ image reconstruction algorithm, edge pixels in the reconstructed cube image have a distribution of pixel values between 101 and 199. The median of this distribution for the undistorted reconstructed image, 169, was used as the threshold value.

Because every $x-y$ cross-section is the same, the average radius $R$ of the cube volume, given by Eq. (4.12), is identical to its $x-y$ cross-sectional area, given by Eq. (4.10). Using the threshold technique, the measured area $A$ of this cross-section in the undistorted reconstructed image is $A=14480$ pixeis $^{2}$, which is close to the theoretical value of 14400 pixels $^{2}$. Again using the threshold technique, we found that the source image of the cube had $N=34833$ pixels ${ }^{2}$. Now, since the source image size in the $\theta$ direction is $\mathbf{5 0 0}$ pixels, and the total 
scanning angle $\Theta=\pi / 3$ radians $\left(60^{\circ}\right)$, the scale factor $K$ of the source image is here $K=\pi / 3 \mathrm{rad} / 499$ pixels $=2.0986 \mathrm{mrad} /$ pixel. Hence the cross-sectional area in the source image is $A_{0}=N K=73.10$ pixels. Thus, by Eq. (4.10), $R=A / A_{0}=198.1$ pixels, which differs by less than $1 \%$ from the radius of the cube's geometric center, 200 pixels.

\subsubsection{Experimental measurement of errors}

A wire phantom (Figure 2.4) was also imaged to verify our theoretical analysis. This phantom, designed and built in our laboratories, contains 32 parallel wires, arranged in four layers of eight wires each. The distance between layers is $1.000 \pm 0.005 \mathrm{~cm}$, as is the separation of wires within each layer.

The phantom was immersed in a bath of $7 \%$ glycerol in distilled water, and imaged so that all the $2 D$ imaging planes were parallel to the wires. Scanning through a total scanning angle $\Theta$ of $60.14^{\circ}, 1002 D$ images were acquired, for a source image scale factor $K=0.6075 \%$ pixel $(10.603 \mathrm{mrad} / \mathrm{pixel}$ ). We selected an ROI size of $X \times Y=145 \times 172$ pixels $^{2}$ and ROI location of $Y_{0}=109$ pixels from the top of each $2 D$ image. At the field-of-view (FOV) setting of $9 \mathrm{~cm}$ used here, the axis was found to be a distance of $C=27.0$ pixels from the top of each $2 \mathrm{D}$ image (by the method described below), so that the axis-toROI distance is $R_{0}=Y_{0}-C=82.0$ pixels in each 2D image at this FOV setting.

Two series of 3D reconstructions of the wire phantom image were performed, to assess image distortions both qualitatively and quantitatively. In the first series, values of $R_{0}^{\prime}=50,60,70,82,90,100$, or 110 pixels were used with $\Theta^{\prime}=60.14^{\circ}(\Delta \Theta=0)$, and values $\Theta^{\prime}=45^{\circ}, 50^{\circ}, 55^{\circ}, 60.14^{\circ}, 65^{\circ}, 70^{\circ}$, or $75^{\circ}$ were used with $R_{0}^{\prime}=82.0$ pixels $(\Delta R=0)$, to generate a series of images in which the qualitative nature of the image distortions could be readily discerned. This is similar to the protocol used with the simulated images. However, these values of $\Delta R$ and $\Delta \Theta$ are much larger than would realistically occur in practice. Therefore, a second series of 3D image reconstructions was 
performed, using $\Delta R=0, \pm 2, \ldots, \pm 10$ pixels with $\Delta \Theta=0$, and $\Delta \Theta=0, \pm 1^{\circ}, \ldots$, $\pm 5^{\circ}$ with $\Delta R=0$, to quantitatively compare experiment to theory for a realistic range of $R_{0}{ }^{\prime}$ and $\Theta^{\prime}$. This comparison was carried out for straight line distortion as well as the errors in length and area previously considered.

\subsubsection{Distortion in the image of a straight line}

The relative distortion $h / \ell$ of a straight line was measured from the image centroids of three collinear wires in the phantom, which describe a line perpendicular to the radial line $\theta=0$, so that $\alpha=90^{\circ}$. By symmetry, $h / \ell$ is theoretically maximal at the center of this line, where $\theta=0$, and thus

$$
\frac{h}{\ell}=\frac{\tan (\beta / 2)}{2} \frac{\Delta R}{R}+\frac{\beta}{2} \frac{\Delta \Theta}{\Theta} \text {. }
$$

by virtue of Eqs. (4.1)-(4.3), and the relative sagitta $s / \ell$ is thus the absolute value of the right-hand-side. Here, the average radius $R$ of the line endpoints, i.e. the average distance from the probe axis to the centroids of the outer two wires in the undistorted reconstructed image, was found to be $R=172.8$ pixels, while the angular separation $2 \beta$ of the two endpoints was calculated as their separation (in pixels) in the source image, multiplied by the source image scale factor $K$, yielding $2 \beta=25.82^{\circ}$ and hence $\beta=12.91^{\circ}(0.2253 \mathrm{rad})$.

Since $\alpha=90^{\circ}, y_{2}^{\prime}-y_{1}^{\prime} \equiv 0$ for the endpoint centroids in the reconstructed image, so that $\ell \cong \ell^{\prime} \cong x_{2}^{\prime}-x_{1}{ }^{\prime}$; hence, the relative distortion was calculated from the distorted centroid location $\left(x_{0}^{\prime}, y_{0}{ }^{\prime}\right)$ of the middle wire image as

$$
\frac{h}{i}=\frac{y_{0}^{\prime}-\left(y_{2}^{\prime}+y_{i}^{\prime}\right) 2}{x_{2}^{\prime}-x_{i}^{\prime}}
$$

from a selected $x-y$ image cross-section. Moreover, assuming that the $y_{n}^{\prime}$ are measured independently, each with a variance $\sigma_{y}^{2}$, we show in Appendix $C$ that the variance in $h / \ell$ is well-approximated by

$$
\sigma_{0}^{2}=2 \frac{\sigma_{y}^{2}}{\ell^{2}}
$$


since the numerator of Eq. (4.14) is much less than the denominator.

\subsubsection{Error in length}

In each case, the distance $\ell^{\prime}$ between the centroids of the distorted endpoint wire images was measured, and the experimental relative error $\Delta \rho / \ell=$ $\left(\ell^{\prime}-\ell\right) / \ell$ calculated, using the distance $\ell=77.8$ pixels corresponding to the true wire separation of $20 \mathrm{~mm}$ in the undistorted $9-\mathrm{cm}$ FOV image. Since $\alpha=90^{\circ}$, the theoretical relative error is again given by Eq. (4.8), with $R=172.8$ pixels and $\beta=12.91^{\circ}(0.2253 \mathrm{rad})$, as found above, so that $\beta / \tan \beta=0.983$ here.

Moreover, assuming that $\varphi^{\prime}=x_{2}^{\prime}-x_{1}^{\prime}$, and that $x_{2}^{\prime}$ are $x_{1}^{\prime}$ are measured independently, each with a variance $\sigma_{x}^{2}$, we show in Appendix $C$ that the variance in $\Delta \ell^{\prime} l \ell$ is well-approximated by

$$
\sigma_{1}^{2}=2 \frac{\sigma_{x}^{2}}{\ell^{2}}
$$

\subsubsection{Error in area}

The image centroids $\left(x_{n}{ }^{\prime}, y_{n}{ }^{\prime}\right)$ (numbered counterclockwise) of three rows of three wires forming a parallelogram of $20-\mathrm{mm}$ base and $20-\mathrm{mm}$ height in the wire phantom were measured. In each case, the area $A^{\prime}$ of the eight-sided polygon image was then calculated from the exterior eight centroids via the formula (generalized from Spiegel, 1968):

$$
A^{\prime}=\frac{1}{2}\left(\left|\begin{array}{ll}
x_{1}^{\prime} & y_{1}^{\prime} \\
x_{2}^{\prime} & y_{2}^{\prime}
\end{array}\right|+\left|\begin{array}{ll}
x_{2}^{\prime} & y_{2}^{\prime} \\
x_{3}^{\prime} & y_{3}^{\prime}
\end{array}\right|+\cdots+\left|\begin{array}{cc}
x_{8}^{\prime} & y_{8}^{\prime} \\
x_{1}^{\prime} & y_{1}^{\prime}
\end{array}\right|\right)
$$

and the relative error $\Delta A^{\prime} / A=\left(A^{\prime}-A\right) / A$ calculated, using the area $A=77.8^{2}$ pixels ${ }^{2}$ corresponding to the true area $A=400 \mathrm{~mm}^{2}$ in the undistorted $9-\mathrm{cm}$ FOV image. Moreover, by assuming that the $x_{n}^{\prime}$ and $y_{n}^{\prime}$ are all measured independently, with a variance $\sigma_{x}^{2}$ in each $x_{n}^{\prime}$ and $\sigma_{y}^{2}$ in each $y_{n}^{\prime}$, and that the polygon deviates only slightly from a square, we also show in Appendix $C$ that 
the variance in $\triangle A^{\prime} / A$ is well-approximated by

$$
\sigma_{2}^{2}=\frac{3}{4} \frac{\left(\sigma_{x}^{2}+\sigma_{y}^{2}\right)}{A}
$$

\subsubsection{Determination of axis location}

In order to determine the axis location, a simple calibration phantom was designed and built. This phantom consists of a square acrylic tube, $210 \mathrm{~mm}$ long, with one side serrated into four $30-\mathrm{mm}$ wide teeth by milling three $30-\mathrm{mm}$ wide slots across it, as illustrated schematically in Figure 4.6. The separation of adjacent edges is $D_{\mathrm{mm}}=30.000 \pm 0.025 \mathrm{~mm}$. Each tooth is then banded by a segment cut from the finger of a latex glove (similar to a thin rubber band) to form a set of eight nearly equidistant parallel latex planes.

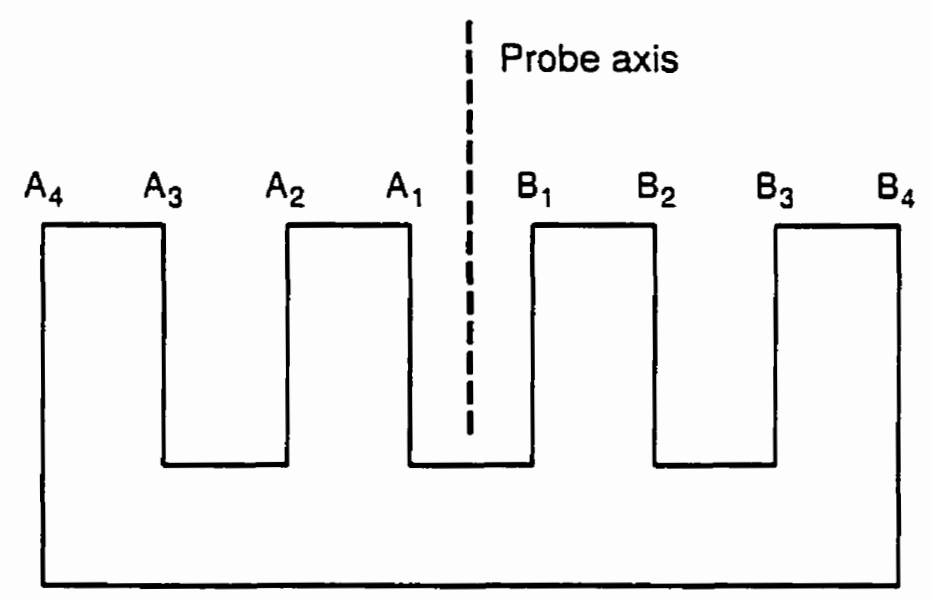

Figure 4.6. Schematic cross-section of the calibration phantom. The phantom consists of a square acrylic tube, serrated on one side into four teeth by three milled slots, forming a series of equally spaced edges, $30.000 \pm 0.025 \mathrm{~mm}$ apart. The teeth are then banded by sections cut from the fingers of a latex glove, and the probe parallelly inserted into the middle slot. The latex planes $A_{1} \ldots A_{4}$ are imaged with the transducer facing to the left, and the planes $B_{1} \ldots B_{4}$ then imaged with the transducer facing to the right, i.e. after rotating the probe $180^{\circ}$. 
The following calibration procedure was then used to determine the axis location: First, the probe is placed in the middle slot and carefully oriented so that its axis is parallel to the latex planes. Then, for each FOV setting, the planes on one side are imaged, the probe is rotated $180^{\circ}$ about its axis, and the planes on the other side are imaged. For our machine, each calibration image pair then contains between two and four parallel lines per image, depending on the FOV.

An image analysis is then performed to determine, first, the physical thickness $d_{\mathrm{mm}}$ of a latex plane; second, the absolute pixel size $p_{\mathrm{mm}}$ for each FOV; third, the distance $C$ (in pixels) of the axis from the top of the full-screen $2 D$ image for each FOV; and finally, a method for checking the consistency of these values, and determining the experimental uncertainty in $C$ for any FOV.

\subsubsection{Latex plane thickness}

At a given FOV setting, let $A_{n}$ denote the locations of the line centroids in the first image, and $B_{n}$ those in the second, measured in pixels from the top of each full-screen 2D image, and numbered as shown in Figure 4.6. Then, for an image pair containing at least three lines per image, the analysis of Appendix $D$ shows that the latex plane thickness $d_{\mathrm{mm}}$ can be found from $D_{\mathrm{mm}}$ by the formula

$$
d_{m m}=D_{m m} \frac{2\left(A_{2}+B_{2}\right)-\left(A_{3}+B_{3}\right)-\left(A_{1}+B_{1}\right)}{\left(A_{3}+B_{3}\right)-\left(A_{1}+B_{1}\right)} .
$$

This calculation was performed at all suitable FOV settings, and the results averaged to yield a more accurate value of $d_{\mathrm{mm}}$.

\subsubsection{Pixel size}

Once $D_{\mathrm{mm}}$ and $d_{\mathrm{mm}}$ are known, only two lines per image are needed to calculate the pixel size $p_{\mathrm{mm}}$, a requirement met by all our image pairs. The analysis of Appendix $D$ then shows that $p_{m m}$ is given at each FOV setting by

$$
p_{m m}=\frac{2\left(D_{m m}+d_{m m}\right)}{\left(A_{2}+B_{2}\right)-\left(A_{1}+B_{1}\right)}
$$




\subsubsection{Axis location}

Finally, given $D_{\mathrm{mm}}, d_{\mathrm{mm}}$, and $p_{\mathrm{mm}}$, the analysis of Appendix $\mathrm{D}$ shows that the axis is location $C$, measured in pixels from the top of the full-screen $2 D$ image, is given for each FOV setting by

$$
C=\frac{\left(A_{1}+B_{1}\right)}{2}-\frac{\left(D_{m m}-d_{m m}\right)}{2 p_{m m}} \text {. }
$$

\subsubsection{Check plot and experimental error in axis location}

For a side-firing probe, the image is on the same side of the axis as the transducer, so that the axis always lies outside the image region in the image display. For our ultrasound machine, the transducer position is indicated by a horizontal white bar 2 pixels wide located 48 and 49 pixels from the top of the $2 D$ full-screen image, for all FOV settings. Since the axis of rotation is at a fixed physical distance from the transducer, its image location changes with the FOV. Also, since the FOV has a fixed number of pixels, its size in $\mathrm{cm}$ varies directly with pixel size, which can be determined experimentally by imaging an object of known dimensions. Consequently, a plot of $C$ vs $p_{\mathrm{mm}}{ }^{-1}$ should yield a straightline graph with a slope equal to the (negative) physical distance (in $\mathrm{mm}$ ) of the axis from the transducer and an intercept of 48 or 49 pixels. This can be used as a check plot to verify the consistency of the measured values of these quantities. Moreover, the rms deviation of the $C$ values from the best-fit line can be interpreted as the experimental uncertainty in determining the axis location $C$.

\subsection{Results}

\subsubsection{Computer simulation of errors}

\subsubsection{Error in length}

The measured and theoretical values of the relative error $\Delta \ell / \ell$ in the length $\ell$ of the line $L$ shown schematically in Figure 4.5 are shown in Table 4.1 
as a function of the error $\Delta R$ in the axis-to-ROI distance $R_{0}$ used for the $3 D$ image reconstruction, assuming the correct value of $\Theta$ is used; and in Table 4.2 as a function of the error $\Delta \Theta$ in the scanning angle $\Theta$ used for the 3D image reconstruction, assuming the correct value of $R_{0}$ is used. The measured relative error is calculated from the true value of 120 pixels for $e$, and since $L$ is at an angle of $\alpha=90^{\circ}$ to the radial line $\theta=0$, the theoretical relative error is calculated from Eq. (4.8), using $R=152.3$ pixels and $\beta=0.4049$ rad, so that $\beta / \tan \beta=0.945$, as previously described. We remark that the maximum discrepancy of $2.2 \%$ between the measured and theoretical relative errors in Table 4.1 and Table 4.2 could be accounted for by an error of only 3 pixels in measuring $\ell^{\prime}$, which is consistent with an edge width of $n=4$ pixels, as described above and in Appendix $B$.

\subsubsection{Error in area}

The measured and theoretical values of the relative error $\triangle A A$ in the area $A$ of the white square in Figure 4.5 are shown in Table 4.3 as a function of the error $\Delta R$ in axis-to-ROI distance $R_{0}$ used for the 3D image reconstruction, assuming the correct total scanning angle $\Theta$ is used, and in Table 4.4 as a function of error $\Delta \Theta$ in $\Theta$, assuming the correct value of $R_{0}$ is used. The measured relative error is calculated from the true value of $120^{2}$ or 14400 pixels ${ }^{2}$ of $A$, while the theoretical relative error is calculated from Eq. (4.9), using for the mean distance of the square from the axis the value of $R=198.1$ pixels as calculated above. In all cases, the measured and theoretical relative errors are found to agree within $0.6 \%$. 
Table 4.1. Comparison of measured vs predicted relative errors $\Delta \ell / \ell$. in the measured length $\ell^{\prime}=\ell+\Delta \ell$ of line $L$ of Figure 4.5 , whose endpoints have a average radius $R$ of 152.32 pixels. The true length is 120 pixels. Theoretically, $\Delta \ell / \ell=\Delta R / R$.

\begin{tabular}{c|cccc}
\hline \hline$\Delta R$ & $l^{\prime}$ & $\Delta l / l(\%)$ & theory (\%) & difference (\%) \\
\hline-30 & 99 & -17.5 & -19.7 & 2.2 \\
-20 & 106 & -11.7 & -13.1 & 1.4 \\
-10 & 114 & -5.0 & -6.6 & 1.6 \\
0 & 121 & 0.8 & 0.0 & 0.8 \\
10 & 129 & 7.5 & 6.6 & 0.9 \\
20 & 137 & 14.2 & 13.1 & 1.1 \\
30 & 146 & 21.7 & 19.7 & 2.0 \\
\hline \hline
\end{tabular}

Table 4.2. Comparison of measured vs theoretical relative errors $\Delta \ell / \ell$ in the measured length $\ell^{\prime}=\ell+\Delta \ell$ of line $L$ of Figure 4.5. The scanning angle $\Theta$ is $60^{\circ}$. The line $L$ subtends an angle $2 \beta$ at the origin, with $\beta=23.2^{\circ}$, and has a true length of 120 pixels. Theoretically, $\Delta \ell / \ell=\beta / \tan \beta \Delta \Theta / \Theta=0.945 \Delta \Theta / \Theta$.

\begin{tabular}{c|cccc}
\hline \hline$\Delta$ & $l^{\prime}$ & $\Delta l / l(\%)$ & theory (\%) & difference (\%) \\
\hline$-15^{\circ}$ & 92 & -23.3 & -23.6 & 0.3 \\
$-10^{\circ}$ & 102 & -15.0 & -15.8 & 0.8 \\
$-5^{\circ}$ & 112 & -6.7 & -7.8 & 1.1 \\
$0^{\circ}$ & 121 & 0.8 & 0.0 & 0.8 \\
$5^{\circ}$ & 130 & 8.3 & 7.8 & 0.5 \\
$10^{\circ}$ & 140 & 16.7 & 15.8 & 0.9 \\
$15^{\circ}$ & 150 & 25.0 & 23.6 & 1.4 \\
\hline \hline
\end{tabular}


Table 4.3. Comparison of measured vs predicted relative errors $\triangle A / A$ in the measured area $A^{\prime}=A+\Delta A$ of the square of Figure 4.5, whose average radius $R$ is 198.1 pixels. The true area $A$ is 14000 pixels. Theoretically, $\triangle A A=\Delta R / R$.

\begin{tabular}{c|cccc}
\hline$\Delta R$ & $A^{\prime}$ & $\Delta H / A(\%)$ & theory (\%) & difference (\%) \\
\hline-30 & 12238 & -15.0 & -15.1 & 0.1 \\
-20 & 12970 & -9.9 & -10.1 & 0.2 \\
-10 & 13668 & -5.1 & -5.0 & -0.1 \\
0 & 14480 & 0.6 & 0.0 & 0.6 \\
10 & 15144 & 5.2 & 5.0 & 0.2 \\
20 & 15862 & 10.2 & 10.1 & 0.1 \\
30 & 16596 & 15.3 & 15.1 & 0.2 \\
\hline \hline
\end{tabular}

Table 4.4. Comparison of measured vs theoretical relative errors $\triangle A A$ in the measured area $A^{\prime}=A+\Delta A$ of the square in Figure 4.5. The true area $A$ is 14400 pixels, and the scanning angle $\Theta$ is $60^{\circ}$. Theoretically, $\Delta A / A=\Delta \odot / \Theta$.

\begin{tabular}{c|cccc}
\hline \hline$\Delta \Theta$ & $A^{\prime}$ & $\Delta-A / A)$ & theory (\%) & difference (\%) \\
\hline$-15^{\circ}$ & 10823 & -24.8 & -25.0 & 0.2 \\
$-10^{\circ}$ & 12034 & -16.4 & -16.7 & 0.3 \\
$-5^{\circ}$ & 13155 & -8.6 & -8.3 & -0.3 \\
$0^{\circ}$ & 14480 & 0.6 & 0.0 & 0.6 \\
$5^{\circ}$ & 15615 & 8.4 & 8.3 & 0.1 \\
$10^{\circ}$ & 16773 & 16.5 & 16.7 & -0.2 \\
$15^{\circ}$ & 18045 & 25.3 & 25.0 & 0.3 \\
\hline \hline
\end{tabular}




\subsubsection{Experimental measurement of errors}

In Figure 4.7, an undistorted $x-y$ cross-section of the 3D wire phantom image is shown, and in Figure 4.8 and Figure 4.9, the middle row from similar, but distorted, $x-y$ cross-sections are shown. In Figure 4.8, these images were reconstructed using values for the axis-to-ROI distance $R_{0}^{\prime}$ of $50,60,70,82,90$, 100 , and 110 pixels in the top to bottom panels, respectively, with the correct value of $60.14^{\circ}$ being used for the total scanning angle $\Theta$; while, in Figure 4.9, they were reconstructed using values for $\Theta^{\prime}$ of $45^{\circ}, 50^{\circ}, 55^{\circ}, 60.14^{\circ}, 65^{\circ}, 70^{\circ}$, and $75^{\circ}$ in the top to bottom panels, respectively, with the correct value of 82.0 pixels being used for $R_{0}$.

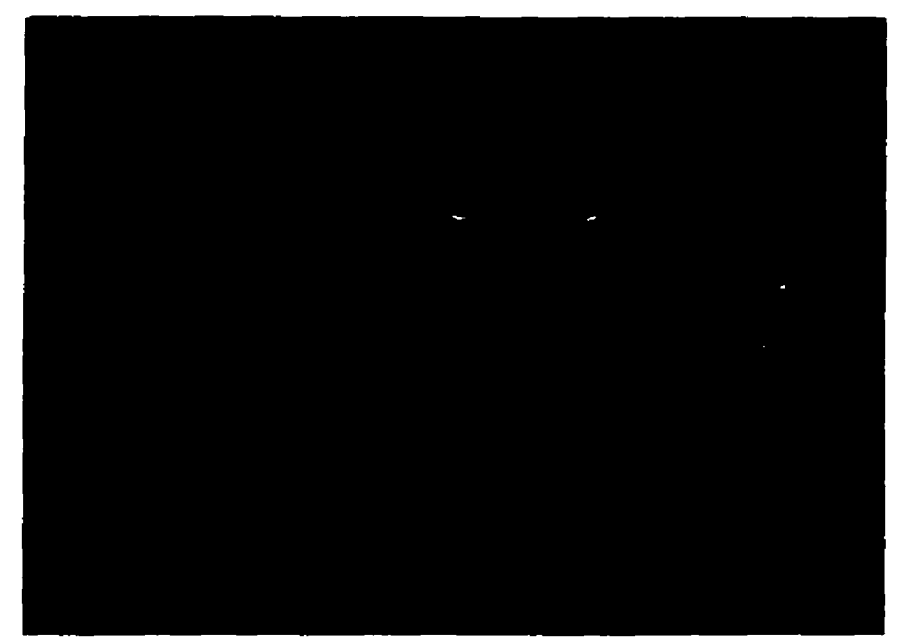

Figure 4.7. An $x-y$ cross-section of the $3 D$ ultrasound image of the wire phantom, containing $254 \times 184$ square pixels of size $0.257 \mathrm{~mm} /$ pixel in each direction. Here, although the wire images are stretched in the direction of rotation, due (overwhelmingly) to the finite thickness of the $2 \mathrm{D}$ image slices and (slightly) to interpolation effects, so that the image is aberrated, their centroids are correctly located, so that it is undistorted. This image was reconstructed using (correct) parameter values of $\Theta=60.14^{\circ}$ for the total scanning angle and $R_{0}=82.0$ pixels for the inner radius of the fan of $2 \mathrm{D}$ image ROIs, each parallel to the $z$ axis, which are reconstructed into the $3 \mathrm{D}$ image. This axis (the probe rotation axis) intersects the image plane outside the frame, at a point 163 pixels above the image center. 


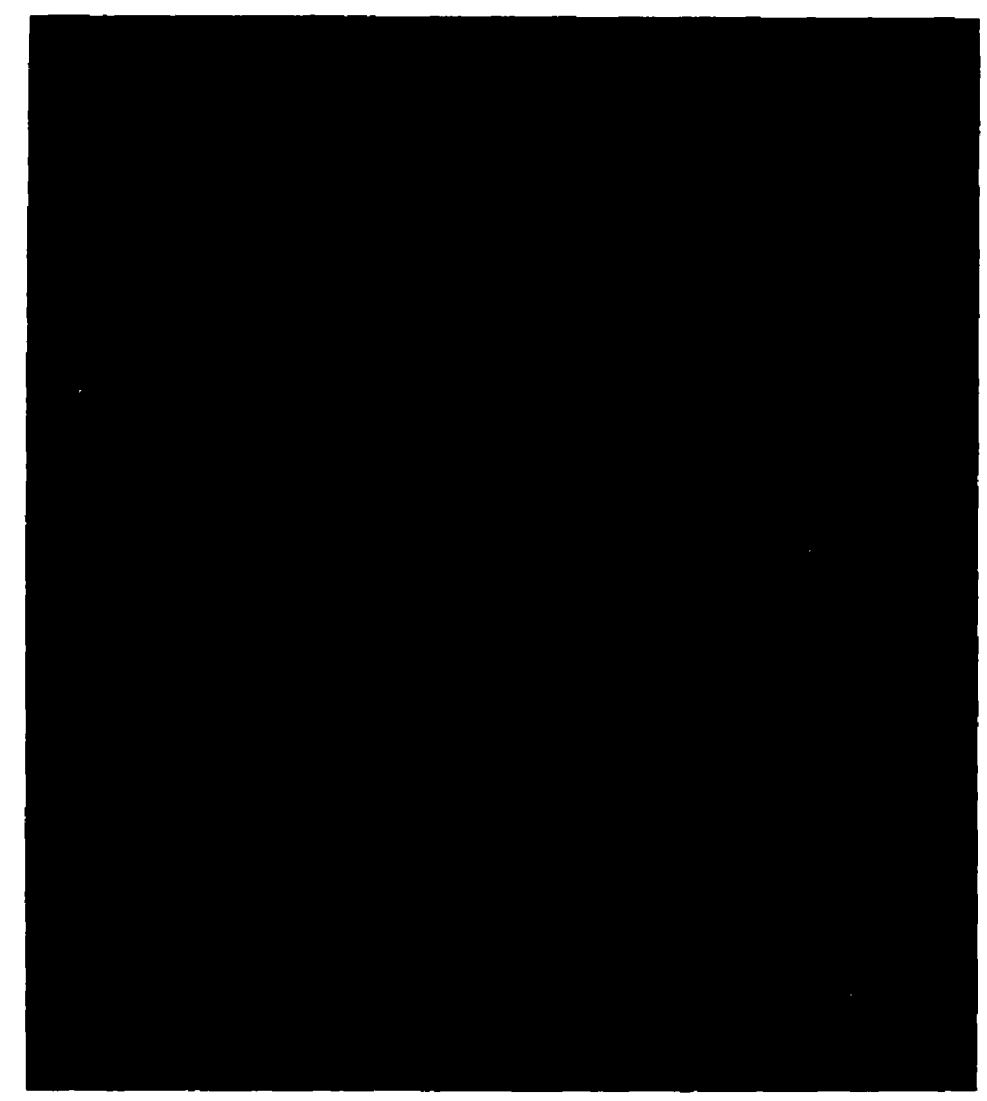

Figure 4.8. Central portions of $x-y$ cross-sections of $3 D$ ultrasound images of the wire phantom, similar to Figure 4.7, but reconstructed using a range of parameter values $R_{0}^{\prime}$ for the inner radius of the fan of 2D image ROls, together with the (correct) parameter value of $\Theta=60.14^{\circ}$ for the total scanning angle. From top to bottom, $R_{0}{ }^{\prime}=50,60,70,82,90,100$, and 110 pixels. The correct value is $R_{0}=82.0$ pixels. 


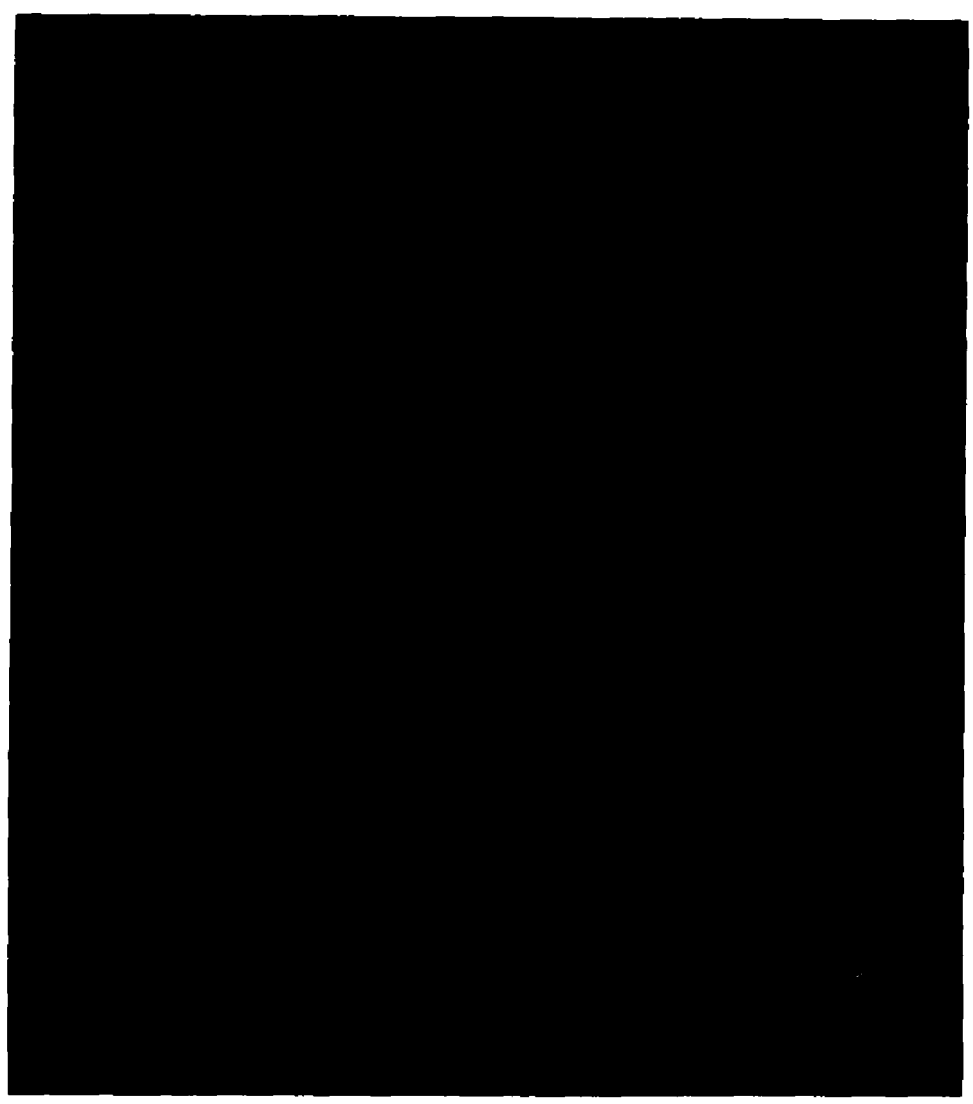

Figure 4.9. Central portions of $x-y$ cross-sections of 3D ultrasound images of the wire phantom, similar to Figure 4.7, but reconstructed using a range of parameter values $\odot$ for the total scanning angie, together with the (correct) parameter value of $R_{0}=82.0$ pixels for the inner radius of the fan of $2 \mathrm{D}$ image ROls. From top to bottom, $\Theta^{\prime}=45^{\circ}, 50^{\circ}, 55^{\circ}, 60.14^{\circ}, 65^{\circ}, 70^{\circ}$, and $75^{\circ}$. The correct value is $\Theta=60.14^{\circ}$. 
Since the distance $R$ from the axis to the middle row of wires is about 170 pixels, the relative error of $\Delta R / R$ is about $-19 \%,-13 \%,-7 \%, 0 \%, 5 \%, 11 \%$, and $16 \%$, respectively, in the top to bottom panels of Figure 4.8; while the relative error $\Delta \Theta / \Theta$ is about $-25 \%,-17 \%,-9 \%, 0 \%, 8 \%, 16 \%$, and $25 \%$, respectively, in the top to bottom panels of Figure 4.9. From these figures, it can be seen that image distortions in line shape and length increase in proportion to $\Delta R / R$ or $\Delta \Theta / \Theta$, since the wires describe a straight line of the correct length in the middle (undistorted) panel. Moreover, the magnitude of the distortions shown in Figure 4.8 for an error of $\Delta R \approx \pm 30$ pixels (first and last panels) is similar to that shown in Figure 4.9 for an error of $\Delta \Theta \approx \pm 10^{\circ}$ (second and second-last panels), so that, here, an error of $\Delta R \approx \pm 3$ pixels is roughly equivalent to an error of $\Delta \Theta \approx \pm 1^{\circ}$ in the reconstruction parameters $R_{0}$ and $\Theta$, respectively.

\subsubsection{Distortion in the image of a straight line}

In Figure 4.10, the maximum relative distortion $h / \ell$ in the image of the line described by three collinear wires in the phantom (the middle three wires of the middle row in Figure 4.7) is plotted as a function of $\Delta R$ and $\Delta \Theta$, for $|\Delta R| \leq 10$ pixels, with $\Delta \Theta=0$, and $|\Delta \Theta| \leq 5^{\circ}$, with $\Delta R=0$. Experimental values are shown by $x$ 's for the former series and o's for the latter, with error bars determined via Eq. (4.15), where $\sigma_{y}=0.12 \mathrm{~mm}$ is the experimental standard deviation in the $y$-coordinate of the image centroid (Tong et al. 1996), and $\ell=20 \mathrm{~mm}$ is the separation of the end wires in the phantom. The corresponding theoretical values, calculated via Eq. (4.13), and plotted as solid and dashed lines for $\Delta R \neq 0$ and $\Delta \Theta \neq 0$, respectively, always lie within these error bars. 


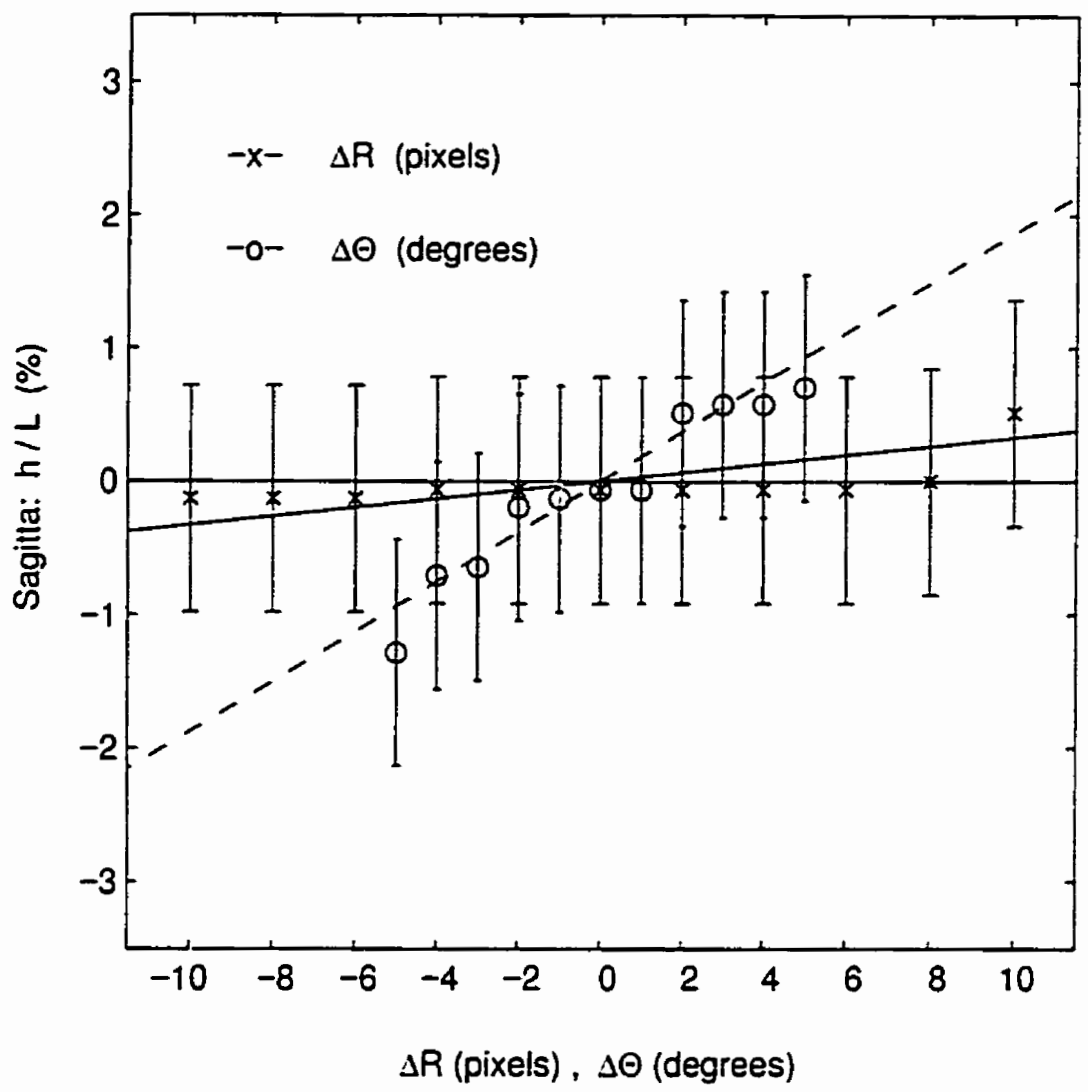

Figure 4.10. Graph of the maximum relative perpendicular distortion $h / \ell$ in the reconstructed image of a straight line perpendicular to and bisected by the radial line $\theta=0$, plotted as a function of the error $\Delta R=R_{0}{ }^{\prime}-R_{0}$ in the value of the inner radius of the fan of 2D image ROls used for the $3 D$ image reconstruction, with the correct parameter value $\Theta=60.14^{\circ}$; and as a function of the error $\Delta \Theta=\Theta^{\prime}-\Theta$ in the total scanning angle used for the 3D image reconstruction, with the correct parameter value $R_{0}=82.0$ pixels. Theoretical values of $h / \ell$, indicated by solid and dashed lines, respectively, were calculated via Eq. (4.13), using values of $R=172.8$ pixels for the (average) radius of the line endpoints, and $\beta=0.2253$ rad for the semi-angle subtended by the line at the axis. The error bars were calculated via Eq. (4.15), using a measurement uncertainty of $\sigma_{y}=0.12 \mathrm{~mm}$ (Tong et al. 1996), and an undistorted line length of $\ell=20 \mathrm{~mm}$. 


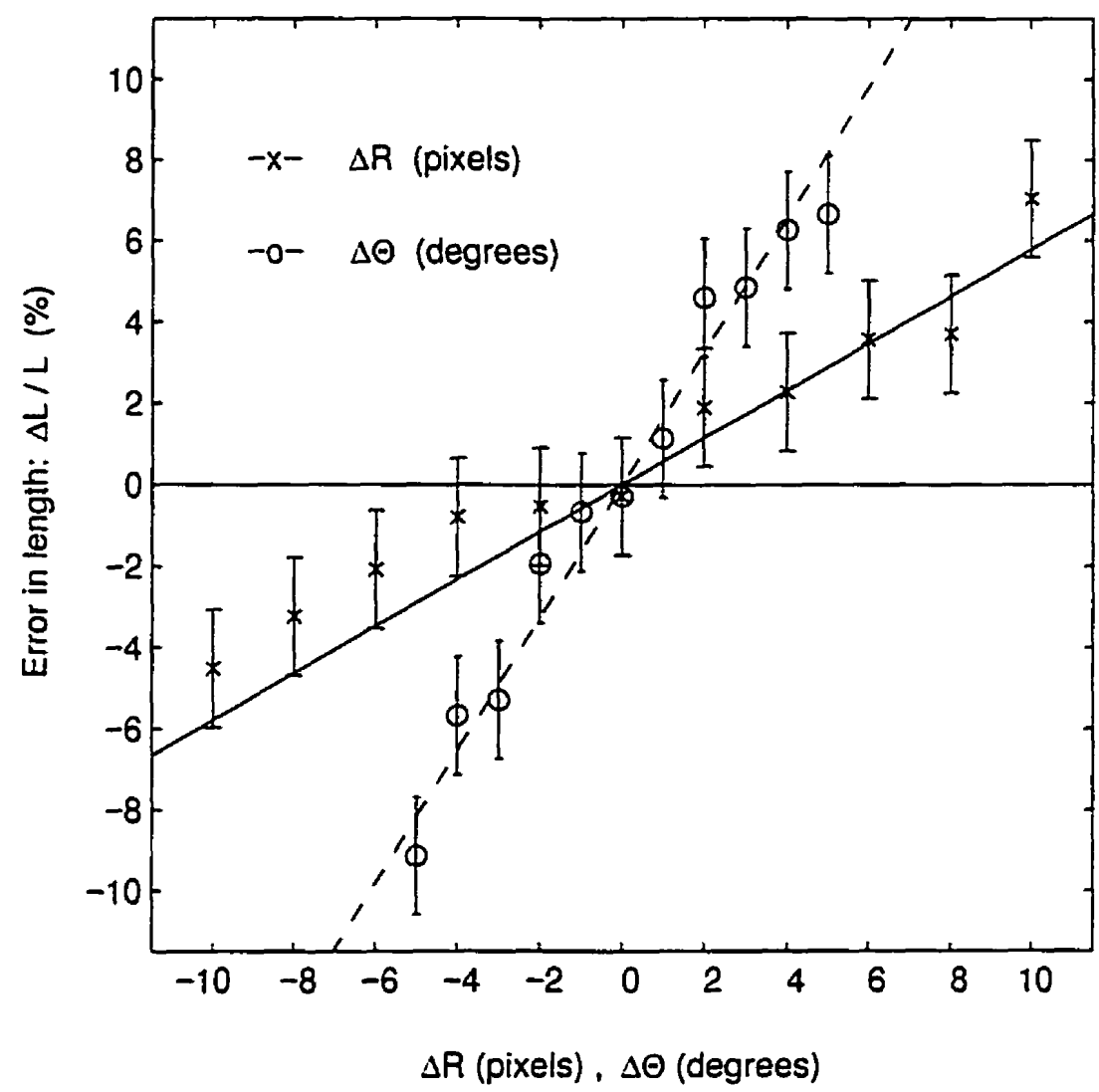

Figure 4.11. Graph of the relative distortion $\Delta \ell / \ell$ in the length of the reconstructed image of a straight line perpendicular to and bisected by the radial line $\theta=0$, plotted as a function of the error $\Delta R=R_{0}{ }^{\prime}-R_{0}$ in the value of the inner radius of the fan of $2 D$ image ROls used for the $3 D$ image reconstruction, with the correct parameter value $\Theta=60.14^{\circ}$; and as a function of the error $\Delta \Theta=\Theta^{\prime}-\Theta$ in the total scanning angle used for the 3D image reconstruction, with the correct parameter value $R_{0}=82.0$ pixels. Theoretical values of $\Delta \ell / \ell$, indicated by solid and dashed lines, respectively, were calculated via Eq. (4.8), using values of $R=172.8$ pixels for the average radius of the line endpoints, and $\beta=0.2253 \mathrm{rad}$ for the semi-angle subtended by the line at the axis. The error bars were calculated via Eq. (4.16), using a measurement uncertainty of $\sigma_{\mathrm{x}}=0.21 \mathrm{~mm}$ (Tong et al. 1996), and an undistorted line length of $\ell=20 \mathrm{~mm}$. 


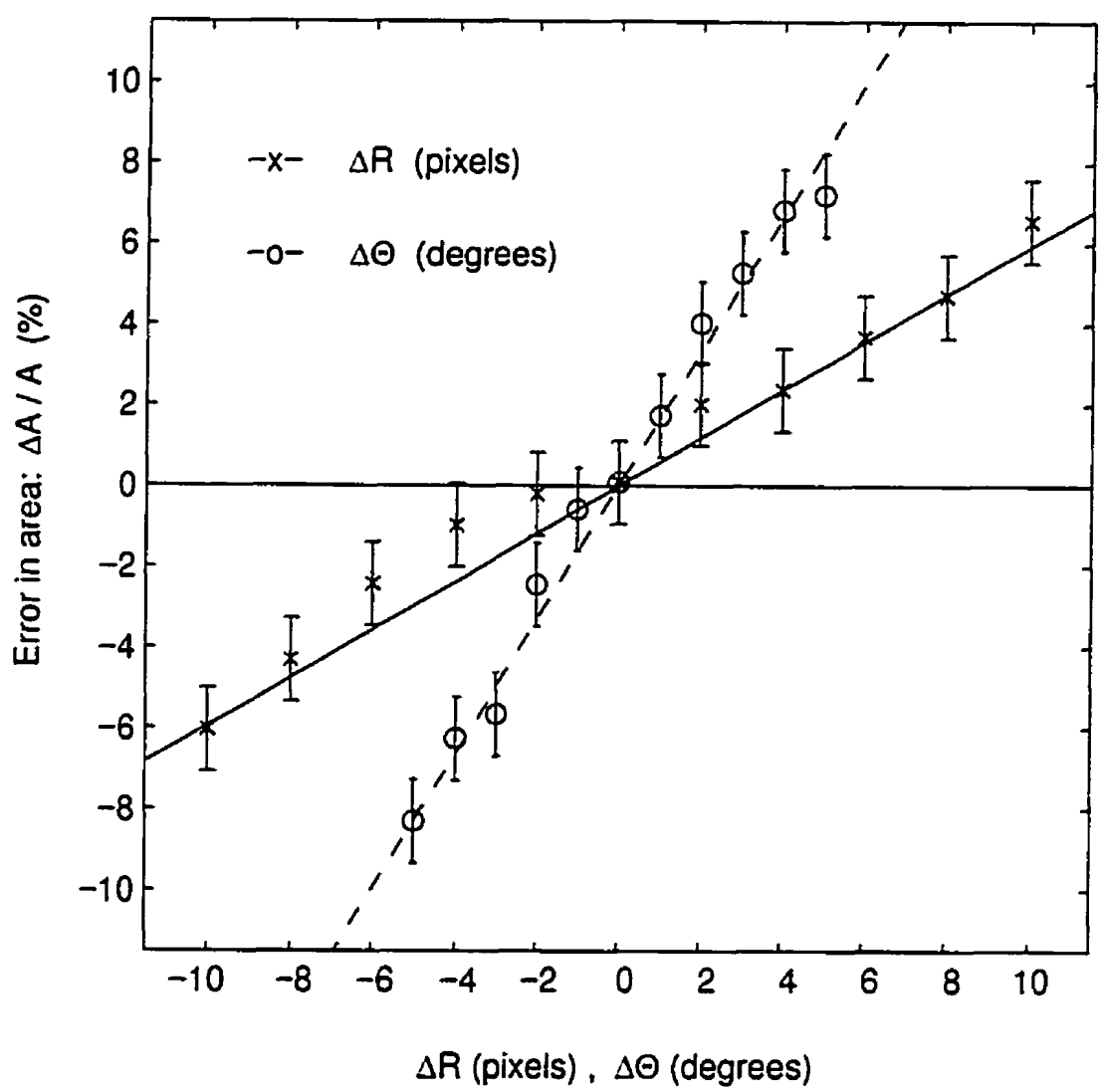

Figure 4.12. Graph of the relative distortion $\Delta A / A=\left(A^{\prime}-A\right) / A$ in the area of the parallelogram containing the central nine centroids of Figure 4.7 is plotted as a function of the error $\Delta R=R_{0}{ }^{\prime}-R_{0}$ in the value of the inner radius of the fan of $2 D$ image ROls used for the $3 D$ image reconstruction, with the correct parameter value $\Theta=60.14^{\circ}$; and as a function of the error $\Delta \Theta=\Theta^{\prime}-\Theta$ in the total scanning angle used for the 3D image reconstruction, with the correct parameter value $R_{0}=82.0$ pixels. The measured area $A^{\prime}$ was calculated via Eq. (4.17), while the undistorted area $A$ was calculated as $77.8^{2}$ pixels ${ }^{2}$ from the image scale of $0.257 \mathrm{~mm} /$ pixel. Theoretical values of $\Delta A / A$, indicated by solid and dashed lines, respectively, were calculated via Eq. (4.9), using the radius 168.3 pixels of the central centroid as the average distance $R$ of $A$ from the axis. The error bars were calculated via Eq. (4.18), using measurement uncertainties of $\sigma_{x}=0.21 \mathrm{~mm}$ and $\sigma_{y}=0.12 \mathrm{~mm}$ (Tong et al. 1996), and an undistorted area of $A=20^{2} \mathrm{~mm}^{2}$. 


\subsubsection{Error in length}

In Figure 4.11, the relative error $\Delta \ell / \ell$ in the length of the image of the same line is plotted as a function of $\Delta R$ and $\Delta \Theta$. Here, the error bars are determined by Eq. (4.16), where $\sigma_{x}=0.21 \mathrm{~mm}$ is the experimental standard deviation in the $x$-coordinate of the image centroid (Tong et al. 1996) and again $\ell=20 \mathrm{~mm}$; while the corresponding theoretical values were calculated via Eq. (4.8), with $R=172.8$ pixels, $\beta=0.2253 \mathrm{rad}$, and $\beta / \tan \beta=0.983$, as described above. Again, the theoretical values always lie within the experimental error bars.

\subsubsection{Error in area}

In Figure 4.12, the relative error $\triangle A / A$ in the area of the 8-sided polygon formed by the images of the middle three wires of the middle three rows of Figure 4.7 is also plotted as a function of $\Delta R$ and $\Delta \Theta$. Here, the area $A^{\prime}$ of the distorted polygon was calculated via Eq. (4.17) and the error bars determined by Eq. (4.18), where again $\sigma_{x}=0.21 \mathrm{~mm}$ and $\sigma_{y}=0.12 \mathrm{~mm}$, and $A=400 \mathrm{~mm}^{2}$ is the area of the corresponding phantom cross-section, while the corresponding theoretical values were calculated via Eq. (4.9), with $R=168.3$ pixels, the distance of the center wire from the axis. Again, the theoretical values always lie within the experimental error bars.

\subsubsection{Determination of axis location}

For each available FOV setting, the axis location $C$, measured in pixels from the top edge of the full-screen $2 D$ image, and the corresponding pixel size $p_{\mathrm{mm}}$, were determined via the experimental procedure described in the previous section, which was also used to determine that the latex plane thickness $d_{\mathrm{mm}}$ is $0.16 \pm 0.01 \mathrm{~mm}$ for our phantom. These results are tabulated in Table 4.5, and the check plot of axis location $C$ vs image resolution $p_{\mathrm{mm}}{ }^{-1}$ is shown in Figure 4.13, with the least-squares linear regression through the data points shown as a solid line. The slope of this line, $-5.82 \mathrm{~mm}$, corresponds to the 
(negative of the) physical distance from the axis to the transducer crystal face, while its intercept, 49.6 pixels, corresponds to the image position assigned to the crystal face by the ultrasound machine software, as indicated by the white horizontal bar which appears at 48 and 49 pixels from the top edge of each fullscreen 2D image. The correlation coefficient of 0.996 shows that the experimentally determined values of axis location and pixel size are remarkably consistent. Also, the rms deviation of the axis location values from the best-fit line is 0.3 pixels, which is thus the experimental standard deviation in the axis location for each FOV, and hence the size of the error bars shown in Figure 4.13.

Table 4.5. Experimentally measured location of the probe rotation axis (relative to the top of the 2D full-screen image) for different pixel sizes (varied by adjusting the field-of-view (FOV) setting).

\begin{tabular}{ccc}
\hline $\begin{array}{c}\text { Nominal FOV } \\
(\mathrm{cm})\end{array}$ & $\begin{array}{c}\text { Pixel size } \\
(\mathrm{mm})\end{array}$ & $\begin{array}{c}\text { Axis location } \\
\text { (pixels) }\end{array}$ \\
\hline 4.5 & 0.140 & 8.0 \\
5.5 & 0.160 & 12.9 \\
6.5 & 0.187 & 18.7 \\
7.0 & 0.210 & 22.0 \\
7.5 & 0.224 & 24.2 \\
9.0 & 0.257 & 27.0 \\
10.0 & 0.294 & 30.1 \\
11.0 & 0.327 & 31.8 \\
13.0 & 0.374 & 33.9 \\
14.0 & 0.411 & 35.2 \\
\hline \hline
\end{tabular}




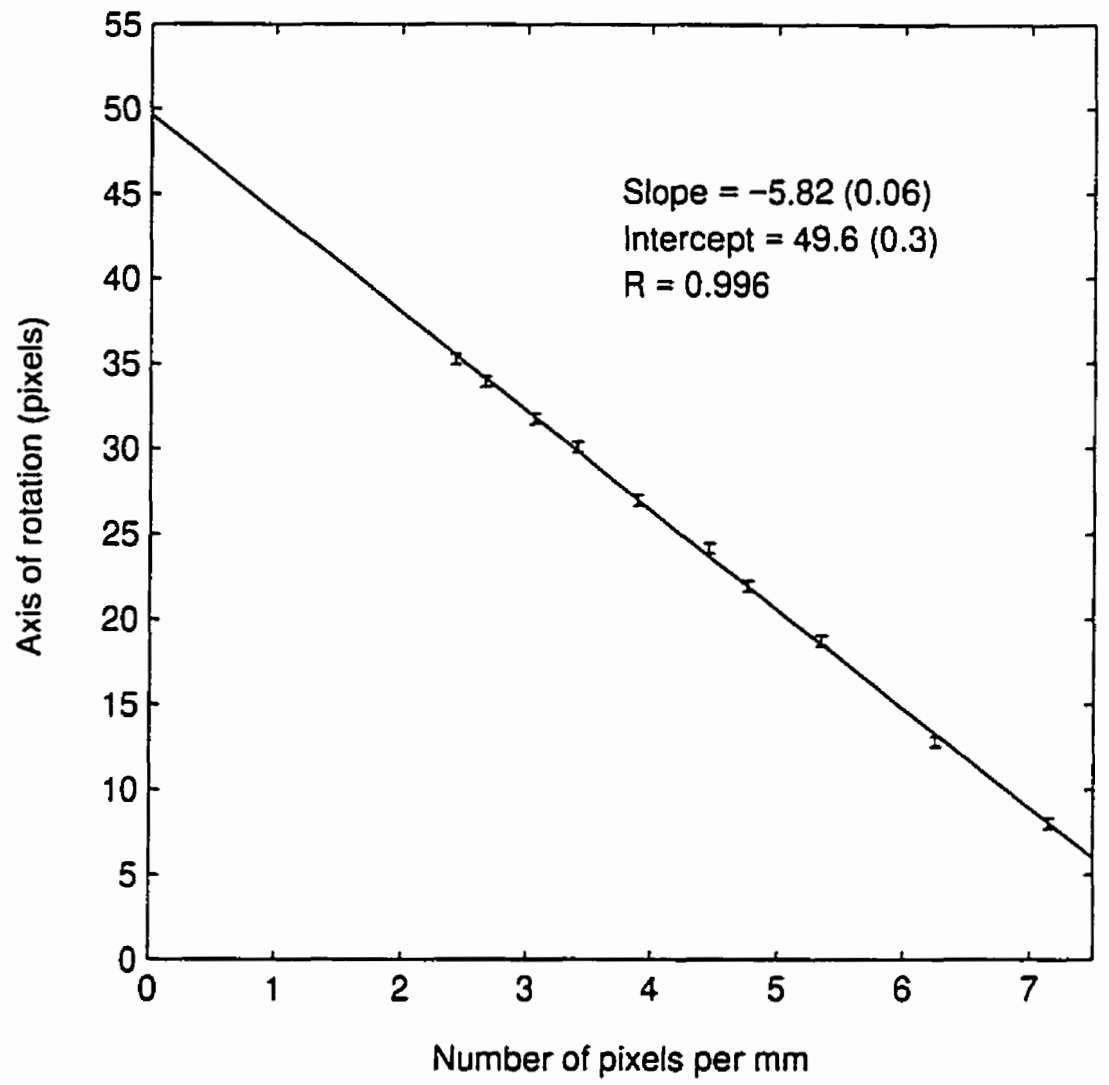

Figure 4.13. Plot of axis location vs reciprocal pixel size. For each field of view (FOV) setting on the ultrasound machine, the pixel size $p_{\text {mrm }}$ was calculated via Eq. (4.20), using $D_{\mathrm{mm}}=30.00 \mathrm{~mm}, d_{\mathrm{mm}}=0.16 \mathrm{~mm}$, and the measured locations of latex planes $A_{1}, A_{2}, B_{1}$, and $B_{2}$ of the calibration phantom described in Figure 4.6. The corresponding axis location $C$, measured in pixels from the top of each $2 \mathrm{D}$ full-screen image, was then found via Eq. (4.21). The least-squares regression line has a slope of $-5.82 \pm 0.06 \mathrm{~mm}$, an intercept of $49.6 \pm 0.3$ pixels, and a correlation coefficient of 0.996 . The rms deviation of the measured axis locations from this line is 0.3 pixels, which is the size of the error bars shown.

\subsection{Discussion and Conclusions}

For a 3D ultrasound imaging system using a side-firing probe, we have shown that there are only two potentially significant sources of error among the parameters used to define the $3 D$ image reconstruction algorithm: an error $\Delta R$ in 
the parameter $R_{0}$, the distance of the probe rotation axis from the image ROI; or, an error $\Delta \Theta$ in the parameter $\Theta$, the total angle through which the probe is rotated during a scan. Although differences in the speed of sound between different types of soft tissues and effect of refraction could be potential sources of error, we expect that the effect is small, since the speed of sound varies within $5 \%$ for different tissue types and the distance from the ultrasound transducer to the prostate gland is only a few $\mathrm{cm}$.

As a result of these errors, $\Delta R$ and $\Delta \Theta$, four types of image distortions appear in the reconstructed 3D image: (i) the relative distortion in the shape of the image of a straight line, as measured by the ratio $h / \ell$ of the perpendicular displacement $h$ (the height of the triangle formed by an intermediate point and the two endpoints of the line image) to the undistorted length $\ell$ of the line; (ii) the relative distortion $\Delta \ell / \ell$ in $\ell$; (iii) the relative distortion $\Delta A / A$ in the area $A$ of an arbitrary object cross-section, and (iv) the relative distortion $\triangle V I V$ in the volume $V$ of an arbitrary object, which is identical to $\Delta A / A$, since all distances in the axial direction are known precisely.

Assuming that the angular spacing between successive $2 \mathrm{D}$ image acquisitions remains uniform as the probe is rotated, so that the relative error $\Delta \theta / \theta$ in any angle $\theta$ is also uniform and equal to $\Delta \Theta / \Theta$, we have theoretically derived closed-form analytic expressions for each of these image distortions. In every case, the relative error is well-described by the bilinear form $P \Delta R / R+Q \Delta \Theta / \Theta$, where $|P|<1$ and $|Q|<1$ in cases (i) and (ii), $P=Q=1$ in cases (iii) and (iv), and $R$ is the average distance of the object from the axis, which is analytically defined for an arbitrary straight line, cross-sectional area, or volume. For a line, $R$ is the average distance of the endpoints from the axis; while, for an area or volume, we have demonstrated numerically that $R$ is closely approximated by the distance of the object's geometric center from the axis.

In order to verify numerically and experimentally the accuracy of our theoretical formulas, these distortions were measured in simulated images of a 
white cube and in real images of a wire phantom, and the results compared to the theoretical values. In all cases, the measured and theoretical values agreed within the measurement uncertainty, confirming the accuracy of the latter.

It is therefore clear that it is possible to virtually eliminate all $3 D$ image distortions by using a suitable calibration procedure to determine accurate values for the parameters $R_{0}$ and $\Theta$, so that $|\Delta R / R| \ll 1$ and $|\Delta \Theta / \Theta| \ll 1$. Here, we have described how a simple calibration phantom can be used to determine the axis location $C$ (in pixels) from the top edge of the full-screen $2 D$ image with sub-pixel accuracy, together with the corresponding pixel size $p_{\mathrm{mm}}$ (in $\mathrm{mm}$ ), as functions of the FOV setting. A plot of $C$ vs $p_{\mathrm{mm}}{ }^{-1}$ then yields a straight-line graph whose slope is the negative of the physical distance (in $\mathrm{mm}$ ) of the transducer crystal face from the axis; whose intercept (in pixels) is the transducer location in each full-screen 2D image (indicated by a white bar for our machine); and whose rms deviation from the least-squares regression line is the experimental uncertainty in $C$. Given $C$, we can then calculate $R_{0}=Y_{0}-C$, where, for any chosen ROI, $Y_{0}$ is the location (in pixels) of the top of the ROI in each full-screen $2 D$ image.

Once $R_{0}$ and $\rho_{\mathrm{mm}}$ are known, a wire phantom can be used to calibrate $\Theta$, as follows: Orienting the phantom wires parallel to the axis, scan the phantom through an estimated total scanning angle $\Theta^{\prime}$, reconstruct the 3D image, and measure the image centroids $\left(R_{1}, \theta^{\prime}{ }_{1}\right)$ and $\left(R_{2}, \theta^{\prime}{ }_{2}\right)$ of two wire images in order of increasing $\theta$, so that their angular separation $\theta^{\prime}{ }_{2}-\theta^{\prime}{ }_{1}$ is positive. Now, let $\ell \mathrm{mm}$ be the corresponding physical separation of the two wires in the phantom. Then, in pixels, the correct image separation is simply $\ell=\ell_{\mathrm{mm}} / p_{\mathrm{mm}}$, and, since the radii $R_{1}$ and $R_{2}$ are already correct, the correct angular separation $\theta_{2}-\theta_{1}$ of the two wire images is the physical angle subtended by the two wires at the axis, which is

$$
\theta_{2}-\theta_{1}=\arccos \left(\frac{R_{1}^{2}+R_{2}^{2}-\ell^{2}}{2 R_{1} R_{2}}\right)
$$


by the law of cosines. The correct value $\Theta$ of the total scanning angle is then simply

$$
\Theta=\left(\frac{\theta_{2}-\theta_{1}}{\theta_{2}^{\prime}-\theta_{1}^{\prime}}\right) \Theta^{\prime}
$$

Thus, for the greatest accuracy in calculating $\Theta$, it is best to make the angular separation $\theta_{2}-\theta_{1}$ and radii $R_{1}$ and $R_{2}$ as large as feasible.

In conclusion: (i) we have investigated the sources and character of image artifacts for 3D ultrasound imaging systems which use a side-firing probe to obtain a series of $2 \mathrm{D}$ images by rotationally scanning a volume of interest; (ii) we have identified the two scanning parameters, the inner radius $R_{0}$ of each $2 \mathrm{DOI}$ and the total scanning angle $\Theta$, whose values must be correctly ascertained in order to avoid distortions in the reconstructed 30 image; (iii) we have identified four types of distortion (shape, length, area, and volume), mathematically characterized them via simple theoretical formulas, and numerically and experimentally verified the accuracy of these formulas; finally, (iv) we have described practical calibration procedures for determining accurate values for $R_{0}$ and $\Theta$, and thereby reducing all four types of distortion to negligible proportions. 


\subsection{Appendices}

\section{A.1 Distortion in the image of a straight line}

Consider a line $L$ in an $x-y$ plane, and let the intersection of the $z$, or probe, axis with this plane be the origin $O$ of polar coordinates $(r, \theta)$ and Cartesian coordinates $(x, y)$ in this plane. Further, let $P_{1}$ and $P_{2}$ denote the endpoints of $L$, chosen so that $\theta$ increases in going from $P_{1}$ to $P_{2}$, and define $R_{1}=\left|O P_{1}\right|, R_{2}=\left|O P_{2}\right|, R=\left(R_{2}+R_{1}\right) / 2$, and $S=\left(R_{2}-R_{1}\right) / 2$, (see Figure 2.2). Also, denote the angle $\angle P_{1} O P_{2}$ by $2 \beta$, and define its bisector as the polar axis $\theta=0$, so that $P_{1}$ and $P_{2}$ have polar coordinates $\left(R_{1},-\beta\right)$ and $\left(R_{2}, \beta\right)$, respectively, and denote the angle between $L$ and the polar axis as $\alpha$, where $0 \leq \alpha<\pi$. The equation of $L$ can then be written in polar coordinates as

$$
r \sin (\alpha-\theta)=D
$$

where $|\theta| \leq \beta<\min (\alpha, \pi-\alpha)$ and $D$ is the perpendicular distance from $O$ to $L$. Now, since $P_{1}$ and $P_{2}$ lie on $L$, we have that

$$
\begin{aligned}
& R_{1} \sin (\alpha+\beta)=D \\
& R_{2} \sin (\alpha-\beta)=D,
\end{aligned}
$$

and hence

$$
\begin{aligned}
& R=\frac{R_{2}+R_{1}}{2}=\frac{D \sin \alpha \cos \beta}{\sin (\alpha-\beta) \sin (\alpha+\beta)}, \\
& S=\frac{R_{2}-R_{1}}{2}=\frac{D \cos \alpha \sin \beta}{\sin (\alpha-\beta) \sin (\alpha+\beta)} . \\
& \tan \alpha=\frac{R}{S} \tan \beta,
\end{aligned}
$$

Moreover, since $(x, y)=(r \cos \theta, r \sin \theta)$, we have from the definition of $\alpha$ that

$$
\begin{aligned}
& x_{2}-x_{1}=\left(R_{2}-R_{1}\right) \cos \beta=2 S \cos \beta=\ell \cos \alpha, \\
& y_{2}-y_{1}=\left(R_{2}+R_{1}\right) \sin \beta=2 R \sin \beta=\ell \sin \alpha,
\end{aligned}
$$


where $e=\left|P_{1} P_{2}\right|=\sqrt{\left(x_{2}-x_{1}\right)^{2}+\left(y_{2}-y_{1}\right)^{2}}$ is the length of $L$, so that

$$
D=\frac{\ell \cdot \sin (\alpha-\beta) \sin (\alpha+\beta)}{2 \sin \beta \cos \beta}=\frac{\ell \cdot\left(\sin ^{2} \alpha-\sin ^{2} \beta\right)}{\sin 2 \beta} \text {. }
$$

Now, if the inner radius $R_{0}$ of the ROI in each $2 D$ image is incorrectly estimated as $R_{0}^{\prime}=R_{0}+\Delta R$, then every point in the plane of $L$ is incorrectly remapped away from $(\Delta R>0)$ or towards $(\Delta R<0)$ the origin $r=0$ by the same radial distance $|\Delta R|$. Thus, in particular, we may consider $\Delta R=R^{\prime}-R$, while $\Delta S=S^{\prime}-S=0$.

Similarly, if the total scanning angle $\Theta$ is incorrectly estimated as $\Theta^{\prime}=\Theta+\Delta \Theta$, then, assuming that, to good approximation, the angular interval between successive $2 D$ image acquisitions remains uniform throughout the scan, every point in the plane of $L$ is incorrectly remapped away from $(\Delta \Theta>0)$ or towards $(\Delta \Theta<0)$ the polar axis $\theta=0$ by an angle $\Delta \theta$ proportional to its angular coordinate $\theta$. Thus, in particular, $\Delta \beta=\beta^{\prime}-\beta=\beta \Delta \Theta / \Theta$, so that the relative error $\Delta \beta / \beta=\Delta \theta / \theta=\Delta \Theta / \Theta$ is constant.

As a consequence, the image $L^{\prime}$ of $L$ will change both in shape and length. We consider the former effect here, and the latter in the next section. The amount of distortion of a straight line is best described by the perpendicular height $h$ of the triangle $P_{1}^{\prime} P^{\prime} P_{2}^{\prime}$ consisting of a point $P^{\prime}$ on $L^{\prime}$ and its two endpoints $P_{1}{ }^{\prime}$ and $P_{2}{ }^{\prime}$. Thus, as $\theta$ increases from $-\beta$ to $\beta, P$ traverses $L$ from $P_{1}$ to $P_{2}$ and $P^{\prime}$ traverses $L^{\prime}$ from $P_{1}{ }^{\prime}$ to $P_{2}{ }^{\prime}$. Hence, $h=0$ when $\theta= \pm \S$ or when $P^{\prime}$ coincides with $P_{1}{ }^{\prime}$ and $P_{2}{ }^{\prime}$, and $h>0$ if the apex of the triangle points away from the axis, in the positive $r$ direction. Also, by definition, the sagitta $s$ of $L^{\prime}$ is the maximum magnitude of $h$.

To derive a suitable mathematical expression for $h$, we first note that the polar and Cartesian coordinates of $P$ are related to those of $P$ via the equations:

$$
r^{\prime}=r+\Delta R=\frac{D}{\sin (\alpha-\theta)}+\Delta R
$$




$$
\begin{aligned}
& \theta^{\prime}=\theta+\Delta \theta=\theta\left[1+\frac{\Delta \Theta}{\Theta}\right], \\
& x^{\prime}=r^{\prime} \cos \theta^{\prime}=\frac{D \cos \theta}{\sin (\alpha-\theta)}\left[1+\frac{R \sin (\alpha-\theta)}{D} \frac{\Delta R}{R}\right]\left[1-\theta \tan \theta \frac{\Delta \Theta}{\Theta}\right], \\
& y^{\prime}=r^{\prime} \sin \theta^{\prime}=\frac{D \sin \theta}{\sin (\alpha-\theta)}\left[1+\frac{R \sin (\alpha-\theta)}{D} \frac{\Delta R}{R}\right]\left[1+\theta \cot \theta \frac{\Delta \Theta}{\Theta}\right],
\end{aligned}
$$

where the last two equations are correct to first order in $\Delta \Theta / \Theta$, and similarly for the endpoints $P_{1}^{\prime}(\theta=-\beta)$ and $P_{2}(\theta=\beta)$. We then note that the area $A^{\prime}$ of triangle $P_{1}^{\prime} P^{\prime} P_{2}^{\prime}$ is given by

$$
A^{\prime}=\frac{1}{2}\left|\begin{array}{lll}
x_{2}^{\prime} & y_{2}^{\prime} & 1 \\
x_{1}^{\prime} & y_{1}^{\prime} & 1 \\
x^{\prime} & y^{\prime} & 1
\end{array}\right|=\frac{1}{2}\left[\left(y_{2}^{\prime}-y_{1}^{\prime}\right) x^{\prime}-\left(x_{2}^{\prime}-x_{1}^{\prime}\right) y^{\prime}+x_{2}^{\prime} y_{1}^{\prime}-x_{1}^{\prime} y_{2}^{\prime}\right],
$$

and also by

$$
A^{\prime}=\frac{1}{2} h \ell^{\prime} \approx \frac{1}{2} h \ell^{\prime},
$$

where $\ell^{\prime}$ is the length $\left|P_{1}^{\prime} P_{2}^{\prime}\right|$ of $L^{\prime}$ and $\ell$ is again the length $\left|P_{1} P_{2}\right|$ of $L$. Thus, to good approximation, the relative distortion $h / \ell$, is given by

$$
\frac{h}{\ell}=\frac{\left(y_{2}^{\prime}-y_{1}^{\prime}\right) x^{\prime}-\left(x_{2}^{\prime}-x_{1}^{\prime}\right) y^{\prime}+x_{2}^{\prime} y_{1}^{\prime}-x_{1}^{\prime} y_{2}^{\prime}}{\left(x_{2}-x_{1}\right)^{2}+\left(y_{2}-y_{1}\right)^{2}} \text {. }
$$

Hence, substituting via Eqs. (A12), (A13) and their analogues for $P_{1}^{\prime}(\theta=-\beta)$ and $P_{2}^{\prime}(\theta=\beta)$, we find that, to first order in $\Delta R / R$ and $\Delta \Theta / \Theta$,

$$
\frac{h}{\ell}=F(\alpha, \beta, \theta) \frac{\Delta R}{R}+G(\alpha, \beta, \theta) \beta \sin ^{2} \alpha \frac{\Delta \Theta}{\Theta},
$$

where

$$
\begin{aligned}
& F(\alpha, \beta, \theta)=\frac{1}{2} \frac{\sin \alpha}{\sin \beta}\left[\frac{\sin (\alpha+\beta) \sin (\alpha-\beta) \cos \theta}{\sin (\alpha-\theta) \cos \beta}+\sin (\alpha-\theta)-\sin (\alpha+\beta)-\sin (\alpha-\beta)\right],(A 18) \\
& G(\alpha, \beta, \theta)=\frac{\sin (\alpha+\beta) \sin (\alpha-\beta)}{\sin ^{2} \alpha \sin ^{2} 2 \beta \sin (\alpha-\theta)}\left[\sin (\alpha+\theta)-\cos 2 \beta \sin (\alpha-\theta)-\frac{\theta}{\beta} \sin 2 \beta \cos (\alpha-\theta)\right],
\end{aligned}
$$


which are Eqs. (4.1), (4.2), and (4.3), respectively.

\section{A.2 Error in length}

The relative error $\Delta \ell / \ell=\left(\ell^{\prime}-\ell\right) / \ell$ in the length $\ell$ of $L$, due to the errors $\Delta R$ in $R$ and $\Delta \Theta$ in $\Theta$, described above, is most easily found from

$$
\frac{\Delta \ell}{\ell}=\frac{\ell^{\prime}-\ell}{\ell}=\frac{\ell^{\prime 2}-\ell^{2}}{\left(\ell^{\prime}+\ell\right) \ell} \approx \frac{\ell^{\prime 2}-\ell^{2}}{2 \ell^{2}} \text {. }
$$

since, from Eqs. (A7) and (A8), we have that

$$
\begin{aligned}
& \left(x_{2}^{\prime}-x_{1}^{\prime}\right)=2 S^{\prime} \cos \beta^{\prime}=\ell \cos \alpha\left[1-\beta \tan \beta \frac{\Delta \Theta}{\Theta}\right], \\
& \left(y_{2}^{\prime}-y_{1}^{\prime}\right)=2 R^{\prime} \sin \beta^{\prime}=\ell \sin \alpha\left[1+\frac{\Delta R}{R}\right]\left[1+\beta \cot \beta \frac{\Delta \Theta}{\Theta}\right],
\end{aligned}
$$

and hence

$$
\ell^{\prime 2}=\ell^{2} \sin ^{2} \alpha\left[1+2 \frac{\Delta R}{R}+2 \beta \cot \beta \frac{\Delta \Theta}{\Theta}\right]+\ell^{2} \cos ^{2} \alpha\left[1-2 \beta \tan \beta \frac{\Delta \Theta}{\Theta}\right]
$$

correct to first order in $\Delta \Theta / \Theta$. Substituting this result into Eq. (A20) then yields

$$
\frac{\Delta \ell}{\ell}=\sin ^{2} \alpha\left(\frac{\Delta R}{R}+H(\alpha, \beta) \frac{\Delta \Theta}{\Theta}\right)
$$

correct to first order in $\Delta R / R$ and $\Delta \Theta / \Theta$, where

$$
H(\alpha, \beta)=\frac{\beta \sin (\alpha+\beta) \sin (\alpha-\beta)}{\sin ^{2} \alpha \sin \beta \cos \beta}=\frac{\beta}{\tan \beta}\left(\frac{1-\frac{\sin ^{2} \beta}{\sin ^{2} \alpha}}{1-\sin ^{2} \beta}\right),
$$

which are Eqs. (4.5) and (4.6).

\section{A.3 Error in area}

Consider the annular sector in an $x-y$ plane with respective inner and outer radii $R_{1}$ and $R_{2}$, and located between $\theta=\theta_{1}$ and $\theta=\theta_{2}$, with $\theta_{1}<\theta_{2}$. Let $\delta \theta=\theta_{2}-\theta_{1}$ be the angle that this sector subtends in this plane at the probe axis, 
and let $r=\left(R_{2}+R_{1}\right) / 2$ and $\delta r=\left(R_{2}-R_{1}\right)$. Then the sector area $A$ is exactly

$$
A=\frac{1}{2}\left(R_{2}^{2}-R_{1}^{2}\right)\left(\theta_{2}-\theta_{1}\right)=r \delta r \delta \theta .
$$

Now, when there is an error $\Delta R$ in the location of the probe axis and an error $\Delta \Theta$ in the total scanning angle $\Theta$, the image of the sector $A$ is the sector $A^{\prime}$ with respective inner and outer radii $R_{1}{ }^{\prime}=R_{1}+\Delta R$ and $R_{2}{ }^{\prime}=R_{2}+\Delta R$, located between $\theta_{1}^{\prime}=\theta_{1}(1+\Delta \Theta / \Theta)$ and $\theta_{2}^{\prime}=\theta_{2}(1+\Delta \Theta / \Theta)$, and subtending the angle $\delta \theta^{\prime}=\delta \theta(1+\Delta \Theta / \Theta)$ at the assumed probe axis location. Thus, $r^{\prime}=r+\Delta R$, $\delta r^{\prime}=\delta r$, and the distorted sector area $A^{\prime}$ is simply

$$
A^{\prime}=r^{\prime} \delta r^{\prime} \delta \theta^{\prime}=A\left(1+\frac{\Delta R}{r}\right)\left(1+\frac{\Delta \Theta}{\Theta}\right)=A+\Delta A,
$$

so that the relative error in area is exactly

$$
\frac{\Delta A}{A}=\left(1+\frac{\Delta R}{R}\right)\left(1+\frac{\Delta \Theta}{\Theta}\right)-1=\frac{\Delta R}{R}+\frac{\Delta \Theta}{\Theta}+\frac{\Delta R}{R} \frac{\Delta \Theta}{\Theta} .
$$

provided $R=r=\left(R_{2}+R_{1}\right) / 2$, which coincides with our definition of $R$ for the line joining the diagonally opposite corners $\left(R_{1}, \theta_{1}\right)$ and $\left(R_{2}, \theta_{2}\right)$ of the sector.

Now suppose that an area $A$ in an $x-y$ plane comprises $J$ annular sectors of areas $\delta A_{i j}$, with $r_{i}=\left(r_{2 j}+r_{1 j}\right) / 2, \delta r_{i}=\left(r_{2 j}-r_{1 j}\right)$, and $\delta \theta_{i}=\theta_{2 j}-\theta_{1 j}$, so that the corresponding distorted area $A^{\prime}=A+\Delta A$ comprises $J$ annular sectors of areas $\delta A_{j}^{\prime}$, with $r_{\mathrm{i}}=r_{\mathrm{i}}+\Delta R, \delta r_{\mathrm{j}}=\delta r_{\mathrm{i}}$, and $\delta \theta_{\mathrm{i}}^{\prime}=\delta \theta_{\mathrm{i}}(1+\Delta \Theta / \Theta)$, for $j=1, \ldots, J$. Then

$$
\begin{aligned}
& A=\sum \delta A_{i}=\sum r_{i} \delta r_{i} \delta \theta_{j}, \\
& A^{\prime}=\sum \delta A_{i}^{\prime}=\sum r_{i}^{\prime} \delta r_{i}^{\prime} \delta \theta_{i}^{\prime}=\sum\left(r_{i}+\Delta R\right) \delta r_{i} \delta \theta_{i}\left(1+\frac{\Delta \Theta}{\Theta}\right),
\end{aligned}
$$

and hence

$$
\Delta A=\Delta R\left(1+\frac{\Delta \Theta}{\Theta}\right) \sum \delta r_{i} \delta \theta_{i}+\frac{\Delta \Theta}{\Theta} \sum r_{i} \delta r_{i} \delta \theta_{i},
$$

so that 


$$
\frac{\Delta A}{A}=\frac{\Delta R}{R}\left(1+\frac{\Delta \Theta}{\Theta}\right)+\frac{\Delta \Theta}{\Theta}=\frac{\Delta R}{R}+\frac{\Delta \Theta}{\Theta}+\frac{\Delta R}{R} \frac{\Delta \Theta}{\Theta},
$$

where

$$
R=\frac{A}{A_{0}},
$$

with

$$
\begin{aligned}
& A=\sum r_{i} \delta r_{i} \delta \theta_{i} \rightarrow \iint_{A} r d r d \theta, \\
& A_{0}=\sum \delta r_{i} \delta \theta_{i} \rightarrow \iint_{A} d r d \theta,
\end{aligned}
$$

in the limit $\delta \theta_{j} \rightarrow d \theta, \delta r_{i} \rightarrow d r$, and $J \rightarrow \infty$. Hence, in this limit, Equations (A32)(A35) yield Eqs. (4.9) and (4.10), for the case of an arbitrary area $A$ in an $x-y$ plane. However, if $A$ is at an angle $\phi$ to this plane, let $A_{x y}=A \cos \phi$ and $\Delta A_{x y}=$ $\Delta A \cos \phi$ be the respective projections of $A$ and $\Delta A$ onto this plane, so that $\Delta A / A$ $=\Delta A_{x y} / A_{x y}$. The above analysis then shows that Eqs. (4.9) and (4.10) actually hold for an arbitrary area $A$ in any plane, i.e. for an arbitrary cross-sectional area.

\section{B. Variance of a manually determined edge location}

Consider the image of a white square in a black background. Each side of the square is then an edge separating white and black regions of the image. Suppose now that the image is not perfectly sharp, so that each edge actually comprises a gray transition zone with a width of $n$ pixels. Consider now a row or column of image pixels crossing an edge, and label the $n$ gray pixels as pixel 1 , pixel $2, \ldots$, pixel $n$. We now suppose that the coordinates of the edge are determined by manually selecting a cursor location within the edge image, with a uniform probability of selecting any one of the $n$ gray pixels, but no probability of

selecting a white or black pixel. Then the mean $\mu$ and variance $\sigma^{2}$ of the 
selected cursor location, in pixels, are clearly given by

$$
\begin{aligned}
& \mu=\frac{1}{n} \sum_{i=1}^{n} i=\frac{n+1}{2} \\
& \sigma^{2}=\frac{1}{n} \sum_{i=1}^{n}(i-\mu)^{2}=\frac{1}{n} \sum_{i=1}^{n} i^{2}-\mu^{2}=\frac{n^{2}-1}{12} .
\end{aligned}
$$

Hence, for a pixel size of $p$, the corresponding variance $\sigma_{p}^{2}$ is

$$
\sigma_{p}^{2}=\frac{n^{2}-1}{12} p^{2}
$$

Moreover, if the width $n p=w$ of the image edge is held constant, then in the limit $n \rightarrow \infty$ and $p \rightarrow 0$, i.e. for a continuous system, the above result becomes

$$
\sigma_{d}^{2} \rightarrow \frac{w^{2}}{12}
$$

which is equal to the quantization error for a location quantum of size $w$.

\section{C.1 Variance of the relative distortion}

By Eq. (4.14), the relative distortion $h / \ell$ is measured as

$$
\frac{h}{\ell}=\frac{y_{0}^{\prime}-\left(y_{2}^{\prime}+y_{1}^{\prime}\right) / 2}{x_{2}^{\prime}-x_{1}^{\prime}} \text {. }
$$

Hence, assuming that $y_{0}{ }^{\prime}, y_{1}{ }^{\prime}$, and $y_{2}{ }^{\prime}$ are each measured independently, with a common variance $\sigma_{y}{ }^{2}$, that $x_{1}{ }^{\prime}$ and $x_{2}{ }^{\prime}$ are each measured independently, with a common variance $\sigma_{\mathrm{x}}^{2}$ of similar magnitude to $\sigma_{\mathrm{y}}{ }^{2}$, and that the numerator $\left(y_{0}^{\prime}-\left(y_{1}{ }^{\prime}+y_{2}{ }^{\prime}\right) / 2\right)=h$ is much less than the denominator $\left(x_{2}^{\prime}-x_{1}{ }^{\prime}\right) \approx \ell$, then the variance $\sigma_{0}^{2}$ in the measured relative distortion $h / \ell$ is

$$
\begin{aligned}
\sigma_{0}^{2} & =\sum_{m=1}^{3}\left|\frac{\partial(h / \ell)}{\partial y_{m}}\right|^{2} \sigma_{y}^{2}+\sum_{n=1}^{2}\left|\frac{\partial(h / \ell)}{\partial x_{n}}\right|^{2} \sigma_{x}^{2} \\
& \approx 2 \frac{1}{\ell^{2}} \sigma_{y}^{2}+2 \frac{h^{2}}{\ell^{4}} \sigma_{x}^{2} \approx 2 \frac{\sigma_{y}^{2}}{\ell^{2}}
\end{aligned}
$$


which is Eq. (4.15).

\section{C.2 Variance of the relative error in length}

The relative error $\Delta \ell / \ell$ in $\ell$ is measured as

$$
\frac{\Delta \ell}{\ell}=\frac{x_{2}^{\prime}-x_{1}^{\prime}-\ell}{\ell}
$$

where the undistorted length $e$ is a known constant. Hence, assuming that $x_{1}{ }^{\prime}$ and $x_{2}{ }^{\prime}$ are each measured independently, with a common variance $\sigma_{x}{ }^{2}$, then the variance $\sigma^{2}$ in the measured relative error $\Delta \ell / \ell$ is

$$
\sigma_{1}^{2}=\sum_{n=1}^{2}\left|\frac{\partial(\Delta \ell / \ell)}{\partial x_{n}}\right|^{2} \sigma_{x}^{2}=2 \frac{\sigma_{x}^{2}}{\ell^{2}}
$$

which is Eq. (4.16).

\section{C.3 Variance of the relative error in area}

The image centroids $\left(x_{n}{ }^{\prime}, y_{n}{ }^{\prime}\right)$ (numbered counterclockwise) of three rows of three wires forming a parallelogram of $20-\mathrm{mm}$ base and $20-\mathrm{mm}$ height in the wire phantom were measured. In each case, the area $A^{\prime}$ of the eight-sided polygon image was then calculated from the exterior eight centroids via the formula (generalized from Spiegel, 1968):

$$
A^{\prime}=\frac{1}{2}\left(\left|\begin{array}{ll}
x_{1}^{\prime} & y_{1}^{\prime} \\
x_{2}^{\prime} & y_{2}^{\prime}
\end{array}\right|+\left|\begin{array}{ll}
x_{2}^{\prime} & y_{2}^{\prime} \\
x_{3}^{\prime} & y_{3}^{\prime}
\end{array}\right|+\cdots+\left|\begin{array}{cc}
x_{8}^{\prime} & y_{8}^{\prime} \\
x_{1}^{\prime} & y_{1}^{\prime}
\end{array}\right|\right),
$$

and the relative error $\triangle A / A$ in $A$ calculated as

$$
\frac{\Delta A}{A}=\frac{A^{\prime}-A}{A} \text {, }
$$

where the undistorted area $A$ is a known constant. Hence, assuming that the $x_{n}{ }^{\prime}$ are each measured independently, with a common variance $\sigma_{x}{ }^{2}$, and the $y_{n}^{\prime}$ are each measured independently, with a common variance $\sigma_{y}{ }^{2}$, we find that the variance $\sigma_{2}^{2}$ in the measured relative error $\triangle A / A$ is 


$$
\begin{aligned}
\sigma_{2}^{2} & =\sum_{n=1}^{8}\left|\frac{\partial(\Delta A / A)}{\partial x_{n}^{\prime}}\right|^{2} \sigma_{x}^{2}+\sum_{n=1}^{8}\left|\frac{\partial(\Delta A / A)}{\partial y_{n}^{\prime}}\right|^{2} \sigma_{y}^{2} \\
& =\sum_{n=1}^{8}\left|\frac{\partial A^{\prime}}{\partial x_{n}^{\prime}}\right|^{2} \frac{\sigma_{x}^{2}}{A^{2}}+\sum_{n=1}^{8}\left|\frac{\partial A^{\prime}}{\partial y_{n}^{\prime}}\right|^{2} \frac{\sigma_{y}^{2}}{A^{2}} \\
& =\sum_{n=1}^{8}\left|\frac{y_{n-1}^{\prime}-y_{n-1}^{\prime}}{2}\right|^{2} \frac{\sigma_{x}^{2}}{A^{2}}+\sum_{n=1}^{8}\left|\frac{x_{n+1}^{\prime}-x_{n-1}^{\prime}}{2}\right|^{2} \frac{\sigma_{y}^{2}}{A^{2}},
\end{aligned}
$$

where the subscripts are modulo 8 , so that $x_{0}=x_{8}$ and $x_{9}=x_{1}$.

Here, the base of the phantom parallelogram is parallel to the $x$-axis, formed by three wires uniformly spaced $1 \mathrm{~cm}$ apart, while each successive row of wires is $1 \mathrm{~cm}$ above and only slightly shifted laterally relative to the preceding row. The array of image centroids therefore approximates an almost square parallelogram of $2-\mathrm{cm}$ base, $2-\mathrm{cm}$ height, and area $A^{\prime} \approx A=4 \mathrm{~cm}^{2}$. Hence, locating $\left(x_{1}{ }^{\prime}, y_{1}{ }^{\prime}\right)$ in the lower left corner, and assuming the array to be approximately square, we find that

$$
\begin{aligned}
& \left|y_{3}^{\prime}-y_{1}^{\prime}\right|^{2}=\left|y_{7}^{\prime}-y_{5}^{\prime}\right|^{2} \approx 0 \\
& \left|y_{4}^{\prime}-y_{2}^{\prime}\right|^{2}=\left|y_{8}^{\prime}-y_{6}^{\prime}\right|^{2} \approx A / 4 \\
& \left|y_{5}^{\prime}-y_{3}^{\prime}\right|^{2}=\left|y_{1}^{\prime}-y_{7}^{\prime}\right|^{2} \approx A \\
& \left|y_{6}^{\prime}-y_{4}^{\prime}\right|^{2}=\left|y_{2}^{\prime}-y_{8}^{\prime}\right|^{2} \approx A / 4
\end{aligned}
$$

and

$$
\begin{aligned}
& \left|x_{3}^{\prime}-x_{1}^{\prime}\right|^{2}=\left|x_{7}^{\prime}-x_{5}^{\prime}\right|^{2} \approx A \\
& \left|x_{4}^{\prime}-x_{2}^{\prime}\right|^{2}=\left|x_{8}^{\prime}-x_{6}^{\prime}\right|^{2} \approx A / 4 \\
& \left|x_{5}^{\prime}-x_{3}^{\prime}\right|^{2}=\left|x_{1}^{\prime}-x_{7}^{\prime}\right|^{2} \approx 0 \\
& \left|x_{6}^{\prime}-x_{4}^{\prime}\right|^{2}=\left|x_{2}^{\prime}-x_{8}^{\prime}\right|^{2} \approx A / 4
\end{aligned}
$$

so that, to good approximation,

$$
\sigma_{2}^{2}=\frac{3}{4} \frac{\left(\sigma_{x}^{2}+\sigma_{y}^{2}\right)}{A}
$$

which is Eq. (4.18). 


\section{Determination of axis location}

A simple calibration phantom was used to determine the axis location $C$, measured in pixels from the top of each $2 D$ image, for each FOV setting of the ultrasound machine. This phantom consists of a square tube with one side serrated into four teeth by three milled slots, as illustrated in Fig. 6. Adjacent edges are uniformly separated by a known distance $D_{\mathrm{mm}}$ (in $\mathrm{mm}$ ) in the phantom, and a distance $D$ (in pixels) in the image. Each tooth is banded by a latex band of thickness $d_{\mathrm{mm}}$ (in $\mathrm{mm}$ ) and image width $d$ (in pixels), to form a set of eight parallel planes. The probe is placed in the middle slot, with its axis parallel to these planes, and the planes on both sides are imaged, rotating the probe $180^{\circ}$ between image acquisitions. The locations $A_{n}$ and $B_{n}$ of the line centroids on each side, also measured in pixels from the top of each $2 D$ image, are then used to determine $d_{\mathrm{mm}}$, the pixel size $p_{\mathrm{mm}}$ for each FOV, given by

$$
\rho_{m m}=\frac{D_{m m}}{D}=\frac{d_{m m}}{d}=\frac{D_{m m}+d_{m m}}{D+d}=\frac{D_{m m}-d_{m m}}{D-d},
$$

and finally the axis location $C$ for each FOV, as follows.

Since the physical separation of the latex planes with centroids $A_{1}$ and $B_{1}$ is $D_{\mathrm{mm}}-d_{\mathrm{mm}}$, while that of those with centroids $A_{2}$ and $B_{2}$ is $3 D_{\mathrm{mm}}+d_{\mathrm{mm}}$, and that of those with centroids $A_{3}$ and $B_{3}$ is $5 D_{\mathrm{mm}}-d_{\mathrm{mm}}$, the respective sums of the distances of these centroids from the axis, for each pair of phantom images, are

$$
\begin{aligned}
& \left(A_{1}-C\right)+\left(B_{1}-C\right)=D-d, \\
& \left(A_{2}-C\right)+\left(B_{2}-C\right)=3 D+d, \\
& \left(A_{3}-C\right)+\left(B_{3}-C\right)=5 D-d .
\end{aligned}
$$

Solving this set of equations for $C, D$, and $d$, we find

$$
\begin{aligned}
& C=\frac{\left(A_{1}+B_{1}\right)}{2}-\frac{(D-d)}{2}, \\
& D=\frac{\left(A_{3}+B_{3}\right)-\left(A_{1}+B_{1}\right)}{4},
\end{aligned}
$$




$$
d=\frac{2\left(A_{2}+B_{2}\right)-\left(A_{1}+B_{1}\right)-\left(A_{3}+B_{3}\right)}{4},
$$

so that, in particular,

$$
D+d=\frac{\left(A_{2}+B_{2}\right)-\left(A_{1}+B_{1}\right)}{2} .
$$

Hence, from Eqs. (D1), (D4), and (D5),

$$
d_{m m}=D_{m m} \frac{2\left(A_{2}+B_{2}\right)-\left(A_{3}+B_{3}\right)-\left(A_{1}+B_{1}\right)}{\left(A_{3}+B_{3}\right)-\left(A_{1}+B_{1}\right)},
$$

which is Eq. (4.19); while, from Eqs. (D1) and (D6),

$$
p_{m m}=\frac{2\left(D_{m m}+d_{m m}\right)}{\left(A_{2}+B_{2}\right)-\left(A_{1}+B_{1}\right)},
$$

which is Eq. (4.20); and, from Eqs. (D1) and (D3),

$$
C=\frac{\left(A_{1}+B_{1}\right)}{2}-\frac{\left(D_{m m}-d_{m m}\right)}{2 p_{m m}},
$$

which is Eq. (4.21).

\subsection{References}

1. Belohlavek M, Foley DA, Gerber TC, Kinter TM, Greenleaf JF, Seward JB. Three- and four-dimensional cardiovascular ultrasound imaging: $A$ new era for echocardiography. Mayo Clin Proc 68: 221-240; 1993.

2. Benson MC, Whang IS, Pantuck A, Ring K, Kaplan SA, Olsson CA, Cooner WH. Prostate specific antigen density: $A$ means of distinguishing benign prostatic hypertrophy and prostate cancer. J Urol 147: 815-816; 1992.

3. Benson MC, Whang IS, Olsson CA, MCMahon DJ, Cooner WH. The use of prostate specific antigen density to enhance the predictive value of intermediate levels of serum prostate specific antigen. J Urol 147: 817-821; 
1992.

4. Elliott TL, Downey DB, Tong S, McLean CA, Fenster A. Accuracy of prostate volume measurements in vitro using three-dimensional ultrasound. Academic Radiology 3: 401-406; 1996.

5. Fenster A, Downey DB. 3-D ultrasound imaging: A review. IEEE Eng. Med. Biol., 41-51; Nov./Dec. 1996.

6. Fenster A, Miller J, Tong S, Downey DB. Three-dimensional ultrasound imaging system. United States Patent 5,562,095 (October 8, 1996).

7. Greenleaf JF, Belohlavek M, Gerber TC, Foley DA, Seward JB. Multidimensional visualization in echocardiography: An introduction. Mayo Clin. Proc. 68: 213-220; 1993.

8. Rankin RN, Fenster A, Downey DB, Munk, PL, Levin MF, Vellet AD. Threedimensional sonographic reconstruction: Techniques and diagnostic applications. ARJ 161: 695-702; 1993.

9. Sehgal CM, Broderick GA, Whittington R, Gorniak RJT, Arger PH. Threedimensional US and Volumetric Assessment of the Prostate. Radiology 192: 274-278; 1994.

10. Tong S, Downey DB, Cardinal HN, Fenster A. A three-dimensional ultrasound prostate imaging system. Ultrasound Med. Biol. 22: 735-746; 1996.

11. Spiegel MR. Mathematical handbook of formulas and tables, Schaum's outline series. New York: McGraw-Hill; 1968 (p. 35).

12. Terris MK, MCNeal JE, Stamey TA. Estimation of prostate cancer volume by transrectal ultrasound imaging. J. Urol. 147: 855-857, 1992. 


\section{INTRA- AND INTER-OBSERVER VARIABILITY}

\subsection{Introduction}

Prostate-specific antigen (PSA) is currently recognized as the best single test for early diagnosis of prostate cancer. However, PSA is prostate-specific but not prostate cancer-specific, many different physical and pharmacological manipulations to the prostate may alter PSA level, and the overlap of serum PSA levels in different conditions makes the specificity of PSA testing less than ideal (Arcangeli CG et al. 1997). PSA density, calculated by dividing the serum PSA level by the prostate volume, has been suggested as one possible method to improve the specificity of serum PSA testing (Benson et al. 1992). Thus, the accurate estimation of prostate volume becomes important.

Conventionally, with 2D ultrasound, prostate volume estimation is done by measuring the height $(H)$, width $(W)$, and length $(L)$ of the prostate from two selected orthogonal views (e.g. transverse and sagittal), and estimating the prostate volume $V$ as that of the corresponding ellipsoid, i.e. $V=(\pi / 6) H W L$. This is called the HWL method. The measurement is performed by using position tracking device on the $2 \mathrm{D}$ images of the prostate on the ultrasound machine during the patient examination. This method has several drawbacks: the prostate is not ellipsoidal; consequently, the choice of which three chords are to be used to measure $H, W$, and $L$ in a given set of images is not clear-cut, and largely dependent on observer preference, leading to high inter-observer variability in estimating $V$. Moreover, even for a single observer with a single set of images, the choice is still somewhat arbitrary, leading to high intra-observer variability in estimating $V$. Furthermore, since each $2 D$ image represents a thin slice of the patient's anatomy in a particular location and orientation, it is difficult to select the same two image planes in a subsequent examination, compounding the difficulty of monitoring any changes in $V$ over the course of time, e.g. as a result of radiation and antiandrogen therapy. Nevertheless, the HWL method is one of the best methods currently available for estimating $V$ from only two 
orthogonal 2D views of the prostate (Terris et al. 1992).

To overcome these difficulties, we have developed a 3D ultrasound system for imaging the prostate (Fenster et al. 1996; Tong et al. 1996). The system is comprised of a conventional ultrasound machine and transrectal ultrasound prostate probe; a custom-built assembly to hold and rotate the probe under microcomputer control; a microcomputer with an 8-bit video frame-grabber; and, software for $2 \mathrm{D}$ image data acquisition and 3D image reconstruction. The prostate probe is rotated about its axis by a computer-controlled motor, at a constant rate. At pre-defined intervals, a region of interest (ROI) within the $2 D$ image is digitized and stored in computer memory. With a side-firing ultrasound probe, a typical scan consists of $1002 \mathrm{D} \mathrm{B}$-mode images covering a total angle of $80^{\circ}$. If an end-firing probe is used, a typical scan consists of $2002 \mathrm{D} \mathrm{B-mode} \mathrm{images} \mathrm{covering}$ a total angle of $200^{\circ}$. After acquisition, the scan data is reconstructed into a 3D volume image for immediate or later review by the radiologists. The $3 \mathrm{D}$ volume image of the prostate can be viewed interactively on a computer, using commercially available or custom-designed 3D visualization software (Fenster et al. 1995; Tong et al 1996), to view any cross-sectional "slice" of the prostate, in any orientation, via "multi-slicing texture mapping".

With 3D ultrasound, prostate volume $V$ can be estimated via manual planimetry. In this method, the 30 volume image of the prostate is "sliced" in the computer into a series of uniformly-spaced, parallel $2 D$ images, and the crosssectional area of the prostate in each slice is then manualiy outlined interactively on a computer with a computer mouse device. The sum of these areas, multiplied by the slice thickness, then provides an accurate estimate of $V$ (as demonstrated with water-filled balloons in Chapter 2 and with cadaver prostates in Chapter 3). Henceforth, we will refer to this method as the 3D US method. This method is illustrated by Figure 5.1 , which shows the manually drawn outline of the prostate in a sample cross-sectional $2 D$ image "sliced" from a $3 D$ ultrasound image of the prostate. 


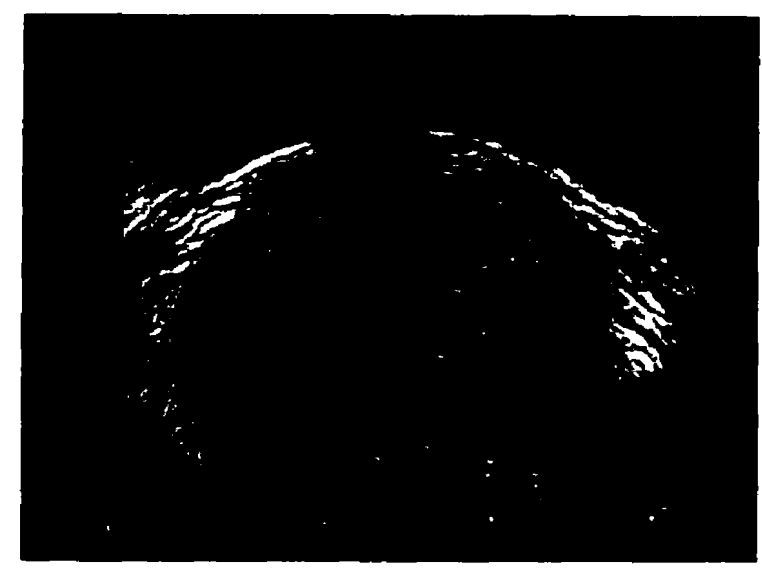

Figure 5.1 Manually drawn outline of the prostate in a sample cross-sectional 2D image "sliced" from a 3D ultrasound image of the prostate.

Previous studies have shown that the 3D US method provides better accuracy, precision, and reproducibility in volume estimation than the HWL method (Chang et al. 1997; Tong et al. 1996; Elliot et al. 1996; Riccabona et al. 1995, 1996; Gilja OH 1994; Terris MK 1991, 1992).

In one such study, Riccabona et al. (1995) reported on ultrasound volume measurements, in vitro, of 30 water-filled balloons, ranging in shape from spherical to irregular, and in size from $23 \mathrm{ml}$ to $2400 \mathrm{ml}$. The results showed that the HWL method had a mean error of $13.7 \% \pm 10.1 \%$, while the $3 D$ US method had a mean error of $2.2 \% \pm 2.9 \%$. Moreover, the correlation coefficients of linear regressions of actual vs measured volumes also showed the superiority of 3D US over HWL in all shape classes, but especially for the irregular class.

Subsequently, Riccabona et al. (1996) reported on ultrasound volume measurements, in vivo, of 50 normal urinary bladders. Here, the results showed that the HWL method had a mean error of $27.5 \% \pm 17.8 \%$, while the 3D US method had a mean error of $5.6 \% \pm 3.8 \%$.

Also, Chang et al. (1997) has reported on the reproducibility of 3D US vs HWL in the assessment of fetal liver volume, in vivo, for 30 fetuses, ranging in gestational age from 20 to 30 weeks. Intra-observer reproducibility was 
determined from repeated volume measurements by one observer, while inter-observer reproducibility was determined from independent measurements by two observers. In both cases, 3D US was superior to HWL. The standard deviation of intra-observer volume measurements was $8.46 \mathrm{~cm}^{3}$ for $\mathrm{HWL}$, and $2.15 \mathrm{~cm}^{3}$ for $3 D$ US, while the standard deviation of inter-observer volume measurements was $6.60 \mathrm{~cm}^{3}$ for HWL and $2.02 \mathrm{~cm}^{3}$ for 3D US.

In this chapter, the analysis of variance (ANOVA) technique described by Eliasziw et al. (1994) and implemented by Mitchell et al. (1996) is used to assess the intra- and inter-observer variability of prostate volume measurements made via the HWL and 3D US methods. In particular, we respectively use the intra- and inter-observer reliability coefficients $\rho_{\text {intra }}$ and $\rho_{\text {inter }}$ to characterize the consistency and reproducibility of volume measurements made by the same and different observers, and the intra- and inter-observer standard errors of measurement SEM intra and SEM inter to characterize the variability of volume measurements made by the same and different observers, both as absolute and percentage values. These latter quantities can also be expressed in terms of the minimum volume changes $\Delta V_{\text {intra }}$ and $\Delta V_{\text {inter }}$ that can be detected with a given confidence level in successive measurements by the same and different observers, respectively, and these are also reported in both absolute and percentage terms for a confidence level of $95 \%$. Using the two sets of measured parameters, one for each method, we then compare HWL and 3D US for measuring prostate volume.

\subsection{Materials and Methods}

\subsubsection{Study design}

Eight observers participated in this study. Half of these are experienced radiologists at the London Health Sciences Centre, and the other half are technicians or graduate students from the Imaging Research Laboratories, Robarts Research Institute. Dr. D. B. Downey provided two sessions of training, 
two hours each session, to the non-radiologists (the technicians and graduate students) on how to make prostate volume measurement from a 3D ultrasound image. Fifteen prostate images, scanned in vivo, and reconstructed and measured using software developed at the Imaging Research Laboratories, were used in this study. The volume of each prostate was measured four times by each observer, twice via the HWL method, using transverse and sagittal crosssections of the $3 D$ volume image to measure $H, W$, and $L$, and twice via the $3 D$ US method, using manual planimetry with an interslice spacing of $4 \mathrm{~mm}$. The rationale for choosing these numbers of observers, prostates, and repeated measurements will be discussed in more detail in Section 5.4.

Working independently, each observer was required to measure the transverse, sagittal, and antero-posterior dimensions of a selected prostate, and manually outline its cross-section in 7 to 14 image "slices" (depending on the prostate size) but to do no calculations, in each session, at a rate of no more than one session per week. In each series of 15 sessions, the prostate order was individually randomized for each observer, in order to minimize any memory of the measurements from one series to the next. Due to this protocol, it took more than 30 weeks ( 7 months) to complete the two series of measurements made for this study.

Ideally, the HWL method should be performed on $2 D$ images from a conventional ultrasound machine, as part of a normal examination. However, examining each patient 16 times is neither practical nor ethically acceptable. Hence, we performed the HWL measurements on sagittal and transverse $2 D$ cross-sections of the 3D US image, as a practical and acceptable substitute.

\subsubsection{Standard deviations of volume measurements}

Table 5.1 is the data layout for the repeated measurement study. $V_{i \mathrm{ik}}$ is the volume of the $i$ th prostate as measured by the $j$ th observer for the $k$ th time, where the prostate $i=1, \ldots p$; the observer $j=1, \ldots o$; and the measurement $k=1, \ldots m$. In this study, the number of prostates $p=15$, the number of 
observers $0=8$, and the number of measurements per prostate per observer $m=2$.

Table 5.1 Data layout for repeated measurements of prostate volumes. $V_{i j k}$ is the volume of the $i$ th prostate measured by the $j$ th observer for the $k$ th time, where $i=1, \ldots, p ; j=1, \ldots, 0 ;$ and $k=1, \ldots, m$. Here, $p=15, o=8$, and $m=2$.

\begin{tabular}{|c|c|c|c|c|c|c|c|c|c|c|c|c|}
\hline & \multicolumn{6}{|c|}{$k=1$} & \multicolumn{6}{|c|}{$k=2$} \\
\hline & 1 & 2 & $\ldots$ & $j$ & $\ldots$ & c & 1 & 2 & $\ldots$ & j & $\ldots$ & 0 \\
\hline 1 & & & & & & & & & & & & \\
\hline 2 & & & & & & & & & & & & \\
\hline$\ldots$ & & & & & & & & & & & & \\
\hline$i$ & & & & $V_{i j k}$ & & & & & & & & \\
\hline$\ldots$ & & & & & & & & & & & & \\
\hline$p$ & & & & & & & & & & & & \\
\hline
\end{tabular}

For both the HWL and 3D US methods, the mean measured prostate volume is plotted vs prostate number in a bar graph, using one-sided error bars to indicate the standard deviations of the measured volumes. Also, the relative standard deviations are plotted vs prostate number in a bar graph, and vs prostate volume in a scatter plot.

\subsubsection{Systematic and random observer errors}

Since the true prostate volumes are unknown, the mean volumes measured by the radiologists (observers 1 to 4 ) with the 3D US method are considered to be the true volumes, used here as a reference in order to calculate relative errors, i.e. errors normalized to unit prostate volume, in a consistent manner, and to assess the systematic errors of individual observers.

For both the HWL and 3D US methods, each observer's systematic error was estimated as the mean relative error of their volume estimates, i.e. the mean percentage difference between the measured and true prostate volumes described above. Thus, the average systematic error of the radiologists 
(observers 1 to 4) with the 3D US method is normalized to zero here. Similarly, each observer's random error was estimated as the mean relative standard deviation of their volume estimates, i.e. the standard deviations expressed as a percentage of these true prostate volumes, for both methods. Averaging these individual systematic and random errors over all 8 observers then yielded the mean systematic and random observer errors, respectively. Also, in order to assess any differences between radiologists (observers 1 to 4 ) and nonradiologists (observers 5 to 8 ), the averages over these subgroups of observers were also determined.

\subsubsection{Analysis of Variance}

Using the terminology of Eliasziw et al. (1994) and Mitchell et al. (1996), each prostate volume measurement can be described via the following model:

$$
V_{i \mathrm{ik}}=\mu+p_{i}+a_{i}+p a_{i j}+e_{i k}
$$

where $V_{i j k}$ is the volume of the $i$ th prostate as measured by the $j$ th observer for the $k$ th time; $\mu$ is the overall mean volume; $p$ is effect of prostate $i$ on the volume measurement; $o_{i}$ is the effect of observer $j$ on the volume measurement; $p o_{i j}$ is the effect of the interaction between prostate $i$ and observer $j$ on the volume measurement; and $e_{i \mathrm{ik}}$ is the intra-observer random error in $V_{\mathrm{ijk}}$. The effects $p_{1} q_{\mathrm{i}}$, $p o_{i j}$, and $e_{i j k}$ are assumed to have zero means and variances of $\sigma_{p}{ }^{2}, \sigma_{0}{ }^{2}, \sigma_{p o}{ }^{2}$, and $\sigma_{e}{ }^{2}$, respectively, so that the total variance of repeated measurements is given by:

$$
\sigma^{2}=\sigma_{p}^{2}+\sigma_{0}^{2}+\sigma_{\rho 0}^{2}+\sigma_{e}^{2} .
$$

The analysis of variance (ANOVA) software developed by J. R. Mitchell for analysis of the variability of multiple sclerosis lesion volumes was adapted to this study. Table 5.2 shows the ANOVA table used to estimate the components of the variance (Mitchell et al. 1996). Here, the number of prostates $p=15$; the number of observers $0=8$; and the number of repeated measurements $m=2$. 
Table 5.2 Analysis of variance (ANOVA) table for repeated measurement experiments used to study intra- and inter-observer reliability and variability simultaneously. Here, the number of prostates $p=15$; the number of observers $o=8$; and the number of repeated measurements $m=2$.

\begin{tabular}{cccc}
\hline $\begin{array}{c}\text { Source of } \\
\text { Variance }\end{array}$ & $\begin{array}{c}\text { Degrees of } \\
\text { Freedom }\end{array}$ & $\begin{array}{c}\text { Observed } \\
\text { Mean Squares }\end{array}$ & $\begin{array}{c}\text { Expected } \\
\text { Mean Squares }\end{array}$ \\
\hline Prostate & $p-1$ & $\mathrm{MS}_{\mathrm{p}}$ & $m o \sigma_{\mathrm{p}}{ }^{2}+m \sigma_{p o}{ }^{2}+\sigma_{\mathrm{e}}{ }^{2}$ \\
Observer & $0-1$ & $\mathrm{MS}_{0}$ & $m p \sigma_{0}{ }^{2}+m \sigma_{p o}{ }^{2}+\sigma_{\mathrm{e}}{ }^{2}$ \\
Observer $\times$ Prostate & $(p-1)(0-1)$ & $\mathrm{MS}_{\mathrm{po}}$ & $m \sigma_{p o}{ }^{2}+\sigma_{\mathrm{e}}{ }^{2}$ \\
Intra-observer & $p o(m-1)$ & $\mathrm{MS}_{\mathrm{e}}$ & $\sigma_{\mathrm{e}}{ }^{2}$ \\
\hline
\end{tabular}

Equating the expressions in Table 5.2 for the observed and expected mean squares, we can solve for the variance components $\sigma_{\rho}{ }^{2}, \sigma_{0}{ }^{2}, \sigma_{\infty}{ }^{2}$, and $\sigma_{e}{ }^{2}$ to find:

$$
\begin{aligned}
& \sigma_{p}^{2}=\left(M S_{p}-M S_{p o}\right) /(m o) \\
& \sigma_{0}^{2}=\left(M S_{0}-M S_{p o}\right) /(m p) \\
& \sigma_{p o}{ }^{2}=\left(M S_{p o}-M S_{e}\right) / m \\
& \sigma_{e}{ }^{2}=M S_{e}
\end{aligned}
$$

Then, following Mitchell et al. (1996), these components can be used to calculate the intra- and inter-observer reliability coefficients $\rho_{\text {intra }}$ and $\rho_{\text {inter: }}$

$$
\begin{aligned}
& \rho_{\text {intra }}=\left(\sigma_{p}^{2}+\sigma_{0}^{2}+\sigma_{p o}{ }^{2}\right) / \sigma^{2}=1-\left(\sigma_{e}{ }^{2} / \sigma^{2}\right) \\
& \rho_{\text {inter }}=\sigma_{p}{ }^{2} / \sigma^{2}=1-\left(\sigma_{0}^{2}+\sigma_{p o}{ }^{2}+\sigma_{e}{ }^{2}\right) / \sigma^{2}
\end{aligned}
$$

These coefficients vary between 0 and 1 , with higher values indicating greater consistency and reproducibility in repeated prostate volume measurements. Also, the intra- and inter-observer standard errors of measurement, SEM intra and $S_{\text {SEM }}$ inter, which respectively characterize the variability between successive volume measurements by the same and different observers, are given by:

$$
S E M_{\text {intra }}=\sqrt{ } \sigma_{e}^{2}
$$




$$
S E M_{\text {inter }}=\sqrt{ }\left(\sigma_{o}^{2}+\sigma_{p o}^{2}+\sigma_{e}^{2}\right)
$$

Then, following Eliasziw et al. (1994), the minimum volume change $\Delta V$ that can be detected with a given confidence level in two successive measurements by the same and different observers, respectively, can be calculated as:

$$
\begin{aligned}
& \Delta V_{\text {intra }}=z_{\alpha} \times \sqrt{ } 2 \times S E M_{\text {intra }} \\
& \Delta V_{\text {inter }}=z_{\alpha} \times \sqrt{ } 2 \times S E M_{\text {inter }}
\end{aligned}
$$

where $z_{u}$ is the standard normal deviate exceeded on either side of the mean with total (two-tailed) probability $\alpha$. For a confidence level of $95 \%$, i.e. $\alpha=0.05$, $z_{\alpha}=1.96$, and this value of $z_{\alpha}$ is adopted here.

The $\rho$, SEM, and $\Delta V$ parameters described above were calculated for both the HWL and 3D US methods, and the results are tabulated below. However, applying the ANOVA to different subsets of the 15 prostates, it was found that the SEM and $\Delta V$ parameters, which have dimensions of volume, varied according to the average volume of the subset. Therefore, for each method, each volume measurement was first normalized by the mean volume of that prostate, as measured by that method, and the ANOVA reapplied, to obtain relative, i.e. percentage, SEM and $\Delta V$ parameters that are independent of prostate volume. These are also tabulated below for both methods.

The statistical significance of the difference between the SEMs for the two methods was tested using the $t$-test, where the appropriate value of statistic $t$ is calculated as (Mitchell et al. 1996):

$$
t=\left(\mathrm{SEM}^{2}{ }_{\mathrm{HWL}}-\mathrm{SEM}^{2}{ }_{3 \mathrm{DUS}}\right) / \sqrt{ }\left(\operatorname{var}\left(\mathrm{SEM}^{2}{ }_{\mathrm{HWL}}\right)+\operatorname{var}\left(\mathrm{SEM}^{2}{ }_{3 \mathrm{DUS}}\right)\right) \text {, }
$$

where $\operatorname{var}\left(\mathrm{SEM}^{2}\right)$, the variance in the $S E M^{2}$, is given by

$$
\operatorname{var}\left(\mathrm{SEM}^{2}\right)=2 \mathrm{SEM}^{4} / v(\mathrm{SEM}) \text {, }
$$

and $v($ SEM $)=p \times 0 \times(m-1)$ is the number of degrees of freedom in the SEM. Here, for $p=15$ prostates, $0=8$ observers, and $m=2$ measurements per prostate per observer, $v=120$. 


\subsection{Results}

\subsubsection{Standard deviations of volume measurements}

Figure 5.2 shows a bar graph of measured prostate volume vs prostate number for both the HWL and 3D US methods. The height of each bar represents the mean of the 16 measurements made by all 8 observers for each method, while each error bar represents their standard deviation. The standard error of the mean is thus $\sqrt{16}$ or 4 times smaller than these error bars. From this graph, we see that the HWL volumes and standard deviations are both systematically larger than their 3D US counterparts. From this data, we find the mean and standard deviation of the measured prostate volumes to be $76.3 \pm$ $13.4 \mathrm{~cm}^{3}$ and $69.2 \pm 7.7 \mathrm{~cm}^{3}$ for the HWL and 3D US methods, respectively. Moreover, for both methods, the standard deviation varies as the prostate size.

Therefore, we have plotted the relative standard deviation vs prostate number in Figure 5.3 and vs prostate volume in Figure 5.4, for the two methods. From these plots, we see that the relative standard deviation for the HWL method is significantly larger than for the 3D US method, with a mean and standard deviation of $0.186 \pm 0.047$ for the HWL method and $0.108 \pm 0.022$ for the 3D US method. The difference has a $p$-value of $p<0.001$ in a paired $t$-test.

In Figure 5.4, a horizontal solid line indicates the mean value of the relative standard deviation, and parallel dashed lines the mean value plus or minus one standard deviation, for each method. For both methods, all 15 plotted points lie within 1.5 standard deviations of the mean, and there is no significant correlation with prostate volume. Since these relative standard deviations include both intra- and inter-observer components, we may reasonably infer that these components also have no significant correlation with prostate volume. 


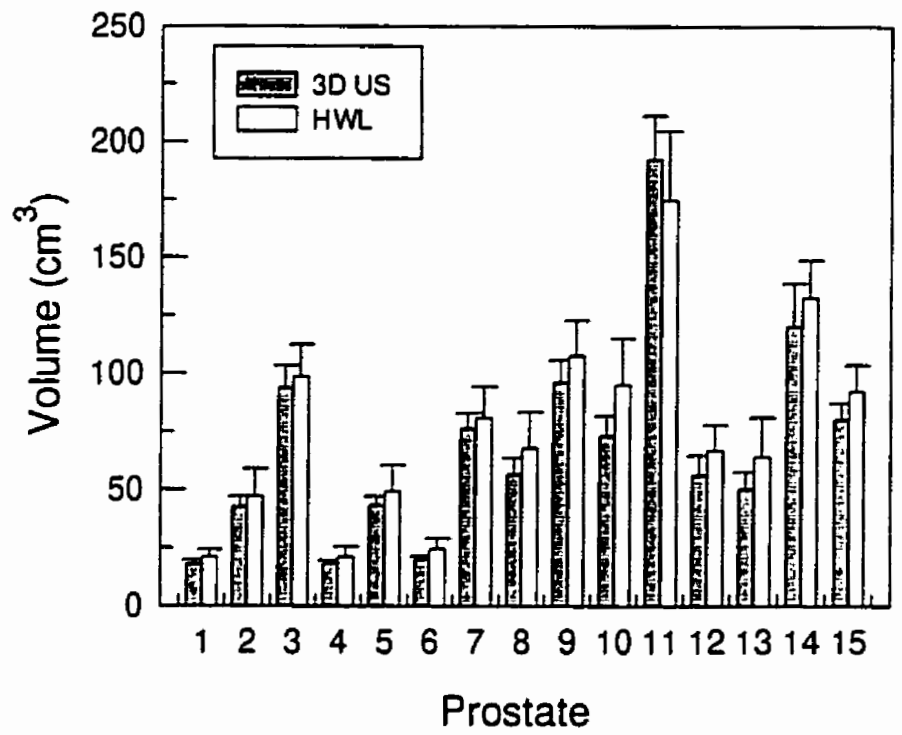

Figure 5.2 Measured prostate volume vs prostate number for the HWL and 3D US methods. Each error bar is the standard deviation of 16 measurements. The mean \pm standard deviation of the measured prostate volumes is $76.3 \pm 13.4$ $\mathrm{cm}^{3}$ for the HWL method and $69.2 \pm 7.7 \mathrm{~cm}^{3}$ for the $3 \mathrm{D}$ US method.

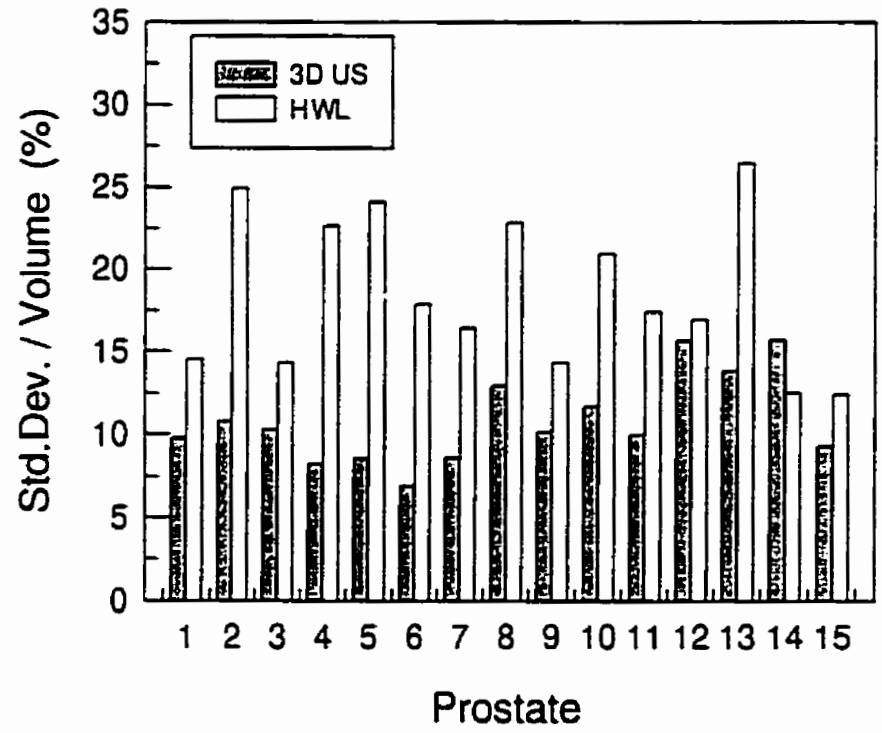

Figure 5.3 Relative standard deviation vs prostate number for the HWL and 3D US methods. The mean \pm standard deviation of these values is $18.6 \% \pm 4.7 \%$ for the HWL method and $10.8 \% \pm 2.6 \%$ for the $3 D$ US method. 

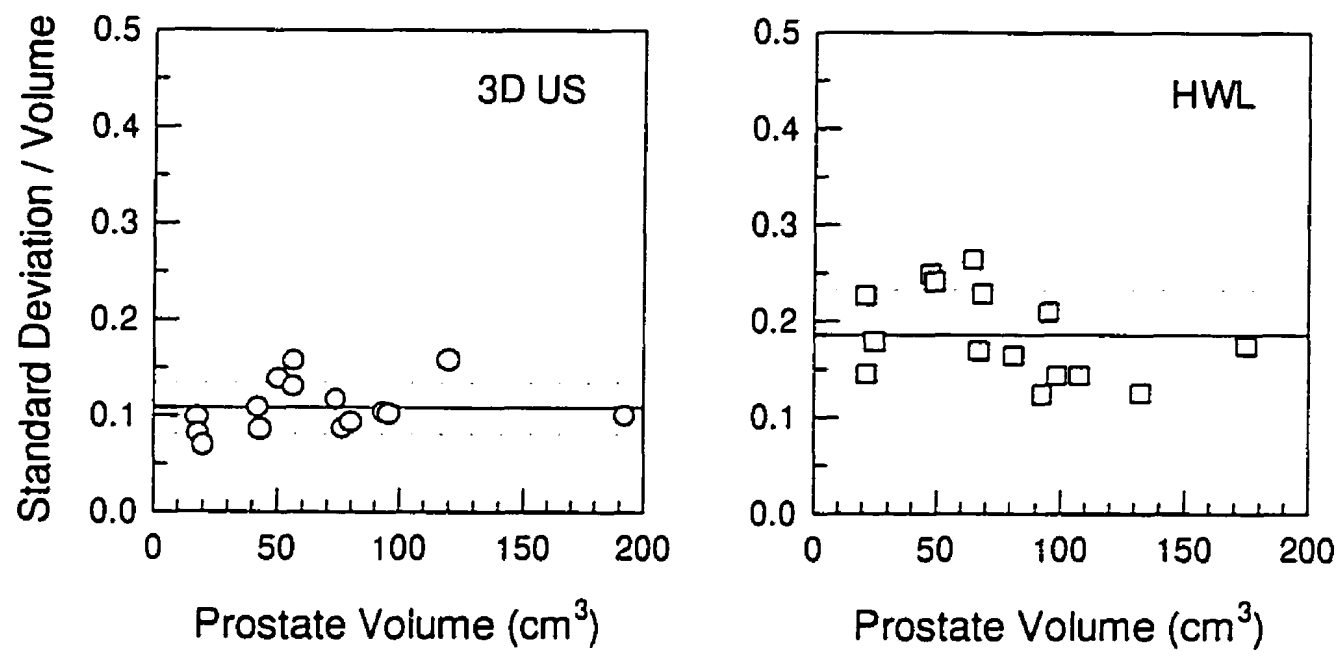

Figure 5.4 Relative standard deviation vs prostate volume for the HWL and $3 D$ US methods. The mean \pm standard deviation of these values is $0.186 \pm 0.047$ for the HWL method and $0.108 \pm 0.022$ for the 3D US method. For each method, the solid horizontal lines indicate the mean values, and the dashed lines the mean values plus or minus one standard deviation.

\subsubsection{Systematic and random observer errors}

In Figure 5.5, we have plotted systematic error vs observer number for the HWL and 3D US methods. As described above, the "true" prostate volume is here estimated as the mean volume measured by radiologists (observers 1 to 4 ) with the 3D US method. Thus, a bar above (below) the zero line indicates that an observer tends to over- (under-) estimate the prostate volume, in comparison to an average radiologist using the 3D US method. Here, the error bars indicate the standard error in the systematic error for each observer.

The data plotted in Figure 5.5 is analyzed in Table 5.3. This analysis shows that, in comparison to radiologists (observers 1 to 4), non-radiologists (observers 5 to 8 ) tend to over-estimate prostate volume significantly with HWL $(p<0.01)$, but negligibly with 3D US ( $p>0.5)$. 


\section{Systematic Observer Error}

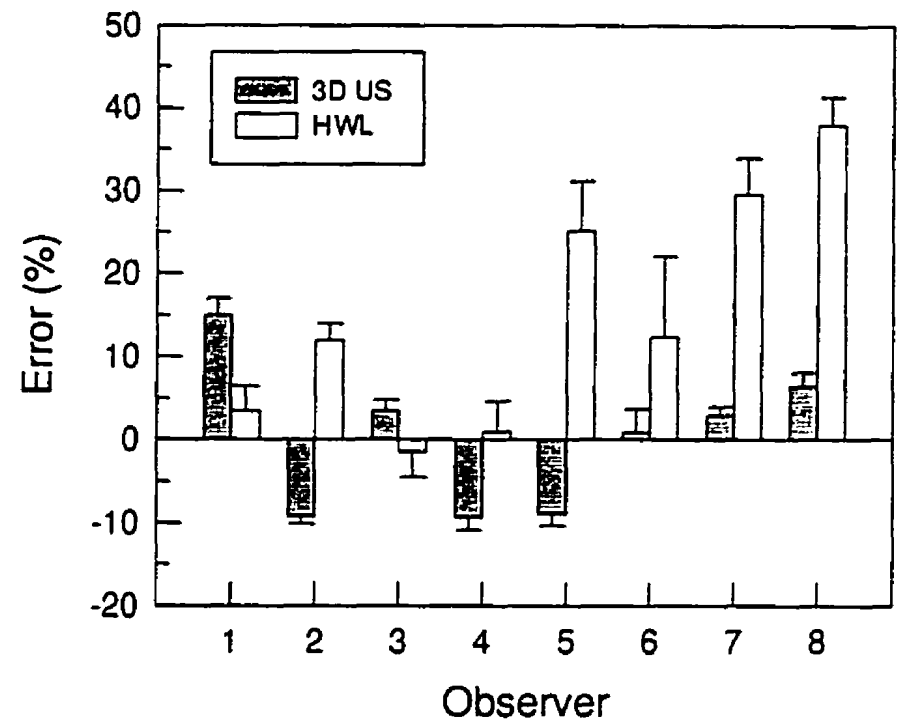

Figure 5.5 Systematic observer error vs observer number, for the HWL and 3D US methods. The "true" volume of each prostate was estimated as the average volume measured by radiologists (observers 1 to 4) with the 3D US method. The error bars indicate the standard error in the systematic error for each observer.

Table 5.3 Mean and standard deviation of systematic observer error.

\begin{tabular}{ccc}
\hline & HWL (\%) & 3D US (\%) \\
\hline Radiologists & $3.7 \pm 5.9$ & $0.0 \pm 11.7$ \\
Non-Radiologists & $26.3 \pm 10.6$ & $0.3 \pm 6.6$ \\
All & $15.0 \pm 14.4$ & $0.2 \pm 8.8$ \\
\hline \hline
\end{tabular}

In Figure 5.6, we have plotted random observer error vs observer number, as a percentage of the "true" prostate volume, for the HWL and 3D US methods. From this graph, we see that, with each method, the random errors of nonradiologists and radiologists are comparable, and that the random errors of both 
groups are significantly lower with the 3D US method than the HWL method $(p<0.01)$.

The data plotted in Figure 5.6 is analyzed in Table 5.4. This analysis shows that, on average, using the 3D US method reduces random error by about a factor of three, in comparison to the HWL method.

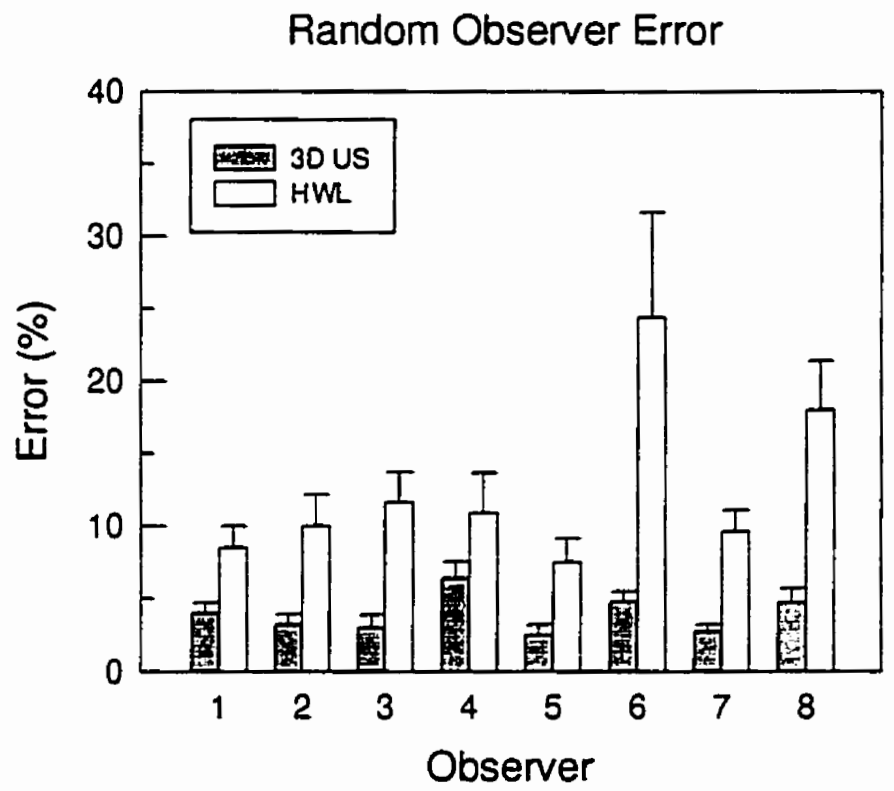

Figure 5.6 Random observer error vs observer number, as a percentage of prostate volume. The error bars indicate the standard error in the random error for each observer. Observers 1.4 are radiologists, and 5-8 are non-radiologists.

Table 5.4 Mean and standard deviation of random observer error.

\begin{tabular}{ccc}
\hline & HWL (\%) & 3D US (\%) \\
\hline Radiologists & $10.3 \pm 1.3$ & $4.2 \pm 1.6$ \\
Non-Radiologists & $14.9 \pm 7.8$ & $3.7 \pm 1.2$ \\
All & $12.6 \pm 5.7$ & $3.9 \pm 1.3$ \\
\hline
\end{tabular}




\subsubsection{Analysis of variance}

In Table 5.5, we tabulate the intra- and inter-observer reliability coefficients $p_{\text {intra }}$ and $p_{\text {inter }}$ found by applying the analysis of variance (ANOVA) technique to the prostate volume measurements made by the HWL and $3 D$ US methods. From this table, we see that the respective values of $\rho_{\text {intra }}$ and $\rho_{\text {inter }}$ are 0.93 and 0.87 for the HWL method, and 0.99 and 0.96 for the 3D US method.

Table 5.5 The intra- and inter-observer reliability coefficients $\rho_{\text {intra }}$ and $\rho_{\text {inter }}$ from an analysis of variance (ANOVA) of prostate volume measurements made by the HWL and 3D US methods.

\begin{tabular}{ccc}
\hline & HWL & 3D US \\
\hline$\rho_{\text {intra }}$ & 0.93 & 0.99 \\
$\rho_{\text {inter }}$ & 0.87 & 0.96 \\
\hline \hline
\end{tabular}

In Table 5.6, we tabulate the standard errors of measurement SEM $_{\text {intra }}$ and SEM $M_{\text {inter }}$ from the same analysis, as well as $\Delta V_{\text {intra }}$ and $\Delta V_{\text {inter, the corresponding }}$ minimum volume changes detectable at the $95 \%$ level of confidence, for each method. However, similar analyses performed on different subsets of the original 15 prostates showed that these parameters (which have dimensions of volume) vary according to the average volume of the subset. Accordingly, for each method, each volume measurement was normalized by the mean volume of that prostate, as measured by that method by all observers, and an ANOVA performed on the resulting data. This yielded the percentage values listed in Table 5.7, which correspond to the absolute values listed in Table 5.6. As it is seen in Table 5.3 that measurements from non-radiologists using the HWL method tend to have large systematic errors. To avoid these errors skewing the results of SEM and $\Delta V$, in Table 5.6 and Table 5.7 only measurements from radiologists were included for the analysis of the HWL method. 
We remark that, since $V$ varies as the cube of linear prostate size, the minimum detectable percentage changes in the linear prostate dimensions are less than a third of the $\Delta V$ values shown here, so that these values are not as excessive as they initially appear to be.

Table 5.6 The standard errors of measurement SEM intra and SEM inter from an analysis of variance (ANOVA) of prostate volume measurements made by the $H W L$ and $3 D$ US methods, and the corresponding minimum volume changes $\Delta V_{\text {intra }}$ and $\Delta V_{\text {inter }}$ detectable at the $95 \%$ level of confidence.

\begin{tabular}{ccc}
\hline & $\mathrm{HWL}\left(\mathrm{cm}^{3}\right)$ & 3D US $\left(\mathrm{cm}^{3}\right)$ \\
\hline SEM & 9.5 & 3.6 \\
SEM & 11.6 & 9.6 \\
\hline$\Delta V_{\text {intra }}$ & 26.3 & 10.0 \\
$\Delta V_{\text {inter }}$ & 32.1 & 26.6 \\
\hline
\end{tabular}

Table 5.7 The standard errors of measurement SEM intra and SEM inter from an analysis of variance (ANOVA) of normalized prostate volume measurements by the HWL and 3D US methods, and the corresponding minimum volume changes $\Delta V_{\text {intra }}$ and $\Delta V_{\text {inter }}$ detectable at the $95 \%$ level of confidence. For each method, each volume measurement was normalized by the mean volume of that prostate, as measured by that method by all observers, before analysis.

\begin{tabular}{ccc}
\hline & HWL (\%) & 3D US (\%) \\
\hline SEM & 11.5 & 5.1 \\
SEM & 13.5 & 11.4 \\
\hline$\Delta V_{\text {intra }}$ & 31.9 & 14.1 \\
$\Delta V_{\text {inter }}$ & 37.4 & 31.6 \\
\hline \hline
\end{tabular}




\subsection{Discussion and Conclusions}

\subsubsection{Study design}

In the design of a variability study, an important consideration is the sample size: specifically, the number of observers to be recruited, the number of prostates to be measured, and the number of measurements of each prostate to be performed by each observer. If the sample size is too small, it may be difficult to demonstrate that the reliability $\rho$ exceeds a specified critical value $\rho_{0}$. On the other hand, if the sample size is too big, resources are wasted. However, although the required sample size depends on $p$ (among other factors), this is unknown until after the study has been completed. Thus, a trial study is needed to estimate $\rho$.

Donner and Eliasziw (1987) and Eliasziw et al. (1994) provide guidelines for the planning of intra-observer reliability studies, with graphs showing the numbers of subjects and repeated measurements that provide $80 \%$ power for testing the hypothesis $H_{0}: \rho \leq \rho_{0}$ versus the hypothesis $H_{1}: \rho>\rho_{0}$, at the $95 \%$ confidence level, for selected values of $\rho_{0}$. For assessing both the intra- and inter-observer reliabilities $\rho_{\text {intra }}$ and $\rho_{\text {inter }}$ simultaneously, Eliasziw et al. (1994) suggest using the critical values $\rho_{0}=0.8$ and $\rho_{0}=0.6$, respectively, for the intra- and inter-observer reliabilities, because the former is generally recognized to be higher than the latter. These suggestions assume that one wishes to demonstrate "almost perfect" intra-observer reliability and "substantial" inter-observer reliability.

In our case, a preliminary investigation showed that the intra- and interobserver reliabilities of the $3 D$ US method were at least 0.95 and 0.85 , respectively. We therefore designed our study using Figure $2\left(\rho_{0}=0.8\right)$ of Eliasziw et al. (1994), and assuming $\rho=0.95$, for the intra-observer variability, and using Figure $1\left(\rho_{0}=0.6\right.$ ) of Eliasziw et al. (1994), and assuming $\rho=0.85$, for the inter-observer variability. On this basis, we chose a sample size of 15 
prostates, 8 observers, and 2 measurements per prostate per observer for the 3D US method, and simply used the same sample size for the HWL method.

Although we could have used fewer prostates and more measurements per prostate, this would have increased the chances of earlier results influencing subsequent measurements. Therefore, we decided to use the minimum possible number of measurements per prostate, and then use enough prostates to meet the sample size requirement.

\subsubsection{Study results}

From Table 5.5, we see that with the 3D US method, the intra- and interobserver reliabilities are $\rho_{\text {intra }}=0.99$ and $\rho_{\text {inter }}=0.96$; and with the HWL method, they are $\rho_{\text {intra }}=0.93$ and $\rho_{\text {inter }}=0.87$. Thus, the sample size used for our study happens to meet the requirements described above for both methods, and not just the 3D US method alone. Therefore, this study is well designed for investigating the intra- and inter-observer variabilities of both methods, simultaneously.

From the ANOVA results of Table 5.6, we see that the 3D US method has intra- and inter-observer errors of $S_{E M} M_{\text {inta }}=3.6 \mathrm{~cm}^{3}$ and $S E M_{\text {inter }}=9.6 \mathrm{~cm}^{3}$, respectively. By comparison, with the HWL method, these errors are $S E M_{\text {intra }}=9.5 \mathrm{~cm}^{3}$ and $S E M_{\text {inter }}=11.6 \mathrm{~cm}^{3}$, respectively. However, by doing similar analyses on different subsets of prostates, it was found that these absolute SEM values varied with the average volume of the subset, matching the earlier results of Figure 5.3 and Figure 5.4.

Thus, for each method, the volume of each prostate was normalized by its average value for that method, and the ANOVA was repeated, yielding the results shown in Table 5.7. From this table, we see that the 3D US method has respective intra- and inter-observer errors of $\mathrm{SEM}_{\text {intra }}=5.1 \%$ and $\mathrm{SEM}_{\mathrm{inter}}=$ $11.4 \%$, while the HWL method has respective intra- and inter-observer errors of SEM $_{\text {intra }}=11.5 \%$ and $\mathrm{SEM}_{\text {inter }}=13.5 \%$. 
Since SEM values are rather abstract, they are often interpreted in terms of the minimum volume change that can be confidently detected between successive measurements, which is given by $\Delta V=2.77$ SEM at the $95 \%$ level of confidence. Thus, from Table 5.7 we see that if one observer makes an initial prostate volume measurement, and the same (another) observer makes a followup measurement, then the volume must change by $32 \%(37 \%)$ before it can be confidently detected by the HWL method, but by only $14 \%$ (32\%) before it can be confidently detected by the 3D US method. This increase in sensitivity could be clinically beneficial for the diagnosis, staging, study, and management of prostate disease.

The statistical significance of the differences in the SEM (and hence $\Delta V$ ) values for the two methods were tested via the $t$-test, using the value of $t$ given by Eq. (5.13). In every case, the differences were found to be highly significant, with $p<0.001$. This demonstrates that using the 3D US method instead of the HWL method significantly increases the reliability and reduces the variability of prostate volume measurements. However, deeper insight into the difference between the performance of these two methods can be gained by considering also our earlier analyses of random and systematic observer error.

From Table 5.4, we see that, for a given method, the random errors of both radiologists and non-radiologists are almost the same. Overall, they average $3.9 \%$ for the 3D US method, and $12.6 \%$ for the HWL method. These results mirror those found for the intra-observer standard error of measurement SEM $M_{\text {intra }}$, which is a measure of the consistency and reproducibility of measurements by the same observer.

However, from Table 5.3, we see that, although the systematic errors of non-radiologists differ negligibly from those of radiologists with the 30 US method, they differ substantially with the HWL method, being much greater for non-radiologists than for radiologists. Radiologists were more consistent with the HWL method, which is familiar to them, than with the 3D US method, which 
is not. On the other hand, non-radiologists were more consistent than radiologists with the 3D US method, due perhaps to their greater familiarity with computer image technology (in this instance), but far less consistent than radiologists with the HWL method. This suggests that, although non-radiologists would need extensive training to measure prostate volumes as well as radiologists do with the HWL method, they would need little training to do so with the 3D US method. Thus, using the 3D US method, this chore could be delegated to a technician without any loss of accuracy or consistency, sparing the radiologist the tedium of performing manual planimetry, which takes one-half to two hours per prostate.

Thus, in every way, the 3D US method is superior to the HWL method for measuring prostate volume; and, unlike the HWL method, it can be performed by technicians instead of radiologists, without loss of accuracy or consistency.

\subsection{References}

1. Arcangeli CG, Ornstein DK, Keetch DW, Andriole GL. Prostate-specific antigen as a screening test for prostate cancer: The United States experience. Urol Clin North Am 24: 299-306; 1997.

2. Benson MC, Whang IS, Pantuck A, Ring K, Kaplan SA, Olsson CA, Cooner WH. Prostate specific antigen density: $A$ means of distinguishing benign prostatic hypertrophy and prostate cancer. J Urol 147:815-816; 1992.

3. Chang FM, Hsu KF, Ko HC, Yao BL, Chang CH, Yu CH, Chen HY. Threedimensional ultrasound assessment of fetal liver volume in normal pregnancy: A comparison of reproducibility with two-dimensional ultrasound and a search for a volume constant. Ultrasound Med Biol 23: 381-389; 1997.

4. Donner A, Eliasziw M. Sample size requirements for reliability studies. Stat in Med 6: 441-448; 1987. 
5. Elliot TL, Downey DB, Tong S, McLean CA, Fenster A. Accuracy of prostate volume measurements in vitro using three-dimensional ultrasound. Acad Radiol 3: 401-406; 1996.

6. Eliasziw M, Young SL, Woodbury MG, Fryday-Field K. Statistical methodology for the concurrent assessment of interrater and intrarater reliability: Using goniometric measurements as an example. Phys Ther 74: $777-788 ; 1994$.

7. Gilja $\mathrm{OH}$, Thune $\mathrm{N}$, Matre $\mathrm{K}$, Hausken $\mathrm{T}$, Odegaard $\mathrm{S}$, Berstad A. In vitro evaluation of three-dimensional ultrasonography in volume estimation of abdominal organs. Ultrasound Med Biol 20: 157-165; 1994.

8. King DL, King DL Jr, Shao MYC. Evaluation of in vitro measurement accuraccy of a three-dimensional ultrasound scanner. J Uitrasound Med 10: 77-82; 1991.

9. Mitchell JR, Karlik SJ, Lee DH, Eliasziw M, Rice GP, Fenster A. The variability of manual and computer assisted quantification of multiple sclerosis lesion volumes. Med Phys 23: 85-97; 1996a.

10. Mitchell JR, Karlik SJ, Lee DH, Eliasziw M, Rice GP, Fenster A. Quantification of multiple sclerosis lesion volumes in 1.5 and $0.5 \mathrm{~T}$ anisotropically filtered and unfiltered MR exams. Med Phys 23: 115-126; 1996b.

11. Riccabona M, Nelson TR, Pretorius DH, Davidson TE. Distance and volume measurement using three-dimensional ultrasonography. $J$ Ultrasound Med 1995; 14: 881-886.

12. Riccabona $M$, Nelson TR, Pretorius DH, Davidson TE. In vivo threedimensional sonographic measurement of organ volume: Validation in urinary bladder. J Ultrasound Med 1996; 15: 627-632.

13. Terris MK, Stamey TA. Determination of prostate volume by transrectal ultrasound. J Urol 145: 984-987, 1991.

14. Terris MK, McNeal JE, Stamey TA. Estimation of prostate cancer volume by 
transrectal ultrasound imaging. J Urol 147: 855-857, 1992.

15. Tong $S$, Downey DB, Cardinal HN, Fenster A. A three-dimensional ultrasound prostate imaging system. Ultrasound Med Biol 22: 735-746; 1996. 


\section{SUMMARY, DISCUSSION, AND FUTURE WORK}

\subsection{Summary and Discussion}

In this thesis, I have described: (i) the development of a 3D ultrasound system for imaging the prostate; (ii) the imaging performance of this system; (iii) an experimentally verified analysis of image distortion for this system; and, (iv) a study of the intra- and inter-observer variability and reliability of measuring prostate volume in vivo with 3D ultrasound, using manual planimetry, and how it compares to 2D ultrasound, using the HWL method. The following is a summary and discussion of the principal results.

A major part of the work in this thesis was to develop a 3D ultrasound system, based on a conventional $2 \mathrm{D}$ ultrasound machine and transducer. This development was successful, and was awarded a United States Patent (Fenster et al. 1996), in which I am listed as co-inventor. In the original prototype system, an IBMcompatible personal computer in the examination room was used to acquire the $2 \mathrm{D}$ image data, and a remote Sun workstation was used to reconstruct and display the resulting 3D image. After this initial development, the system was licensed to the Life Imaging Systems, Inc., of London, Ontario, and formed the basis of their Sirus ${ }^{\mathrm{TM}}$ $3 \mathrm{D}$ ultrasound imaging system. In the commercial version of this system, a single Macintosh computer is used to carry out both of these tasks. However, the principle remains the same.

In our system, a side-firing probe, axially rotated under computer control, is used to acquire the $2 D$ images from which the $3 D$ image is reconstructed. Therefore, for an undistorted reconstruction, the inner radius $R_{0}$ of the $2 D$ images and the total scanning angle $\Theta$ must be known accurately. Theoretically, the relative distortions in the shape, length, area, and volume of an object, due to an error $\Delta R$ in $R_{0}$ or $\Delta \Theta$ in $\Theta$, all vary as $P \Delta R / R+Q \Delta \Theta / \Theta$, where $|P| \leq 1,|Q| \leq 1$, and $R$ is the average distance of the object from the axis. In every case, the simple theoretical formulas for $P$ and $Q$ agreed with distortion measurements from both simulated and experimental images to within the 
measurement uncertainty. Thus, all four types of distortion will be negligible if $\Delta R / R \ll 1$ and $\Delta \Theta / \Theta \ll 1$. We have described simple calibration procedures for achieving this, and determining the image scale, as well. For a wellcalibrated system, where $|\Delta R / R|+|\Delta \Theta / \Theta|<0.01$, all four types of image distortion will thus be less than $1 \%$. Moreover, image distances, areas, and volumes will in general all have similar accuracy.

In addition to imaging the prostate, which has been the focus of this thesis, this system has also been adapted to imaging the eye and orbit (Downey et al. 1995b, 1995c, 1996), the breast (Downey and Fenster 1995, Fenster et al. 1995), the kidney (Downey and Fenster 1995), the vasculature (Downey and Fenster 1995), and the heart (Boughner et al. 1995), and to performing 3D ultrasoundguided cryosurgery (Downey et al. 1995a, Chin et al. 1996). Potential applications of this system include 3D ultrasound imaging of the fetus or the liver, and 3D ultrasound-guided biopsy or brachytherapy. Thus, this system has broad utility and many clinical applications.

Used as a 3D TRUS system for imaging the prostate, our 3D ultrasound imaging system offers the following advantages in comparison to a conventional $2 \mathrm{D}$ TRUS system: patient examination time is reduced from 10-20 minutes to 2-5 minutes, benefiting both patients and clinicians; prostate images can be viewed interactively in multiple simultaneous planes, allowing better visualization of the organ's internal architecture; the same viewing plane can be easily retrieved in successive examinations, making 3D ultrasound imaging well-suited to performing prospective or follow-up studies; and, more accurate and consistent volume measurements can be made, allowing better monitoring of the response of the organ or tumour to therapy.

The accuracy and precision of distance and volume measurements made with the system were evaluated using laboratory test phantoms. These results showed that distance measurements have $1.2 \%$ accuracy and $2.9 \%$ precision, while volume measurements have $0.9 \%$ accuracy and $1.7 \%$ precision. 
The accuracy and precision of volume measurements of actual prostates was then evaluated in vitro using specimens harvested from cadavers. By comparison with volumes measured by water displacement, it was found that the prostate volumes measured by manual planimetry (the 3D US method) had an accuracy of $2.6 \%$ and a precision of $2.5 \%$.

In the above performance evaluation, the results were based on scans of objects in a water tank, whose image boundaries were clearly defined, so that operator errors were small. However, for images scanned in vivo, the boundary of the prostate gland is not well-defined, which can lead to both intra- and interoperator variability in prostate volume measurements. Therefore, in order to assess the accuracy and consistency of prostate volume measurements in vivo, a clinical study of the intra- and inter-observer variability and reliability was conducted, using both the HWL method (with 2D US images) and manual planimetry (the 3D US method).

In this study, eight observers were recruited to perform two independent volume measurements for each method on each of fifteen 3D prostate images. An analysis of variance (ANOVA) of these measurements then showed that the intra- and inter-observer standard errors SEMintra and SEMimer were respectively $5.1 \%$ and $11.4 \%$ for the 3D US method, and $11.5 \%$ and $13.5 \%$ for the HWL method, while the intra- and inter-observer reliabilities $\rho_{i n t r a}$ and $\rho_{\text {inner }}$ were respectively 0.99 and 0.96 for the 3D US method, and 0.93 and 0.87 for the HWL method. Thus, the 3D US method is superior to the HWL method for measuring prostate volumes.

A further advantage of the 3D US method over the HWL method is that, with the former, there was statistically no significant difference in performance between radiologists and non-radiologists (technicians and graduate students). Hence, using the 30 US method, a technician could relieve the radiologist from the tedium of performing manual planimetry, which requires from one-half to two hours per prostate, without any loss in accuracy or consistency in the results. 
The significance of these results is that the accuracy and consistency of clinical prostate volume estimates can be greatly increased by switching from the HWL method to the 3D US method, thereby improving the accuracy and consistency of the staging of prostate cancer and the interpretation of PSA levels in the monitoring of prostate cancer or BPH. This improvement, together with the enhanced visualization of the prostate and its surrounding tissues afforded by $3 \mathrm{D}$ imaging, e.g. via multi-planar texture mapping, may result in higher diagnostic accuracy and consistency, and hence better management of prostate disease, which is the overall goal of this work.

\subsection{Future Work}

\subsubsection{Improvement of the system}

The 3D ultrasound imaging system described here is an add-on system, i.e. it is a microcomputer-based system added on to an existing conventional $2 D$ ultrasound machine, which digitizes the analog video signals from the machine's video output port, stores them in memory, and reconstructs them into a $3 D$ ultrasound image.

In our system, we chose to use a frame-grabber to digitize the video signals on-the-fly, directly from the port, rather than from a videotape recording of them, in order to avoid any signal degradation by the videotaping process. However, since the 2D image data from the transducer is already in digital format, and converted to analog format before being output by the ultrasound machine, an improved approach would be to use this data directly, before conversion, avoiding the need to reconvert the data back to digital form in the microcomputer. This would eliminate two data conversions: one digital-to-analog and one analog-to-digital.

An even better approach would be to integrate the 3D add-on system directly into the 2D ultrasound machine to form a single 3D ultrasound machine.

However, adopting either one of these approaches would require design modifications to be made to the ultrasound machines by their manufacturers. 


\subsubsection{Effect of 2D image slice thickness}

A 2D ultrasound image represents a cross-section of the human anatomy of finite thickness. Because of this, image artifacts are produced by off-center echoes being displayed in the $2 \mathrm{D}$ image as if they were coming from the middle of the imaging plane. This artifact is more easily seen in 30 ultrasound images. The reason for this is as follows:

Since the 3D image data set is composed of a series of 2D images taken while the ultrasound transducer moves perpendicularly to the $2 \mathrm{D}$ image planes, the reconstructed 3D image of an object can be described as the convolution of the original object with the profile of the ultrasound beam, across the $2 D$ slice thickness. Thus, the 3D image of a sphere will be slightly ellipsoidal, the artifact being smallest near the focal zone, where the ultrasound beam is narrowest, and worsening further away from the transducer, as the beam diverges.

Theoretically, if the beam profile were known, a deconvolution algorithm could be used to correct this artifact, for an ideal image. However, since real ultrasound images contain both noise and speckle, deconvolution is difficult. More research is needed in this area.

\subsubsection{Automated image segmentation}

Although prostate volume estimation via the 3D US method, i.e. manual planimetry, has been shown to be more accurate and precise than via the conventional HWL method, it is tedious and time-consuming to perform, requiring anywhere from one-half to two hours for a single prostate, depending on its size and the slice spacing used.

One solution to this problem would be to invent a computer algorithm to perform automated image segmentation. A semi-automated segmentation of prostate based on the "deformable active contours" model was briefly examined for prostate tumours, since they are anechoic and therefore have a well-defined image boundary. In this model, the operator outlines the tumour in three slices: 
the first slice, a middle slice, and the final slice. Using these as a guide, the algorithm then deforms an active contour, from slice-to-slice, in order to outline the tumour in the other slices, by minimizing an energy function. Two types of energy are considered: internal energy, due to contour elasticity and rigidity; and external energy, due to the ultrasound image. However, although preliminary tests of this algorithm on phantom images show encouraging results, they also show that it needs to be made more robust before it can be reliably used for ultrasound image segmentation.

At the last annual meeting of the Canadian Organization of Medical Physicists (COMP), Sinasac and Peters (1997) reported on the use of a 3D deformable surface model for the intraoperative visualization and quantification of cerebral tissue resection. This model can fit closed or open surfaces to volumetric data. The surface is first discretized using finite elements, and the model is then fitted to it by minimizing an energy function, which describes the deformation energy of the surface. This technique may be applicable to the automated segmentation of 3D ultrasound prostate images.

\subsection{References}

1. Boughner DR, Pfugfelder PW, Dietrich J, Fenster A. Three-dimensional color Doppler echocardiography: Clinical applications. Submitted to Amer Soc of Echocard.

2. Chin $\mathrm{JL}$, Downey $D B$, Onik G, Fenster $A$. Three-dimensional prostate ultrasound and its application to cryosurgery. Techniques in Urology 2: 187-193; 1996.

3. Downey DB, Chin JL, Fenster A. Three-dimensional US-guided cryosurgery. Radiology 197(P): 539; 1995 .

4. Downey DB, Fenster A. Vascular imaging with a three-dimensional power Doppler system. AJR 165: 665-668; 1995. 
5. Downey DB, Nicolle DA, Fenster A. Three-dimensional orbital ultrasonography. Can J Opthamol 30: 395-398; 1995 b.

6. Downey DB, Nicolle DA, Fenster A. Three-dimensional ultrasound of the eye. Administrative Radiology Journal 14:46-50; $1995 \mathrm{c}$.

7. Downey DB, Nicolle DA, Levin MF, Fenster A. Three-dimensional ultrasound imaging of the eye. Eye 10:75-81; 1996.

8. Fenster A, Tong S, Sherebrin S, Downey DB, Rankin RN. Three-dimensional ultrasound imaging. Medical Imaging 1995: Physics of Medical Imaging, Proc SPIE 2432: 176-184, 1995.

9. Sinasac MJ, Peters TM. Use of 3D deformable surface model for intraoperative visualization and quantification of cerebral tissue resection. Proceedings of the 43rd annual meeting of the Canadian Organization of Medical Physicists, July 9 - 12, Charlottetown, PEI: p212-214. 
TEST TARGET (QA-3)
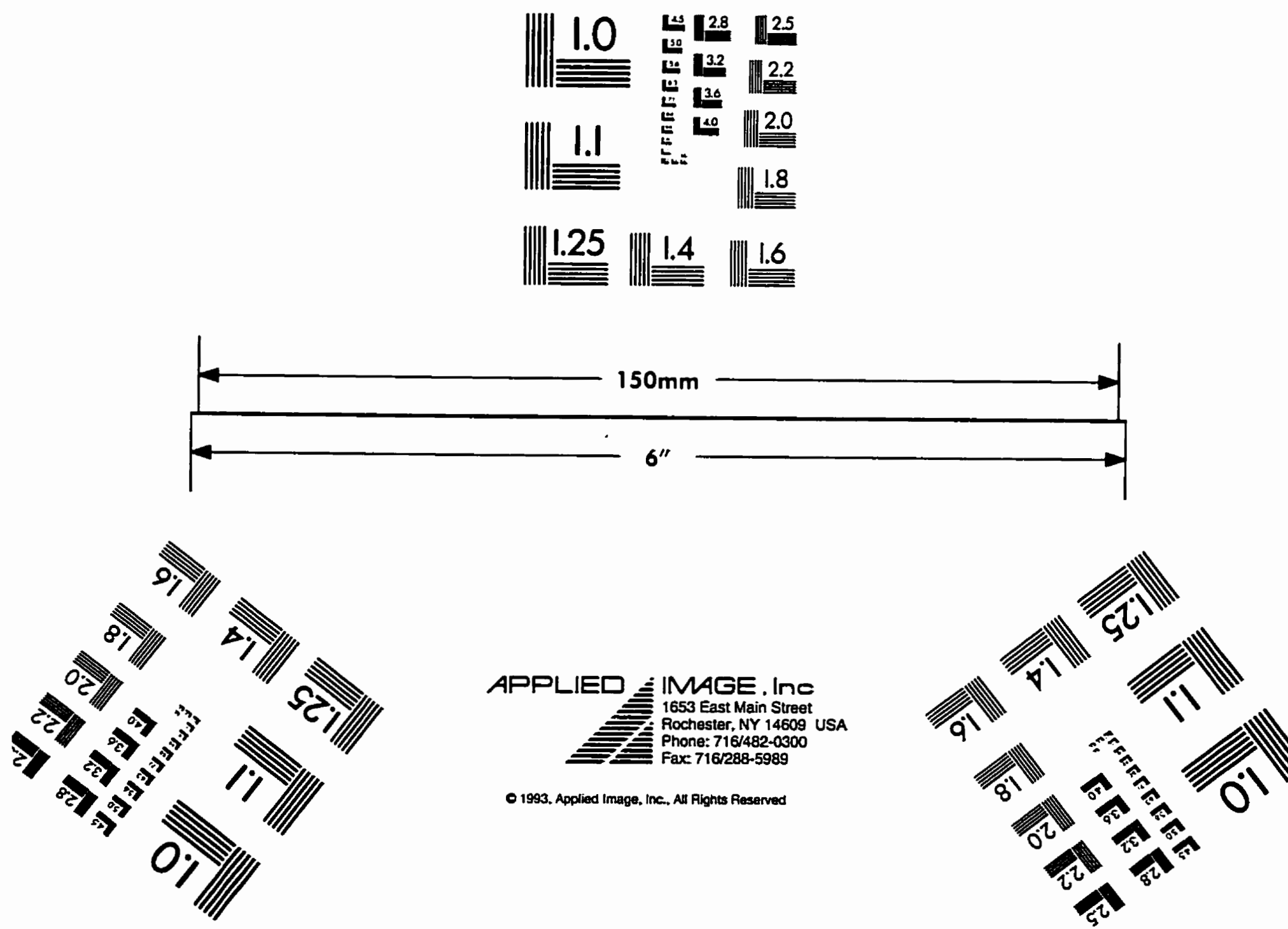